\title{
MANIFOLDS OF MAPPINGS FOR CONTINUUM MECHANICS
}

\author{
PETER W. MICHOR
}

\begin{abstract}
After an introduction to convenient calculus in infinite dimensions, the foundational material for manifolds of mappings is presented. The central character is the smooth convenient manifold $C^{\infty}(M, N)$ of all smooth mappings from a finite dimensional Whitney manifold germ $M$ into a smooth manifold $N$. A Whitney manifold germ is a smooth (in the interior) manifold with a very general boundary, but still admitting a continuous Whitney extension operator. This notion is developed here for the needs of geometric continuum mechanics.
\end{abstract}

2010 Mathematics Subject Classification: 58B20, 58D15, 35Q31

Keywords and Phrases: Convenient calculus, Manifolds of mappings, Diffeomorphism groups, Shape spaces, weak Riemannian metrics.

\section{Contents}

1. Introduction 2

2. A short review of convenient calculus in infinite dimensions 3

3. Manifolds with corners 8

4. Whitney manifold germs 12

5. Manifolds of mappings 20

6. Regular Lie groups 28

7. Spaces of embeddings or immersions, and shape spaces

8. Weak Riemannian manifolds 47

9. Robust weak Riemannian manifolds and Riemannian submersions 56

References 62

Peter W. Michor: Universität Wien, Oskar-Morgenstern-Platz 1, A-1090 Wien, Austria. 


\section{INTRODUCTION}

At the birthplace of the notion of manifolds, in the Habilitationsschrift [93, end of section I], Riemann mentioned infinite dimensional manifolds explicitly. The translation into English in [94] reads as follows:

There are manifoldnesses in which the determination of position requires not a finite number, but either an endless series or a continuous manifoldness of determinations of quantity. Such manifoldnesses are, for example, the possible determinations of a function for a given region, the possible shapes of a solid figure, $8 c$.

Reading this with a lot of good will one can interpret it as follows: When Riemann sketched the general notion of a manifold, he also had in mind the notion of an infinite dimensional manifold of mappings between manifolds. He then went on to describe the notion of Riemannian metric and to talk about curvature.

The dramatis personae of this foundational chapter are named in the following diagram:

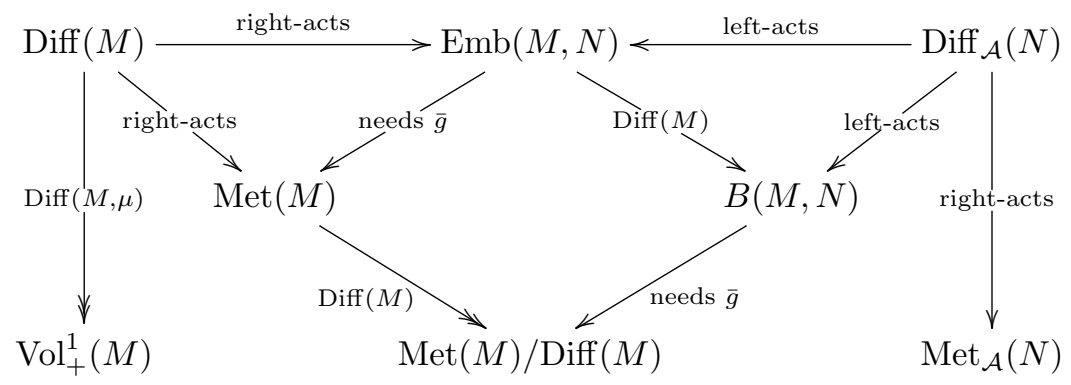

In this diagram:

$M$ is a finite dimensional compact smooth manifold.

$N$ is a finite dimensional smooth manifolds without boundary, and $\bar{g}$ is one fixed background Riemannian metric on $N$ which we always assume to be of bounded geometry; see Section 5 .

$\operatorname{Met}(N)=\Gamma\left(S_{+}^{2} T^{*} N\right)$ is the space of all Riemannian metrics on $N$.

$\operatorname{Diff}(M)$ is the regular Fréchet Lie group of all diffeomorphisms on the compact manifold $M$ with corners.

$\operatorname{Diff}_{\mathcal{A}}(N), \mathcal{A} \in\left\{H^{\infty}, \mathcal{S}, c\right\}$ the regular Lie group of all smooth diffeomorphisms of decay $\mathcal{A}$ towards $\operatorname{Id}_{N}$.

$\operatorname{Emb}(M, N)$ is the infinite dimensional smooth manifold of all embeddings $M \rightarrow N$, which is the total space of a smooth principal fiber bundle $\operatorname{Emb}(M, N) \rightarrow B(M, N)=\operatorname{Imm}(M, N) / \operatorname{Diff}(M)$ with structure group $\operatorname{Diff}(M)$ and base manifold $B(M, N)$, the space of all smooth submanifolds of $N$ of type $M$. It is possible to extend $\operatorname{Emb}(M, N)$ to the manifold of $\operatorname{Imm}(M, N)$ and $B(M, N)$ to the infinite dimensional orbifold $B_{i}(M, N)$. 
$\operatorname{Vol}_{+}^{1}(M) \subset \Gamma(\operatorname{vol}(M))$ is the space of all positive smooth probability densities on the manifold $M$ with corners.

Since it will be of importance for geometric continuum mechanics, I will allow the source manifold $M$ to be quite general: $M$ can be a manifold with corners; see Section 3. This setting is worked out in detail in 69. Or $M$ can be a Whitney manifold germ, a notion originating in this paper; see Sec. 4 .

In this foundational chapter I will describe the theory of manifolds of mappings, of groups of diffeomorphisms, of manifolds of submanifolds (with corners), and of some striking results about weak Riemannian geometry on these spaces. See [10] for an overview article which is much more comprehensive for the aspect of shape spaces.

An explicit construction of manifolds of smooth mappings modeled on Fréchet spaces was described by [28]. Differential calculus beyond the realm of Banach spaces has some inherent difficulties even in its definition; see section 2. Smoothness of composition and inversion was first treated on the group of all smooth diffeomorphisms of a compact manifold in 63]; however, there was a gap in the proof, which was first filled by [48. Manifolds of $C^{k}$-mappings and/or mappings of Sobolev classes were treated by [31, 27, Smale-Abraham [1] and 92]. Since these are modeled on Banach spaces, they allow solution methods for equations and have found a lot of applications. See in particular [26].

In preparation of this chapter I noticed, that the canonical chart construction for the manifold $C^{\infty}(M, N)$ even works if we allow $M$ to be a Whitney manifold germ: These are modeled on open subsets of closed subsets of $\mathbb{R}^{m}$ which (1) admit a continuous Whitney extension operator and (2) are the closure of their interior. See Section 4 for a thorough discussion. Many results for them described below are preliminary, e.g., 6.4 7.2. I expect that they can be strengthened considerably, but I had not enough time to pursue them during the preparation of this chapter.

I thank Reuven Segev and Marcelo Epstein for asking me for a contribution to this volume, and I thank them and Leonhard Frerick, Andreas Kriegl, Jochen Wengenroth, and Armin Rainer for helpful discussions.

\section{A SHORT REVIEW OF CONVENIENT CALCULUS IN INFINITE DIMENSIONS}

Traditional differential calculus works well for finite dimensional vector spaces and for Banach spaces. Beyond Banach spaces, the main difficulty is that composition of linear mappings stops to be jointly continuous at the level of Banach spaces, for any compatible topology. Namely, if for a locally convex vector space $E$ and its dual $E^{\prime}$ the evaluation mapping ev $: E \times E^{\prime} \rightarrow \mathbb{R}$ is jointly continuous, then there are open neighborhoods of zero $U \subset E$ and $U^{\prime} \subset E^{\prime}$ with $\operatorname{ev}\left(U \times U^{\prime}\right) \subset[-1,1]$. But then $U^{\prime}$ is contained in the polar of the open set $U$, and thus is bounded. So $E^{\prime}$ is normable, and a fortiori $E$ is normable.

For locally convex spaces which are more general than Banach spaces, we sketch here the convenient approach as explained in [44] and [55]. 
The name convenient calculus mimics the paper 98 whose results (but not the name 'convenient') was predated by [17, [18, [19]. They discussed compactly generated spaces as a cartesian closed category for algebraic topology. Historical remarks on only those developments of calculus beyond Banach spaces that led to convenient calculus are given in [55, end of chapter I, p. $73 \mathrm{ff}]$.

2.1. The $c^{\infty}$-topology. Let $E$ be a locally convex vector space. A curve $c: \mathbb{R} \rightarrow E$ is called smooth or $C^{\infty}$ if all derivatives exist and are continuous. Let $C^{\infty}(\mathbb{R}, E)$ be the space of smooth curves. It can be shown that the set $C^{\infty}(\mathbb{R}, E)$ does not entirely depend on the locally convex topology of $E$, only on its associated bornology (system of bounded sets); see [55, 2.11]. The final topologies with respect to the following sets of mappings into $E$ (i.e., the finest topology on $E$ such that each map is continuous) coincide; see [55, 2.13]:

(1) $C^{\infty}(\mathbb{R}, E)$.

(2) The set of all Lipschitz curves (so that $\left\{\frac{c(t)-c(s)}{t-s}: t \neq s,|t|,|s| \leq C\right\}$ is bounded in $E$, for each $C$ ).

(3) The set of injections $E_{B} \rightarrow E$ where $B$ runs through all bounded absolutely convex subsets in $E$, and where $E_{B}$ is the linear span of $B$ equipped with the Minkowski functional $\|x\|_{B}:=\inf \{\lambda>0: x \in$ $\lambda B\}$.

(4) The set of all Mackey-convergent sequences $x_{n} \rightarrow x$ (i.e., those for which there exists a sequence $0<\lambda_{n} \nearrow \infty$ with $\lambda_{n}\left(x_{n}-x\right)$ bounded).

The resulting unique topology is called the $c^{\infty}$-topology on $E$ and we write $c^{\infty} E$ for the resulting topological space.

In general (on the space $\mathcal{D}$ of test functions for example) it is finer than the given locally convex topology, it is not a vector space topology, since addition is no longer jointly continuous. Namely, even $c^{\infty}(\mathcal{D} \times \mathcal{D}) \neq c^{\infty} \mathcal{D} \times c^{\infty} \mathcal{D}$.

The finest among all locally convex topologies on $E$ which are coarser than $c^{\infty} E$ is the bornologification of the given locally convex topology. If $E$ is a Fréchet space, then $c^{\infty} E=E$.

2.2. Convenient vector spaces. A locally convex vector space $E$ is said to be a convenient vector space if one of the following equivalent conditions holds (called $c^{\infty}$-completeness); see [55, 2.14]:

(1) For any $c \in C^{\infty}(\mathbb{R}, E)$ the (Riemann-) integral $\int_{0}^{1} c(t) d t$ exists in $E$.

(2) Any Lipschitz curve in $E$ is locally Riemann integrable.

(3) A curve $c: \mathbb{R} \rightarrow E$ is $C^{\infty}$ if and only if $\lambda \circ c$ is $C^{\infty}$ for all $\lambda \in E^{*}$, where $E^{*}$ is the dual of all continuous linear functionals on $E$.

- Equivalently, for all $\lambda \in E^{\prime}$, the dual of all bounded linear functionals.

- Equivalently, for all $\lambda \in \mathcal{V}$, where $\mathcal{V}$ is a subset of $E^{\prime}$ which recognizes bounded subsets in $E$; see [55, 5.22]

We call this scalarwise $C^{\infty}$. 
(4) Any Mackey-Cauchy sequence (i.e., $t_{n m}\left(x_{n}-x_{m}\right) \rightarrow 0$ for some $t_{n m} \rightarrow \infty$ in $\left.\mathbb{R}\right)$ converges in $E$. This is visibly a mild completeness requirement.

(5) If $B$ is bounded closed absolutely convex, then $E_{B}$ is a Banach space.

(6) If $f: \mathbb{R} \rightarrow E$ is scalarwise $\operatorname{Lip}^{k}$, then $f$ is $\operatorname{Lip}^{k}$, for $k>1$.

(7) If $f: \mathbb{R} \rightarrow E$ is scalarwise $C^{\infty}$ then $f$ is differentiable at 0 .

Here a mapping $f: \mathbb{R} \rightarrow E$ is called Lip ${ }^{k}$ if all derivatives up to order $k$ exist and are Lipschitz, locally on $\mathbb{R}$. That $f$ is scalarwise $C^{\infty}$ (resp., Lip ${ }^{k}$ ) means $\lambda \circ f$ is $C^{\infty}$ (resp., Lip ${ }^{k}$ ) for all continuous (equiv., bounded) linear functionals on $E$.

2.3. Smooth mappings. Let $E$, and $F$ be convenient vector spaces, and let $U \subset E$ be $c^{\infty}$-open. A mapping $f: U \rightarrow F$ is called smooth or $C^{\infty}$, if $f \circ c \in C^{\infty}(\mathbb{R}, F)$ for all $c \in C^{\infty}(\mathbb{R}, U)$. See [55, 3.11].

If $E$ is a Fréchet space, then this notion coincides with all other reasonable notions of $C^{\infty}$-mappings; see below. Beyond Fréchet spaces, as a rule, there are more smooth mappings in the convenient setting than in other settings, e.g., $C_{c}^{\infty}$. Moreover, any smooth mapping is continuous for the $c^{\infty}$-topologies, but in general not for the locally convex topologies: As shown in the beginning of Section 2, the evaluation mapping ev : $E \times E^{\prime} \rightarrow \mathbb{R}$ is continuous only if $E$ is normable. On Fréchet spaces each smooth mapping is continuous; see the end of 2.1

2.4. Main properties of smooth calculus. In the following all locally convex spaces are assumed to be convenient.

(1) For maps on Fréchet spaces the notion of smooth mapping from 2.3 coincides with all other reasonable definitions. On $\mathbb{R}^{2}$ this is a nontrivial statement; see [16] or [55, 3.4].

(2) Multilinear mappings are smooth if and only if they are bounded; see [55, 5.5].

(3) If $E \supseteq U \stackrel{f}{\longrightarrow} F$ is smooth then the derivative $d f: U \times E \rightarrow F$ is smooth, and also $d f: U \rightarrow L(E, F)$ is smooth where $L(E, F)$ denotes the convenient space of all bounded linear mappings with the topology of uniform convergence on bounded subsets; see 55 , $3.18]$.

(4) The chain rule holds; see [55, 3.18].

(5) The space $C^{\infty}(U, F)$ is again a convenient vector space where the structure is given by the injection

$$
C^{\infty}(U, F) \stackrel{C^{\infty}(c, \lambda)}{\longrightarrow} \prod_{c \in C^{\infty}} C_{(\mathbb{R}, U), \lambda \in F^{*}}^{\infty}(\mathbb{R}, \mathbb{R}), \quad f \mapsto(\lambda \circ f \circ c)_{c, \lambda},
$$

and where $C^{\infty}(\mathbb{R}, \mathbb{R})$ carries the topology of compact convergence in each derivative separately; see [55, 3.11 and 3.7].

(6) The exponential law holds; see [55, 3.12].: For $c^{\infty}$-open $V \subset F$,

$$
C^{\infty}\left(U, C^{\infty}(V, G)\right) \cong C^{\infty}(U \times V, G)
$$


is a linear diffeomorphism of convenient vector spaces.

Note that this result (for $U=\mathbb{R}$ ) is the main assumption of variational calculus. Here it is a theorem.

(7) A linear mapping $f: E \rightarrow C^{\infty}(V, G)$ is smooth (by (2) equivalent to bounded) if and only if $E \stackrel{f}{\longrightarrow} C^{\infty}(V, G) \stackrel{\mathrm{ev}_{v}}{\longrightarrow} G$ is smooth for each $v \in V$. (Smooth uniform boundedness theorem; see [55, theorem 5.26].)

(8) A mapping $f: U \rightarrow L(F, G)$ is smooth if and only if

$$
U \stackrel{f}{\longrightarrow} L(F, G) \stackrel{\mathrm{ev}_{v}}{\longrightarrow} G
$$

is smooth for each $v \in F$, because then it is scalarwise smooth by the classical uniform boundedness theorem.

(9) The following canonical mappings are smooth. This follows from the exponential law by simple categorical reasoning; see [55, 3.13].

$$
\begin{aligned}
& \text { ev }: C^{\infty}(E, F) \times E \rightarrow F, \quad \operatorname{ev}(f, x)=f(x) \\
& \text { ins }: E \rightarrow C^{\infty}(F, E \times F), \quad \operatorname{ins}(x)(y)=(x, y) \\
& (\quad)^{\wedge}: C^{\infty}\left(E, C^{\infty}(F, G)\right) \rightarrow C^{\infty}(E \times F, G) \\
& (\quad)^{\vee}: C^{\infty}(E \times F, G) \rightarrow C^{\infty}\left(E, C^{\infty}(F, G)\right) \\
& \operatorname{comp}: C^{\infty}(F, G) \times C^{\infty}(E, F) \rightarrow C^{\infty}(E, G) \\
& C^{\infty}(, \quad): C^{\infty}\left(F, F_{1}\right) \times C^{\infty}\left(E_{1}, E\right) \rightarrow \\
& \quad \rightarrow C^{\infty}\left(C^{\infty}(E, F), C^{\infty}\left(E_{1}, F_{1}\right)\right) \\
& \quad(f, g) \mapsto(h \mapsto f \circ h \circ g) \\
& \prod: \prod C^{\infty}\left(E_{i}, F_{i}\right) \rightarrow C^{\infty}\left(\prod E_{i}, \prod F_{i}\right)
\end{aligned}
$$

This ends our review of the standard results of convenient calculus. Just for the curious reader and to give a flavor of the arguments, we enclose a lemma that is used many times in the proofs of the results above.

Lemma. (Special curve lemma, [55, 2.8]) Let $E$ be a locally convex vector space. Let $x_{n}$ be a sequence which converges fast to $x$ in E; i.e., for each $k \in \mathbb{N}$ the sequence $n^{k}\left(x_{n}-x\right)$ is bounded. Then the infinite polygon through the $x_{n}$ can be parameterized as a smooth curve $c: \mathbb{R} \rightarrow E$ such that $c\left(\frac{1}{n}\right)=x_{n}$ and $c(0)=x$.

2.5. Remark. Convenient calculus (i.e., having properties 6 and 7) exists also for:

- Real analytic mappings; see [54] or [55, Chapter II].

- Holomorphic mappings; see [62] or [55, Chapter II] (using the notion of [35, 36]).

- Many classes of Denjoy-Carleman ultradifferentiable functions, both of Beurling type and of Roumieu type, see [57, 58, 59, 61.

- With some adaptations, $\operatorname{Lip}^{k}$; see [44. One has to adapt the exponential law 2.4.9 in the obvious way. 
- With more adaptations, even $C^{k, \alpha}$ (the $k$-th derivative is Höldercontinuous with index $0<\alpha \leq 1$ ); see [38], 37]. Namely, if $f$ is $C^{k, \alpha}$ and $g$ is $C^{k, \beta}$, then $f \circ g$ is $C^{k, \alpha \beta}$.

Differentiability $C^{n}$ cannot be described by a convenient approach (i.e., allowing result like 2.4). Only such differentiability notions allow this, which can be described by boundedness conditions only.

We shall treat $C^{n}$ mapping spaces using the following result.

2.6. Recognizing smooth curves. The following result is very useful if one wants to apply convenient calculus to spaces which are not tied to its categorical origin, like the Schwartz spaces $\mathcal{S}, \mathcal{D}$, or Sobolev spaces; for its uses see [77] and [60]. In what follows $\sigma(E, \mathcal{V})$ denotes the initial (also called weak) topology on $E$ with respect to a set $\mathcal{V} \subset E^{\prime}$.

Theorem. [44, Theorem 4.1.19] Let $c: \mathbb{R} \rightarrow E$ be a curve in a convenient vector space $E$. Let $\mathcal{V} \subset E^{\prime}$ be a subset of bounded linear functionals such that the bornology of $E$ has a basis of $\sigma(E, \mathcal{V})$-closed sets. Then the following are equivalent:

(1) c is smooth

(2) There exist locally bounded curves $c^{k}: \mathbb{R} \rightarrow E$ such that $\lambda \circ c$ is smooth $\mathbb{R} \rightarrow \mathbb{R}$ with $(\lambda \circ c)^{(k)}=\lambda \circ c^{k}$, for each $\lambda \in \mathcal{V}$.

If $E=F^{\prime}$ is the dual of a convenient vector space $F$, then for any point separating subset $\mathcal{V} \subseteq F$ the bornology of $E$ has a basis of $\sigma(E, \mathcal{V})$-closed subsets, by [44, 4.1.22].

This theorem is surprisingly strong: note that $\mathcal{V}$ does not need to recognize bounded sets. We shall use the theorem in situations where $\mathcal{V}$ is just the set of all point evaluations on suitable Sobolev spaces.

2.7. Frölicher spaces. Following [55, Section 23] we describe here the following simple concept. A Frölicher space or a space with smooth structure is a triple $\left(X, \mathcal{C}_{X}, \mathcal{F}_{X}\right)$ consisting of a set $X$, a subset $\mathcal{C}_{X}$ of the set of all mappings $\mathbb{R} \rightarrow X$, and a subset $\mathcal{F}_{X}$ of the set of all functions $X \rightarrow \mathbb{R}$, with the following two properties:

(1) A function $f: X \rightarrow \mathbb{R}$ belongs to $\mathcal{F}_{X}$ if and only if $f \circ c \in C^{\infty}(\mathbb{R}, \mathbb{R})$ for all $c \in \mathcal{C}_{X}$.

(2) A curve $c: \mathbb{R} \rightarrow X$ belongs to $\mathcal{C}_{X}$ if and only if $f \circ c \in C^{\infty}(\mathbb{R}, \mathbb{R})$ for all $f \in \mathcal{F}_{X}$.

Note that a set $X$ together with any subset $\mathcal{F}$ of the set of functions $X \rightarrow \mathbb{R}$ generates a unique Frölicher space $\left(X, \mathcal{C}_{X}, \mathcal{F}_{X}\right)$, where we put in turn:

$$
\begin{aligned}
& \mathcal{C}_{X}:=\left\{c: \mathbb{R} \rightarrow X: f \circ c \in C^{\infty}(\mathbb{R}, \mathbb{R}) \text { for all } f \in \mathcal{F}\right\}, \\
& \mathcal{F}_{X}:=\left\{f: X \rightarrow \mathbb{R}: f \circ c \in C^{\infty}(\mathbb{R}, \mathbb{R}) \text { for all } c \in \mathcal{C}_{X}\right\},
\end{aligned}
$$

so that $\mathcal{F} \subseteq \mathcal{F}_{X}$. The set $\mathcal{F}$ will be called a generating set of functions for the Frölicher space. Similarly a set $X$ together with any subset $\mathcal{C}$ of the set of curves $\mathbb{R} \rightarrow X$ generates a Frölicher space; $\mathcal{C}$ is then called a generating 
set of curves for this Frölicher structure. Note that a locally convex space $E$ is convenient if and only if it is a Frölicher space with the structure whose space $\mathcal{C}_{E}$ of smooth curves is the one described in 2.1, or whose space $\mathcal{F}_{E}$ of smooth functions is described in 2.3 . This follows directly from 2.2 .

On each Frölicher space we shall consider the final topology with respect to all smooth curves $c: \mathbb{R} \rightarrow X$ in $\mathcal{C}_{X}$; i.e., the coarsest topology such that each such $c$ is continuous. This is in general finer that the initial topology with respect to all functions in $\mathcal{F}_{X}$.

A mapping $\varphi: X \rightarrow Y$ between two Frölicher spaces is called smooth if one of the following three equivalent conditions hold

(3) For each $c \in \mathcal{C}_{X}$ the composite $\varphi \circ c$ is in $\mathcal{C}_{Y}$. Note that here $\mathcal{C}_{X}$ can be replaced by a generating set $\mathcal{C}$ of curves in $X$.

(4) For each $f \in \mathcal{F}_{Y}$ the composite $f \circ \varphi$ is in $\mathcal{F}_{X}$. Note that $\mathcal{F}_{Y}$ can be replaced by a generating set of functions.

(5) For each $c \in \mathcal{C}_{X}$ and for each $f \in \mathcal{F}_{Y}$ the composite $f \circ \varphi \circ c$ is in $C^{\infty}(\mathbb{R}, \mathbb{R})$.

The set of all smooth mappings from $X$ to $Y$ will be denoted by $C^{\infty}(X, Y)$. Then we have $C^{\infty}(\mathbb{R}, X)=\mathcal{C}_{X}$ and $C^{\infty}(X, \mathbb{R})=\mathcal{F}_{X}$.

Frölicher spaces and smooth mappings form a category which is complete, cocomplete, and cartesian closed, by [55, 23.2].

Note that there is the finer notion of diffeological spaces $X$ introduced by Souriau: These come equipped with a set of mappings from open subsets of $\mathbb{R}^{n}$ 's into $X$ subject to some obvious properties concerning reparameterizations by $C^{\infty}$-mappings; see [51]. The obvious functor associating the generated Frölicher space to a diffeological space is both left and right adjoint to the embedding of the category of Frölicher spaces into the category of diffeological spaces. A characterization of those diffeological spaces which are Frölicher spaces is in [106, Section 2.3].

\section{MANifOLDS With CORNERS}

In this section we collect some results which are essential for the extension of the convenient setting for manifolds of mappings to a source manifold which has corners and which need not be compact.

3.1. Manifolds with corners. For more information we refer to [25, 69, [66]. Let $Q=Q^{m}=\mathbb{R}_{\geq 0}^{m}$ be the positive orthant or quadrant. By Whitney's extension theorem or Seeley's theorem (see also the discussion in 4.1-4.3), the restriction $C^{\infty}\left(\mathbb{R}^{m}\right) \rightarrow C^{\infty}(Q)$ is a surjective continuous linear mapping which admits a continuous linear section (extension mapping); so $C^{\infty}(Q)$ is a direct summand in $C^{\infty}\left(\mathbb{R}^{m}\right)$. A point $x \in Q$ is called a corner of codimension (or index) $q>0$ if $x$ lies in the intersection of $q$ distinct coordinate hyperplanes. Let $\partial^{q} Q$ denote the set of all corners of codimension $q$.

A manifold with corners (recently also called a quadrantic manifold) $M$ is a smooth manifold modeled on open subsets of $Q^{m}$. We assume that it is 
connected and second countable; then it is paracompact and each open cover admits a subordinated smooth partition of unity.

We do not assume that $M$ is oriented, but for Moser's theorem we will eventually assume that $M$ is compact. Let $\partial^{q} M$ denote the set of all corners of codimension $q$. Then $\partial^{q} M$ is a submanifold without boundary of codimension $q$ in $M$; it has finitely many connected components if $M$ is compact. We shall consider $\partial M$ as stratified into the connected components of all $\partial^{q} M$ for $q>0$. Abusing notation we will call $\partial^{q} M$ the boundary stratum of codimension $q$; this will lead to no confusion. Note that $\partial M$ itself is not a manifold with corners. We shall denote by $j_{\partial^{q} M}: \partial^{q} M \rightarrow M$ the embedding of the boundary stratum of codimension $q$ into $M$, and by $j_{\partial M}: \partial M \rightarrow M$ the whole complex of embeddings of all strata.

Each diffeomorphism of $M$ restricts to a diffeomorphism of $\partial M$ and to a diffeomorphism of each stratum $\partial^{q} M$. The Lie algebra of $\operatorname{Diff}(M)$ consists of all vector fields $X$ on $M$ such that $\left.X\right|_{\partial^{q} M}$ is tangent to $\partial^{q} M$. We shall denote this Lie algebra by $\mathfrak{X}(M, \partial M)$.

3.2. Lemma. Any manifold with corners $M$ is a submanifold with corners of an open manifold $\tilde{M}$ of the same dimension, and each smooth function on $M$ extends to a smooth function on $\tilde{M}$. Each smooth vector bundle over $M$ extends to a smooth vector bundle over $\tilde{M}$. Each immersion (embedding) of $M$ into a smooth manifold $N$ without boundary is the restriction of an immersion (embedding) of a (possibly smaller) $\tilde{M} \supset M$ into $N$.

Proof. Choose a vector field $X$ on $M$ which is complete, and along $\partial M$ is nowhere 0 and pointing into the interior. Then for $\varepsilon>0$ we can replace $M$ by the flow image $\mathrm{Fl}_{\varepsilon}^{X}(M)$ which is contained in the interior $\tilde{M}=M \backslash \partial M$. The extension properties follow from the Whitney extension theorem. An immersion extends, since its rank cannot fall locally. An embedding $f$ extends since $\left\{(f(x), f(y)):(x, y) \in M \times M \backslash \operatorname{Diag}_{M}\right\}$ has positive distance to the closed $\operatorname{Diag}_{N}$ in $N \times N$, locally in $M$, and we can keep it that way; see 69. $5.3]$ for too many details.

3.3. Differential forms on manifolds with corners. There are several differential complexes on a manifold with corners. If $M$ is not compact there are also the versions with compact support.

- Differential forms that vanish near $\partial M$. If $M$ is compact, this is the same as the differential complex $\Omega_{c}(M \backslash \partial M)$ of differential forms with compact support in the open interior $M \backslash \partial M$.

- $\Omega(M, \partial M)=\left\{\alpha \in \Omega(M): j_{\partial^{q} M}^{*} \alpha=0\right.$ for all $\left.q \geq 1\right\}$, the complex of differential forms that pull back to 0 on each boundary stratum.

- $\Omega(M)$, the complex of all differential forms. Its cohomology equals singular cohomology with real coefficients of $M$, since $\mathbb{R} \rightarrow \Omega^{0} \rightarrow$ $\Omega^{1} \rightarrow \ldots$ is a fine resolution of the constant sheaf on $M$; for that one needs existence of smooth partitions of unity and the Poincaré lemma which hold on manifolds with corners. The Poincaré lemma can be proved as in [73, 9.10] in each quadrant. 
If $M$ is an oriented manifold with corners of dimension $m$ and if $\mu \in \Omega^{m}(M)$ is a nowhere vanishing form of top degree, then $\mathfrak{X}(M) \ni X \mapsto i_{X} \mu \in \Omega^{m-1}(M)$ is a linear isomorphism. Moreover, $X \in \mathfrak{X}(M, \partial M)$ (tangent to the boundary) if and only if $i_{X} \mu \in \Omega^{m-1}(M, \partial M)$.

3.4. Towards the long exact sequence of the pair $(M, \partial M)$. Let us consider the short exact sequence of differential graded algebras

$$
0 \rightarrow \Omega(M, \partial M) \rightarrow \Omega(M) \rightarrow \Omega(M) / \Omega(M, \partial M) \rightarrow 0
$$

The complex $\Omega(M) / \Omega(M, \partial M)$ is a subcomplex of the product of $\Omega(N)$ for all connected components $N$ of all $\partial^{q} M$. The quotient consists of forms which extend continuously over boundaries to $\partial M$ with its induced topology in such a way that one can extend them to smooth forms on $M$; this is contained in the space of 'stratified forms' as used in [104. There Stokes' formula is proved for stratified forms.

3.5. Proposition (Stokes' theorem). For a connected oriented manifold $M$ with corners of dimension $\operatorname{dim}(M)=m$ and for any $\omega \in \Omega_{c}^{m-1}(M)$ we have

$$
\int_{M} d \omega=\int_{\partial^{1} M} j_{\partial^{1} M}^{*} \omega
$$

Note that $\partial^{1} M$ may have several components. Some of these might be non-compact.

We shall deduce this result from Stokes' formula for a manifold with boundary by making precise the fact that $\partial^{\geq 2} M$ has codimension 2 in $M$ and has codimension 1 with respect to $\partial^{1} M$. The proof also works for manifolds with more general boundary strata, like manifolds with cone-like singularities. A lengthy full proof can be found in [24].

Proof. We first choose a smooth decreasing function $f$ on $\mathbb{R}_{\geq 0}$ such that $f=1$ near 0 and $f(r)=0$ for $r \geq \varepsilon$. Then $\int_{0}^{\infty} f(r) d r<\varepsilon$ and for $Q^{m}=\mathbb{R}_{\geq 0}^{m}$ with $m \geq 2$,

$$
\begin{aligned}
\left|\int_{Q^{m}} f^{\prime}(|x|) d x\right| & =C_{m}\left|\int_{0}^{\infty} f^{\prime}(r) r^{m-1} d r\right|=C_{m}\left|\int_{0}^{\infty} f(r)\left(r^{m-1}\right)^{\prime} d r\right| \\
& =C_{m} \int_{0}^{\varepsilon} f(r)\left(r^{m-1}\right)^{\prime} d r \leq C_{m} \varepsilon^{m-1}
\end{aligned}
$$

where $C_{m}$ denotes the surface area of $S^{m-1} \cap Q^{m}$. Given $\omega \in \Omega_{c}^{m-1}(M)$ we use the function $f$ on quadrant charts on $M$ to construct a function $g$ on $M$ that is 1 near $\partial^{\geq 2} M=\bigcup_{q \geq 2} \partial^{q} M$, has support close to $\partial^{\geq 2} M$ and satisfies $\left|\int_{M} d g \wedge \omega\right|<\varepsilon$. Then $(1-g) \omega$ is an $(m-1)$-form with compact support in the manifold with boundary $M \backslash \partial^{\geq 2} M$, and Stokes' formula (cf. [73, 10.11]) now says

$$
\int_{M \backslash \partial \geq^{2} M} d((1-g) \omega)=\int_{\partial^{1} M} j_{\partial^{1} M}^{*}((1-g) \omega) .
$$


But $\partial^{\geq 2} M$ is a null set in $M$ and the quantities

$$
\left|\int_{M} d((1-g) \omega)-\int_{M} d \omega\right| \text { and }\left|\int_{\partial^{1} M} j_{\partial^{1} M}^{*}((1-g) \omega)-\int_{\partial^{1} M} j_{\partial^{1} M}^{*} \omega\right|
$$

are small if $\varepsilon$ is small enough.

3.6. Riemannian manifolds with bounded geometry. If $M$ is not necessarily compact without boundary we equip $M$ with a Riemannian metric $g$ of bounded geometry which exists by [47, Theorem 2']. This means that

$(I) \quad$ The injectivity radius of $(M, g)$ is positive.

$\left(B_{\infty}\right) \quad$ Each iterated covariant derivative of the curvature is uniformly $g$-bounded: $\left\|\nabla^{i} R\right\|_{g}<C_{i}$ for $i=0,1,2, \ldots$

The following is a compilation of special cases of results collected in 30 , chapter 1].

Proposition ([53, [29]). If $(M, g)$ satisfies $(I)$ and $\left(B_{\infty}\right)$ then the following holds

(1) $(M, g)$ is complete.

(2) There exists $\varepsilon_{0}>0$ such that for each $\varepsilon \in\left(0, \varepsilon_{0}\right)$ there is a countable cover of $M$ by geodesic balls $B_{\varepsilon}\left(x_{\alpha}\right)$ such that the cover of $M$ by the balls $B_{2 \varepsilon}\left(x_{\alpha}\right)$ is still uniformly locally finite.

(3) Moreover there exists a partition of unity $1=\sum_{\alpha} \rho_{\alpha}$ on $M$ such that $\rho_{\alpha} \geq 0, \rho_{\alpha} \in C_{c}^{\infty}(M), \operatorname{supp}\left(\rho_{\alpha}\right) \subset B_{2 \varepsilon}\left(x_{\alpha}\right)$, and $\left|D_{u}^{\beta} \rho_{\alpha}\right|<C_{\beta}$ where $u$ are normal (Riemannian exponential) coordinates in $B_{2 \varepsilon}\left(x_{\alpha}\right)$.

(4) In each $B_{2 \varepsilon}\left(x_{\alpha}\right)$, in normal coordinates, we have $\left|D_{u}^{\beta} g_{i j}\right|<C_{\beta}^{\prime}$, $\left|D_{u}^{\beta} g^{i j}\right|<C_{\beta}^{\prime \prime}$, and $\left|D_{u}^{\beta} \Gamma_{i j}^{m}\right|<C_{\beta}^{\prime \prime \prime}$, where all constants are independent of $\alpha$.

3.7. Riemannian manifolds with bounded geometry allowing corners. If $M$ has corners, we choose an open manifold $\tilde{M}$ of the same dimension which contains $M$ as a submanifold with corners; see 3.1. It is very desirable to prove that then there exists a Riemannian metric $\tilde{g}$ on $\tilde{M}$ with bounded geometry such that each boundary component of each $\partial^{q} M$ is totally geodesic.

For a compact manifold with boundary (no corners of codimension $\geq 2$ ), existence of such a Riemannian metric was proven in [45, 2.2.3] in a more complicated context. A simple proof goes as follows: Choose a tubular neighborhood $U$ of $\partial M$ in $\tilde{M}$ and use the symmetry $\varphi(u)=-u$ in the vector bundle structure on $U$. Given a metric $\tilde{g}$ on $\tilde{M}$, then $\partial M$ is totally geodesic for the metric $\frac{1}{2}\left(\tilde{g}+\varphi^{*} \tilde{g}\right)$ on $U$, since $\partial M$ (the zero section) is the fixed point set of the isometry $\varphi$. Now glue this metric to the $\tilde{g}$ using a partition of unity for the cover $U$ and $\tilde{M} \backslash V$ for a closed neighborhood $V$ of $\partial M$ in $U$.

Existence of a geodesic spray on a manifold with corners which is tangential to each boundary component $\partial^{q} M$ was proved in [69, 2.8, see also 10.3]. A direct proof of this fact can be distilled from the proof of lemma 5.9 below. This is sufficient for constructing charts on the diffeomorphism group Diff( $(M)$ in 6.1 below. 


\section{WHITNEY MANIFOLD GERMS}

More general than manifolds with corners, Whitney manifold germs allow for quite singular boundaries but still controlled enough so that a continuous Whitney extension operator to an open neighborhood manifold exists.

4.1. Compact Whitney subsets. Let $\tilde{M}$ be an open smooth connected $m$ dimensional manifold. A closed connected subset $M \subset \tilde{M}$ is called a Whitney subset, or $\tilde{M} \supset M$ is called a Whitney pair, if

(1) $M$ is the closure of its open interior in $\tilde{M}$, and:

(2) There exists a continuous linear extension operator

$$
\mathcal{E}: \mathcal{W}(M) \rightarrow C^{\infty}(\tilde{M}, \mathbb{R})
$$

from the linear space $\mathcal{W}(M)$ of all Whitney jets of infinite order with its natural Fréchet topology (see below) into the space $C^{\infty}(\tilde{M}, \mathbb{R})$ of smooth functions on $\tilde{M}$ with the Fréchet topology of uniform convergence on compact subsets in all derivatives separately.

We speak of a compact Whitney subset or compact Whitney pair if $M$ is compact. In this case, in (2), we may equivalently require that $\mathcal{E}$ is linear continuous into the Fréchet space $C_{L}^{\infty}(\tilde{M}, \mathbb{R}) \subset C_{c}^{\infty}(\tilde{M}, \mathbb{R})$ of smooth functions with support in a compact subset $L$ which contains $M$ in its interior, by using a suitable bump function.

The property of being a Whitney pair is obviously invariant under diffeomorphisms (of $\tilde{M}$ ) which act linearly and continuously both on $\mathcal{W}(M)$ and $C^{\infty}(\tilde{M}, \mathbb{R})$ in a natural way.

This property of being a Whiney pair is local in the following sense: If $\tilde{M}_{i} \supset M_{i}$ covers $\tilde{M} \supset M$, then $\tilde{M} \supset M$ is a Whitney pair if and only if each $\tilde{M}_{i} \supset M_{i}$ is a Whitney pair, see Theorem 4.4 below.

More details: For $\mathbb{R}^{m} \supset M$, by an extension operator $\mathcal{E}: \mathcal{W}(M) \rightarrow$ $C^{\infty}(\tilde{M}, \mathbb{R})$ we mean that $\left.\partial_{\alpha} \mathcal{E}(F)\right|_{M}=F^{(\alpha)}$ for each multi-index $\alpha \in \mathbb{N}_{\geq 0}^{m}$ and each Whitney jet $F \in \mathcal{W}(M)$. We recall the definition of a Whitney jet $F$. If $M \subset \mathbb{R}^{m}$ is compact, then

$$
\begin{aligned}
& F=\left(F^{(\alpha)}\right)_{\alpha \in \mathbb{N}_{\geq 0}^{m}} \in \prod_{\alpha} C^{0}(M) \quad \text { such that for } \\
& T_{y}^{n}(F)(x)=\sum_{|\alpha| \leq n} \frac{F^{(\alpha)}(y)}{\alpha !}(x-y)^{\alpha} \quad \text { the remainder seminorm }
\end{aligned}
$$

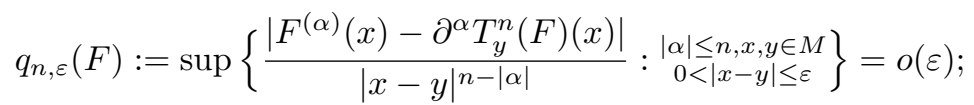

so $q_{n, \varepsilon}(F)$ goes to 0 for $\varepsilon \rightarrow 0$, for each $n$ separately. The $n$-th Whitney seminorm is then

$$
\|F\|_{n}=\sup \left\{\left|F^{(\alpha)}(x)\right|: x \in M,|\alpha| \leq n\right\}+\sup \left\{q_{n, \varepsilon}(F): \varepsilon>0\right\} .
$$

For closed but non-compact $M$ one uses the projective limit over a countable compact exhaustion of $M$. This describes the natural Fréchet topology on the 
space of Whitney jets for closed subsets of $\mathbb{R}^{m}$. The extension to manifolds is obvious.

Whitney proved in 107 that a linear extension operator always exists for a closed subset $M \subset \mathbb{R}^{m}$, but not always a continuous one, for example for $M$ a point. For a finite differentiability class $C^{n}$ there exists always a continuous extension operator.

4.2. Proposition. For a Whitney pair $\tilde{M} \supset M$, the space of $\mathcal{W}(M)$ of Whitney jets on $M$ is linearly isomorphic to the space

$$
C^{\infty}(M, \mathbb{R}):=\left\{\left.f\right|_{M}: f \in C^{\infty}(\tilde{M}, \mathbb{R})\right\} .
$$

Proof. This follows from [40, 3.11], where the following is proved: If $f \in$ $C^{\infty}\left(\mathbb{R}^{m}, \mathbb{R}\right)$ vanishes on a Whitney subset $M \subset \mathbb{R}^{m}$, then $\left.\partial^{\alpha} f\right|_{M}=0$ for each multi-index $\alpha$. Thus any continuous extension operator is injective.

4.3. Examples and counterexamples of Whitney pairs. We collect here results about closed subsets of $\mathbb{R}^{m}$ which are or are not Whitney subsets.

(a) If $M$ is a manifold with corners, then $\tilde{M} \supset M$ is a Whitney pair. This follows from Mityagin [79] or Seeley 97.

(b) If $M$ is closed in $\mathbb{R}^{m}$ with dense interior and with Lipschitz boundary, then $\mathbb{R}^{m} \supset M$ is a Whitney pair; by [99, p 181]. In [15, Theorem I] Bierstone proved that a closed subset $M \subset \mathbb{R}^{n}$ with dense interior is a Whitney pair, if it has Hölder $C^{0, \alpha}$-boundary for $0<\alpha \leq 1$ which may be chosen on each $M \cap\{x: N \leq|x| \leq N+2\}$ separately. A fortiori, each subanalytic subset in $\mathbb{R}^{n}$ gives a Whitney pair, [15, Theorem II].

(c) If $f \in C^{\infty}\left(\mathbb{R}_{>0}, \mathbb{R}\right.$ ) which is flat at 0 (all derivatives vanish at 0 ), and if $M$ is a closed subset containing 0 of $\{(x, y): x \geq 0,|y| \leq|f(x)|\} \subset \mathbb{R}^{2}$, then $\mathbb{R}^{2} \supset M$ is not a Whitney pair; see [101, Beispiel 2].

(d) For $r \geq 1$, the set $\left\{x \in \mathbb{R}^{m}: 0 \leq x_{1} \leq 1, x_{2}^{2}+\cdots+x_{m}^{2} \leq x_{1}^{2 r}\right\}$ is called the parabolic cone of order $r$. Then the following result [101, Satz 4.6] holds: $A$ closed subset $M \subset \mathbb{R}^{m}$ is a Whitney subset, if the following condition holds: For each compact $K \subset \mathbb{R}^{m}$ there exists a parabolic cone $S$ and a family $\varphi_{i}: S \rightarrow \phi_{i}(S) \subset M \subset \mathbb{R}^{m}$ of diffeomorphisms such that $K \cap M \subseteq \bigcup_{i} \overline{\varphi_{i}(S)}$ and $\sup _{i}\left|\varphi_{i}\right|_{k}<\infty, \sup _{i}\left|\varphi_{i}^{-1}\right|_{k}<\infty$ for each $k$ separately.

(e) A characterization of closed subsets admitting continuous Whitney extension operators has been found by Frerick [40, 4.11] in terms of local Markov inequalities, which however, is very difficult to check directly.

Let $M \subset \mathbb{R}^{m}$ be closed. Then the following are equivalent:

(e1) $M$ admits a continuous linear Whitney extension operator

$$
\mathcal{E}: \mathcal{W}(M) \rightarrow C^{\infty}\left(\mathbb{R}^{m}, \mathbb{R}\right) .
$$

(e2) For each compact $K \subset M$ and $\theta \in(0,1)$ there is $r \geq 0$ and $\varepsilon_{0}>0$ such that for all $k \in \mathbb{N}_{\geq 1}$ there is $C \geq 1$ such that

$$
\left|d p\left(x_{0}\right)\right| \leq \frac{C}{\varepsilon^{r}} \sup _{\substack{\left|y-x_{0}\right| \leq \varepsilon \\ y \in \mathbb{R}^{m}}}|p(y)|^{\theta} \sup _{\substack{\left|x-x_{0}\right| \leq \varepsilon \\ x \in M}}|p(x)|^{1-\theta}
$$


for all $p \in \mathbb{C}\left[x_{1}, \ldots, x_{m}\right]$ of degree $\leq k$, for all $x_{0} \in K$, and for all $\varepsilon_{0}>\varepsilon>0$.

(e3) For each compact $K \subset M$ there exists a compact $L$ in $\mathbb{R}^{m}$ containing $K$ in its interior, such that for all $\theta \in(0,1)$ there is $r \geq 1$ and $C \geq 1$ such that

$$
\sup _{x \in K}|d p(x)| \leq C \operatorname{deg}(p)^{r} \sup _{y \in L}|p(y)|^{\theta} \sup _{z \in L \cap M}|p(z)|^{1-\theta}
$$

for all $p \in \mathbb{C}\left[x_{1}, \ldots, x_{m}\right]$.

(f) Characterization (e) has been generalized to a characterization of compact subsets of $\mathbb{R}^{m}$ which admit a continuous Whitney extension operator with linear (or even affine) loss of derivatives, in [41]. In the paper [42] a similar characterization is given for an extension operator without loss of derivative, and a sufficient geometric condition is formulated [42, Corollary 2] which even implies that there are closed sets with empty interior admitting continuous Whitney extension operators, like the Sierpiński triangle or Cantor subsets. Thus we cannot omit assumption 4.1.1) that $M$ is the closure of its open interior in $\tilde{M}$ in our definition of Whitney pairs.

(g) The following result by Frerick [40, Theorem 3.15] gives an easily verifiable sufficient condition:

Let $K \subset \mathbb{R}^{m}$ be compact and assume that there exist $\varepsilon_{0}>0, \rho>0, r \geq 1$ such that for all $z \in \partial K$ and $0<\varepsilon<\varepsilon_{0}$ there is $x \in K$ with $B_{\rho \varepsilon^{r}}(x) \subset$ $K \cap B_{\varepsilon}(z)$. Then $K$ admits a continuous linear Whitney extension operator $\mathcal{W}(F) \rightarrow C^{\infty}\left(\mathbb{R}^{m}, \mathbb{R}\right)$.

This implies (a), (b), and (d).

4.4. Theorem. Let $\tilde{M}$ be an open manifold and let $M \subset \tilde{M}$ be a compact subset that is the closure of its open interior. $M \subset \tilde{M}$ is a Whitney pair if and only if for every smooth atlas $\left(\tilde{M} \supset U_{\alpha}, u_{\alpha}: U_{\alpha} \rightarrow u_{\alpha}\left(U_{\alpha}\right) \subset \mathbb{R}^{m}\right)_{\alpha \in A}$ of the open manifold $\tilde{M}$, each $u_{\alpha}\left(M \cap U_{\alpha}\right) \subset u_{\alpha}\left(U_{\alpha}\right)$ is a Whitney pair.

Consequently, for a Whitney pair $M \subset \tilde{M}$ and $U \subset \tilde{M}$ open, $M \cap U \subset$ $\tilde{M} \cap U$ is also a Whitney pair.

Proof. (1) We consider a locally finite countable smooth atlas $\left(\tilde{M} \supset U_{\alpha}, u_{\alpha}\right.$ : $\left.U_{\alpha} \rightarrow u_{\alpha}\left(U_{\alpha}\right) \subset \mathbb{R}^{m}\right)_{\alpha \in \mathbb{N}}$ of $\tilde{M}$ such that each $u_{\alpha}\left(U_{\alpha}\right) \supset u_{\alpha}\left(M \cap U_{\alpha}\right)$ is a Whitney pair.

We use a smooth 'partition of unity' $f_{\alpha} \in C_{c}^{\infty}\left(U_{\alpha}, \mathbb{R}_{\geq 0}\right)$ on $\tilde{M}$ with $\sum_{\alpha} f_{\alpha}^{2}=1$. The following mappings induce linear embeddings onto closed direct summands of the Fréchet spaces:

$$
\begin{gathered}
C^{\infty}(\tilde{M}, \mathbb{R}) \underset{\sum_{\alpha} f_{\alpha} \cdot g_{\alpha} \leftarrow\left(g_{\alpha}\right)_{\alpha}}{\rightleftarrows} \prod_{\alpha} C^{\infty}\left(U_{\alpha}, \mathbb{R}\right) \\
\mathcal{W}(M) \stackrel{\longrightarrow}{\longleftarrow} \prod_{\alpha} \mathcal{W}\left(U_{\alpha} \cap M\right)
\end{gathered}
$$


If each $u_{\alpha}\left(U_{\alpha}\right) \supset u_{\alpha}\left(U_{\alpha} \cap M\right)$ is a Whitney pair, then so is $U_{\alpha} \supset U_{\alpha} \cap M$, via the isomorphisms induced by $u_{\alpha}$, and

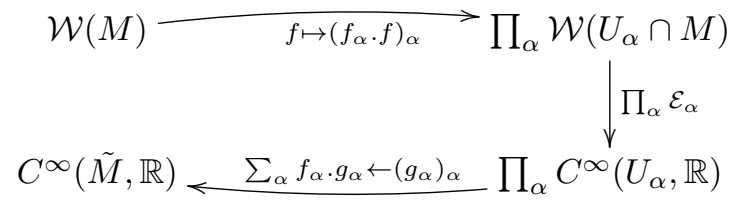

is a continuous Whitney extension operator, so that $\tilde{M} \supset M$ is a Whitney pair. This proves the easy direction of the theorem.

The following argument for the converse direction is inspired by [43]. (2) (See [40, Def. 3.1], 65, Section 29-31]) A Fréchet space $E$ is said to have property $(\mathrm{DN})$ if for one (equivalently, any) increasing system $\left(\|\cdot\|_{n}\right)_{n \in \mathbb{N}}$ of seminorms generating the topology the following holds:

- There exists a continuous seminorm \| $\|$ on $E$ (called a dominating norm, hence the name (DN)) such that for all (equivalently, one) $0<\theta<1$ and all $m \in \mathbb{N}$ there exist $k \in \mathbb{N}$ and $C>0$ with

$$
\|\|_{m} \leq C\|\|_{k}^{\theta} \cdot\|\|^{1-\theta} \text {. }
$$

The property $(\mathrm{DN})$ is an isomorphism invariant and is inherited by closed linear subspaces. The Fréchet space $\mathfrak{s}$ of rapidly decreasing sequences has property $(\mathrm{DN})$.

(3) ([101, Satz 2.6], see also [40, Theorem 3.3]) A closed subset $M$ in $\mathbb{R}^{m}$ admits a continuous linear extension operator $\mathcal{W}(M) \rightarrow C^{\infty}\left(\mathbb{R}^{m}, \mathbb{R}\right)$ if and only if for each $x \in \partial M$ there exists a compact neighborhood $K$ of $x$ in $\mathbb{R}^{m}$ such that

$$
\mathcal{W}_{K}(M):=\left\{f \in \mathcal{W}(M): \operatorname{supp}\left(f^{(\alpha)}\right) \subset K \text { for all } \alpha \in \mathbb{N}_{\geq 0}^{m}\right\} .
$$

has property (DN).

We assume from now on that $\tilde{M} \supset M$ is a Whitney pair.

(4) Given a compact set $K \subset \tilde{M}$, let $L \subset \tilde{M}$ be a compact smooth manifold with smooth boundary which contains $K$ in its interior. Let $\tilde{L}$ be the double of $L$, i.e., $L$ smoothly glued to another copy of $L$ along the boundary; $\tilde{L}$ is a compact smooth manifold containing $L$ as a submanifold with boundary.

Then $C^{\infty}(\tilde{L}, \mathbb{R})$ is isomorphic to the space $\mathfrak{s}$ of rapidly decreasing sequences: This is due to [105]. In fact, using a Riemannian metric $g$ on $\tilde{L}$, the expansion in an orthonormal basis of eigenvectors of $1+\Delta^{g}$ of a function $h \in L^{2}$ has coefficients in $\mathfrak{s}$ if and only if $h \in C^{\infty}(\tilde{L}, \mathbb{R})$, because $1+\Delta^{g}: H^{k+2}(\tilde{L}) \rightarrow H^{k}(\tilde{L})$ is an isomorphism for Sobolev spaces $H^{k}$ with $k \geq 0$, and since the eigenvalues $\mu_{n}$ of $\Delta^{g}$ satisfy $\mu_{n} \sim C_{\tilde{L}} \cdot n^{2 / \operatorname{dim}(\tilde{L})}$ for $n \rightarrow \infty$, by Weyl's asymptotic formula. Thus $C^{\infty}(\tilde{L}, \mathbb{R})$ has property (DN).

Moreover, $C_{L}^{\infty}(\tilde{M}, \mathbb{R})=\left\{f \in C^{\infty}(\tilde{M}, \mathbb{R}): \operatorname{supp}(f) \subset L\right\}$ is a closed linear subspace of $C^{\infty}(\tilde{L}, \mathbb{R})$, by extending each function by 0 . Thus also $C_{L}^{\infty}(\tilde{M}, \mathbb{R})$ has property (DN). 
We choose now a function $g \in C_{L}^{\infty}\left(\tilde{M}, \mathbb{R}_{\geq 0}\right)$ with $\left.g\right|_{K}=1$ and consider:

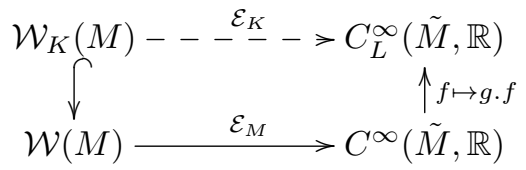

The resulting composition $\mathcal{E}_{K}$ is a continuous linear embedding onto a closed linear subspace of the space $C_{L}^{\infty}(\tilde{M}, \mathbb{R})$ which has $(\mathrm{DN})$. Thus we proved:

(5) Claim. If $\tilde{M} \supset M$ is a Whitney pair and $K$ is compact in $\tilde{M}$, the Fréchet space $\mathcal{W}_{K}(M)$ has property $(\mathrm{DN})$.

(6) We consider now a smooth chart $\tilde{M} \supset U \stackrel{u}{\rightarrow} u(U)=\mathbb{R}^{m}$. For $x \in \partial u(M)$ let $K$ be a compact neighborhood of $x$ in $\mathbb{R}^{m}$. The chart $u$ induces a linear isomorphism

$$
\mathcal{W}_{K}(u(M \cap U)) \stackrel{u^{*}}{\longrightarrow} \mathcal{W}_{u^{-1}(K)}(U \cap M) \cong \mathcal{W}_{u^{-1}(K)}(M),
$$

where the right-hand side mapping is given by extending each $f^{(\alpha)}$ by 0 . By claim (5) the Fréchet space $W_{u^{-1}(K)}(M)$ has property (DN); consequently also the isomorphic space $\mathcal{W}_{K}(u(M \cap U))$ has property (DN). By (3) we conclude that $\mathbb{R}^{m}=u(U) \supset u(M \cap U)$ is a Whitney pair.

(7) If we are given a general chart $\tilde{M} \supset U \stackrel{u}{\rightarrow} u(U) \subset \mathbb{R}^{m}$, we cover $U$ by a locally finite atlas $\left(U \supset U_{\alpha}, u_{\alpha}: U_{\alpha} \rightarrow u_{\alpha}\left(U_{\alpha}\right)=\mathbb{R}^{m}\right)_{\alpha \in \mathbb{N}}$. By (6) each $\mathbb{R}^{m}=u_{\alpha}\left(U_{\alpha}\right) \supset u_{\alpha}\left(M \cap U_{\alpha}\right)$ is a Whitney pair, and by the argument in (1) the pair $U \supset M \cap U$ is a Whitney pair, and thus the diffeomorphic $u(U) \supset u(U \cap M)$ is also a Whitney pair.

4.5. Our use of Whitney pairs. We consider an equivalence class of Whitney pairs $\tilde{M}_{i} \supset M_{i}$ for $i=0,1$ where $\tilde{M}_{0} \supset M_{0}$ is equivalent to $\tilde{M}_{1} \supset M_{1}$ if there exist an open submanifolds $\tilde{M}_{i} \supset \hat{M}_{i} \supset M_{i}$ and a diffeomorphism $\varphi: \hat{M}_{0} \rightarrow \hat{M}_{1}$ with $\varphi\left(M_{0}\right)=M_{1}$. By a germ of a Whitney manifold we mean an equivalence class of Whitney pairs as above. Given a Whitney pair $\tilde{M} \supset M$ and its corresponding germ, we may keep $M$ fixed and equip it with all open connected neighborhoods of $M$ in $\tilde{M}$; each neighborhood is then a representative of this germ; called an open neighborhood manifold of $M$. In the following we shall speak of a Whitney manifold germ $M$ and understand that it comes with open manifold neighborhoods $\tilde{M}$. If we want to stress a particular neighborhood we will write $\tilde{M} \supset M$.

The boundary $\partial M$ of a Whitney manifold germ is the topological boundary of $M$ in $\tilde{M}$. It can be a quite general set as seen from the examples 4.3 and the discussion in 4.9. But infinitely flat cusps cannot appear.

4.6. Other approaches. We remark that there are other settings, like the concept of a manifold with rough boundary; see 95. and literature cited there. The main idea there is to start with closed subsets $C \subset \mathbb{R}^{m}$ with dense interior, to use the space of functions which are $C^{n}$ in the interior of $C$ such that all partial derivatives extend continuously to $C$. Then one looks for 
sufficient conditions (in particular for $n=\infty$ ) on $C$ such that there exists a continuous Whitney extension operator on the space of these functions, and builds manifolds from that. The condition in [95] are in the spirit of [4.3.d). By extending these functions and restricting their jets to $C$ we see that the manifolds with rough boundary are Whitney manifold germs.

Another possibility is to consider closed subsets $C \subset \mathbb{R}^{m}$ with dense interior such there exists a continuous linear extension operator on the space $C^{\infty}(C)=\left\{\left.f\right|_{C}: f \in C^{\infty}\left(\mathbb{R}^{m}\right)\right\}$ with the quotient locally convex topology. These are exactly the Whitney manifold pairs $\mathbb{R}^{m} \supset M$, by 4.2 . In this setting, for $C^{n}$ with $n<\infty$ there exist continuous extension operators $C_{b}^{n}(C) \rightarrow C_{b}^{n}\left(\mathbb{R}^{m}\right)$ (where the subscript $b$ means bounded for all derivatives separately) for arbitrary subsets $C \subset \mathbb{R}^{m}$; see 39 .

We believe that our use of Whitney manifold germs is quite general, simple, and avoids many technicalities. But it is aimed at the case $C^{\infty}$; for $C^{k}$ or $W^{k, p}$ other approaches, like the one in [95], might be more appropriate.

4.7. Tangent vectors and vector fields on Whitney manifold germs. In line with the more general convention for vector bundles in 4.8 below, we define the tangent bundle $T M$ of a Whitney manifold germ $M$ as the restriction $T M=\left.T \tilde{M}\right|_{M}$. For $x \in \partial M$, a tangent vector $X_{x} \in T_{x} M$ is said to be interior pointing if there exist a curve $c:[0,1) \rightarrow M$ which is smooth into $\tilde{M}$ with $c^{\prime}(0)=X_{x}$. And $X_{x} \in T_{x} M$ is called tangent to the boundary if there exists a curve $c:(-1,1) \rightarrow \partial M$ which is smooth into $\tilde{M}$ with $c^{\prime}(0)=X_{x}$. The space of vector fields on $M$ is given as

$$
\mathfrak{X}(M)=\left\{\left.X\right|_{M}: X \in \mathfrak{X}(\tilde{M})\right\} .
$$

Using a continuous linear extension operator, $\mathfrak{X}(M)$ is isomorphic to a locally convex direct summand in $\mathfrak{X}(\tilde{M})$. If $M$ is a compact Whitney manifold germ, $\mathfrak{X}(M)$ is a direct summand even in $\mathfrak{X}_{L}(\tilde{M})=\{X \in \mathfrak{X}(\tilde{M}): \operatorname{supp}(X) \subseteq L\}$ where $L \subset \tilde{M}$ is a compact set containing $M$ in its interior. We define the space of vector fields on $M$ which are tangent to the boundary as

$$
\begin{aligned}
& \mathfrak{X}_{\partial}(M)=\left\{\left.X\right|_{M}: X \in \mathfrak{X}(\tilde{M}), x \in \partial M \Longrightarrow \mathrm{Fl}_{t}^{X}(x) \in \partial M\right. \\
& \left.\qquad \text { for all } t \text { for which } \mathrm{Fl}_{t}^{X}(x) \text { exists in } \tilde{M}\right\},
\end{aligned}
$$

where $\mathrm{Fl}_{t}^{X}$ denotes the flow mapping of the vector field $X$ up to time $t$ which is locally defined on $\tilde{M}$. Obviously, for $X \in \mathfrak{X}_{\partial}(M)$ and $x \in \partial M$ the tangent vector $X(x)$ is tangent to the boundary in the sense defined above. I have no proof that the converse is true:

Question. Suppose that $X \in \mathfrak{X}(\tilde{M})$ has the property that for each $x \in \partial M$ the tangent vector $X(x)$ is tangent to the boundary. Is it true that then $\left.X\right|_{M} \in \mathfrak{X}_{\partial}(M)$ ?

A related question for which I have no answer is:

Question. For each $x \in \partial M$ and tangent vector $X_{x} \in T_{x} M$ which is tangent to the boundary, is there a smooth vector field $X \in \mathfrak{X}_{c, \partial}(M)$ with $X(x)=X_{x}$ ? 
Lemma. For a Whitney manifold germ $M$, the space $\mathfrak{X}_{\partial}(M)$ of vector field tangent to the boundary is a closed linear sub Lie algebra of $\mathfrak{X}(M)$. The space $\mathfrak{X}_{c, \partial}(M)$ of vector fields with compact support tangent to the boundary is a closed linear sub Lie algebra of $\mathfrak{X}_{c}(M)$.

Proof. By definition, for $X \in \mathfrak{X}(\tilde{M})$ the restriction $\left.X\right|_{M}$ is in $\mathfrak{X}_{\partial}(M)$ if and only if $x \in \partial M$ implies that $\mathrm{Fl}_{t}^{X}(x) \in \partial M$ for all $t$ for which $\mathrm{Fl}_{t}^{X}(x)$ exists in $\tilde{M}$. These conditions describe a set of continuous equations, since $(X, t, x) \mapsto \mathrm{Fl}_{t}^{X}(x)$ is smooth; see the proof of 6.1 for a simple argument. Thus $X \in \mathfrak{X}(\tilde{M})$ is closed.

The formulas (see, e.g., [81, p 56,58])

$$
\begin{gathered}
\lim _{n \rightarrow \infty}\left(F l_{t / n}^{X} \circ \mathrm{Fl}_{t / n}^{Y}\right)^{n}(x)=\mathrm{Fl}_{t}^{X+Y}(x) \\
\lim _{n \rightarrow \infty}\left(\mathrm{Fl}_{-(t / n)^{1 / 2}}^{Y} \circ \mathrm{Fl}_{-(t / n)^{1 / 2}}^{X} \circ \mathrm{Fl}_{(t / n)^{1 / 2}}^{Y} \circ \mathrm{Fl}_{(t / n)^{1 / 2}}^{X}\right)^{n}(x)=\mathrm{Fl}_{t}^{[X, Y]}(x)
\end{gathered}
$$

shows that $\mathfrak{X}_{\partial}(M)$ is a Lie subalgebra.

The smooth partial stratifications of the boundary of a Whitney manifold germ. Given a Whitney manifold germ $\tilde{M} \supset M$ of dimension $m$, for each $x \in \partial M$ we denote by $\mathcal{L}^{\infty}(x)$ the family consisting of each maximal connected open smooth submanifold $L$ of $\tilde{M}$ which contains $x$ and is contained in $\partial M$. Note that for $L \in \mathcal{L}^{\infty}(x)$ and $y \in L$ we have $L \in \mathcal{L}^{\infty}(y)$. $\left\{T_{x} L: L \in \mathcal{L}^{\infty}(x)\right\}$ is a set of linear subspaces of $T_{x} \tilde{M}$. The collective of these for all $x \in \partial M$ is something like a 'field of quivers of vector spaces' over $\partial M$. It might be the key to eventually construct charts for the regular Frölicher Lie group Diff $(M)$ treated in 6.3 below, and for constructing charts for the Frölicher space $\operatorname{Emb}(M, N)$ in 7.2 below.

4.8. Mappings, bundles, and sections. Let $M$ be Whitney manifold germ and let $N$ be a manifold without boundary. By a smooth mapping $f: M \rightarrow N$ we mean $f=\left.\tilde{f}\right|_{M}$ for a smooth mapping $\tilde{f}: \tilde{M} \rightarrow N$ for an open manifold neighborhood $\tilde{M} \supset M$. Whitney jet on $M$ naively make sense only if they take values in a vector space or, more generally, in a vector bundle. One could develop the notion of Whitney jets of infinite order with values in a manifold as sections of $J^{\infty}(M, N) \rightarrow M$ with Whitney conditions of each order. We do not know whether this has been written down formally. But we can circumvent this easily by considering a closed embedding $i: N \rightarrow \mathbb{R}^{p}$ and a tubular neighborhood $p: U \rightarrow i(N)$; i.e., $U$ is an open neighborhood and is (diffeomorphic to) the total space of a smooth vector bundle which projection $p$.

Then we can consider a Whitney jet on $M$ with values in $\mathbb{R}^{p}$ (in other words, a $p$-tuple of Whitney jets) such that the 0 order part lies in $i(N)$. Using a continuous Whitney extension operator, we can extend the Whitney jet to a smooth mapping $\tilde{f}: \tilde{M} \rightarrow \mathbb{R}^{p}$. Then consider the open set $\tilde{f}^{-1}(U) \subset \tilde{M}$ instead of $\tilde{M}$, and replace $\tilde{f}$ by $p \circ \tilde{f}$. So we just extended the given Whitney 
jet to a smooth mapping $\tilde{M} \rightarrow N$, and also showed, that the space of Whitney jets is isomorphic to the space

$$
C^{\infty}(M, N)=\left\{\left.f\right|_{M}: f \in C^{\infty}(\tilde{M}, N), \tilde{M} \supset M\right\} .
$$

Note that the neighborhood $\tilde{M}$ can be chosen independently of the mapping $f$, but dependent on $N$. This describes a nonlinear extension operator $C^{\infty}(M, N) \rightarrow C^{\infty}(\tilde{M}, N)$; we shall see in section 5 that this extension operator is continuous and even smooth in the manifold structures.

For finite $n$ we shall need the space $C^{\infty, n}\left(\mathbb{R} \times M, \mathbb{R}^{p}\right)$ of restrictions to $M$ of mappings $\mathbb{R} \times \tilde{M} \ni(t, x) \mapsto f(t, x) \in \mathbb{R}^{p}$ which are $C^{\infty}$ in $t$ and $C^{n}$ in $x$. If $\tilde{M}$ is open in $\mathbb{R}^{m}$ we mean by this that any partial derivative $\partial_{t}^{k} \partial_{x}^{\alpha} f$ of any order $k \in \mathbb{N}_{\geq 0}$ in $t$ and of order $|\alpha| \leq n$ in $x$ exists and is continuous on $\mathbb{R} \times \tilde{M}$. This carries over to an open manifold $\tilde{M}$, and finally, using again a tubular neighborhood $p: U \rightarrow i(N)$ as above, to the space $C^{\infty, n}(\mathbb{R} \times M, N)$, for any open manifold $N$. For a treatment of $C^{m, n}$-maps leading to an exponential law see 2]; since $C^{n}$ is not accessible to a convenient approach, a more traditional calculus has to be used there.

By a (vector or fiber) bundle $E \rightarrow M$ over a germ of a Whitney manifold $M$ we mean the restriction to $M$ of a (vector or fiber) bundle $\tilde{E} \rightarrow \tilde{M}$, i.e., of a (vector or fiber) bundle over an open manifold neighborhood. By a smooth section of $E \rightarrow M$ we mean the restriction of a smooth section of $\tilde{E} \rightarrow \tilde{M}$ for a neighborhood $\tilde{M}$. Using classifying smooth mappings into a suitable Grassmannian for vector bundles over $M$ and using the discussion above one could talk about Whitney jets of vector bundles and extend them to a manifold neighborhood of $M$.

We shall use the following spaces of sections of a vector bundle $E \rightarrow M$ over a Whitney manifold germ $M$. This is more general than [55, Section 30], since we allow Whitney manifold germs as base.

- $\Gamma(E)=\Gamma(M \leftarrow E)$, the space of smooth sections, i.e., restrictions of smooth sections of $\tilde{E} \rightarrow \tilde{M}$ for a fixed neighborhood $\tilde{M}$, with the Fréchet space topology of compact convergence on the isomorphic space of Whitney jets of sections.

- $\Gamma_{c}(E)$, the space of smooth sections with compact support, with the inductive limit (LF)-topology.

- $\Gamma_{C^{n}}(E)$, the space of $C^{n}$-section, with the Fréchet space topology of compact convergence on the space of Whitney $n$-jets. If $M$ is compact and $n$ finite, $\Gamma_{C^{n}}(E)$ is a Banach space.

- $\Gamma_{H^{s}}(E)$, the space of Sobolev $H^{s}$-sections, for $0 \leq s \in \mathbb{R}$. Here $M$ should be a compact Whitney manifold germ. The measure on $M$ is the restriction of the volume density with respect to a Riemannian metric on $\tilde{M}$. One also needs a fiber metric on $E$. The space $\Gamma_{H^{k}}(E)$ is independent of all choices, but the inner product depends on the choices. One way to define $\Gamma_{H^{k}}(E)$ is to use a finite atlas which trivializes $\left.\tilde{E}\right|_{L}$ over a compact manifold with smooth boundary $L$ which is a neighborhood of $M$ in $\tilde{M}$ and a partition of unity, and 
then use the Fourier transform description of the Sobolev space. For a careful description see [7. For $0 \leq k<s-\operatorname{dim}(M) / 2$ we have $\Gamma_{H^{s}}(E) \subset \Gamma_{C^{k}}(E)$ continuously.

- More generally, for $0 \geq s \in \mathbb{R}$ and $1<p<\infty$ we also consider $\Gamma_{W^{s, p}}(E)$, the space of $W^{s, p}$-sections: For integral $s$, all (covariant) derivatives up to order $s$ are in $L^{p}$. For $0 \leq k<s-\operatorname{dim}(M) / p$ we have $\Gamma_{H^{s}}(E) \subset \Gamma_{C^{k}}(E)$ continuously.

4.9. Is Stokes' theorem valid for Whitney manifold germs? This seems far from obvious. Here is an example, due to [43]:

By the first answer to the MathOverflow question [50] there is a set $K$ in $[0,1] \subset \mathbb{R}$ which is the closure of its open interior such that the boundary is a Cantor set with positive Lebesgue measure. Moreover, $\mathbb{R} \supset K$ is a Whitney pair by [102, or by the local Markov inequalities [40, Proposition 4.8], or by 41. To make this connected, consider $K_{2}:=(K \times[0,2]) \cup([0,1] \times[1,2])$ in $\mathbb{R}^{2}$. Then $\mathbb{R}^{2} \supset K_{2}$ is again a Whitney pair, but $\partial K_{2}$ has positive 2 -dimensional Lebesgue measure.

As an aside we remark that Cantor-like closed sets in $\mathbb{R}$ might or might not admit continuous extension operators; see [101, Beispiel 1], [102, and the final result in [5], where a complete characterisation is given in terms of the logarithmic dimension of the Cantor-like set.

4.10. Theorem. [52, Theorem 4] Let $M$ be a connected compact oriented Whitney manifold germ. Let $\omega_{0}, \omega \in \Omega^{m}(M)$ be two volume forms (both $>0$ ) with $\int_{M} \omega_{0}=\int_{M} \omega$. Suppose that there is a diffeomorphism $f: M \rightarrow M$ such that $\left.f^{*} \omega\right|_{U}=\left.\omega_{0}\right|_{U}$ for an open neighborhood of $\partial M$ in $M$.

Then there exists a diffeomorphism $\tilde{f}: M \rightarrow M$ with $\tilde{f}^{*} \omega=\omega_{0}$ such that $\tilde{f}$ equals $f$ on an open neighborhood of $\partial M$.

This relative Moser theorem for Whitney manifold germs is modeled on the standard proof of Moser's theorem in [73, Theorem 31.13]. The proof of [52, Theorem 4] is for manifolds with corners, but it works without change for Whitney manifold germs.

\section{MANIFOLDS OF MAPPINGS}

In this section we demonstrate how convenient calculus allows for very short and transparent proofs of the core results in the theory of manifolds of smooth mappings. We follow [55] but we allow the source manifold to be a Whitney manifold germ. In 69 $M$ was allowed to have corners. We will treat manifolds of smooth mappings, and of $C^{n}$-mappings, and we will also mention the case of Sobolev mappings.

5.1. Lemma (Smooth curves into spaces of sections of vector bundles). Let $p: E \rightarrow M$ be a vector bundle over a compact smooth manifold $M$, possibly with corners.

(1) Then the space $C^{\infty}(\mathbb{R}, \Gamma(E))$ of all smooth curves in $\Gamma(E)$ consists of all $c \in C^{\infty}(\mathbb{R} \times M, E)$ with $p \circ c=\operatorname{pr}_{2}: \mathbb{R} \times M \rightarrow M$. 
(2) Then the space $C^{\infty}\left(\mathbb{R}, \Gamma_{C^{n}}(E)\right)$ of all smooth curves in $\Gamma_{C^{n}}(E)$ consists of all $c \in C^{\infty, n}(\mathbb{R} \times M, E)$ (see 4.8) with $p \circ c=\operatorname{pr}_{2}: \mathbb{R} \times M \rightarrow M$.

(3) If $M$ is a compact manifold or a compact Whitney manifold germ, then for each $1<p<\infty$ and $s \in(\operatorname{dim}(M) / p, \infty)$ the space $C^{\infty}\left(\mathbb{R}, \Gamma_{W^{s, p}}(E)\right)$ of smooth curves in $\Gamma_{W^{s, p}}(E)$ consists of all continuous mappings $c: \mathbb{R} \times M \rightarrow E$ with $p \circ c=\operatorname{pr}_{2}: \mathbb{R} \times M \rightarrow M$ such that the following two conditions hold:

- For each $x \in M$ the curve $t \mapsto c(t, x) \in E_{x}$ is smooth; let $\left(\partial_{t}^{k} c\right)(t, x)=\partial_{t}^{k}(c(, x))(t)$.

- For each $k \in \mathbb{N}_{\geq 0}$, the curve $\partial_{t}^{k} c$ has values in $\Gamma_{W^{s, p}}(E)$ so that $\partial_{t}^{k} c: \mathbb{R} \rightarrow \Gamma_{W^{s, p}}(E)$, and $t \mapsto\left\|\partial_{t}^{k} c(t, \cdot)\right\|_{\Gamma_{W^{s}, p}(E)}$ is bounded, locally in $t$.

(4) If $M$ is an open manifold, then the space $C^{\infty}\left(\mathbb{R}, \Gamma_{c}(E)\right)$ of all smooth curves in the space $\Gamma_{c}(E)$ of smooth sections with compact support consists of all $c \in C^{\infty}(\mathbb{R} \times M, E)$ with $p \circ c=\operatorname{pr}_{2}: \mathbb{R} \times M \rightarrow M$ such that

- for each compact interval $[a, b] \subset \mathbb{R}$ there is a compact subset $K \subset M$ such that $c(t, x)=0$ for $(t, x) \in[a, b] \times(M \backslash K)$.

Likewise for the space $C^{\infty}\left(\mathbb{R}, \Gamma_{C^{n}, c}(E)\right)$ of smooth curves in the space of $C^{n}$ sections with compact support.

(5) Let $p: E \rightarrow M$ be a vector bundle over a compact Whitney manifold germ. Then the space $C^{\infty}(\mathbb{R}, \Gamma(E))$ of smooth curves in $\Gamma(E)$ consists of all smooth mappings $c: \mathbb{R} \times \tilde{M} \rightarrow \tilde{E}$ with $p \circ c=\operatorname{pr}_{2}: \mathbb{R} \times \tilde{M} \rightarrow \tilde{M}$ for some open neighborhood manifold $\tilde{M}$ and extended vector bundle $\tilde{E}$. We may even assume that there is a compact submanifold with smooth boundary $L \subset \tilde{M}$ containing $M$ in its interior such that $c(t, x)=0$ for $(t, x) \in \mathbb{R} \times(\tilde{M} \backslash L)$. Using the last statement of 4.1 , this is equivalent to the space of all smooth mappings $c: \mathbb{R} \times M \rightarrow E \subset \bar{E}$ with $p \circ c=\operatorname{pr}_{2}: \mathbb{R} \times M \rightarrow M$.

(6) Let $p: E \rightarrow M$ be a vector bundle over a non-compact Whitney manifold germ $M \subset \tilde{M}$, then the space $C^{\infty}\left(\mathbb{R}, \Gamma_{c}(E)\right)$ of all smooth curves in the space

$$
\Gamma_{c}(E)=\left\{\left.s\right|_{M}: s \in \Gamma_{c}(\tilde{M} \leftarrow \tilde{E})\right\}
$$

of smooth sections with compact support (see 4.8) consists of all smooth mappings $c: \mathbb{R} \times \tilde{M} \rightarrow \tilde{E}$ with $p \circ c=\operatorname{pr}_{2}: \mathbb{R} \times M \rightarrow \tilde{M}$ such that

- for each compact interval $[a, b] \subset \mathbb{R}$ there is a compact subset $K \subset \tilde{M}$ such that $c(t, x)=0$ for $(t, x) \in[a, b] \times(M \backslash K)$.

Proof. (1) This follows from the exponential law 2.46 after trivializing the bundle.

(2) We trivialize the bundle, assume that $M$ is open in $\mathbb{R}^{m}$, and then prove this directly. In [55, 3.1 and 3.2] one finds a very explicit proof of the case $n=\infty$, which one can restrict to our case here.

(3) To see this we first choose a second vector bundle $F \rightarrow M$ such that $E \oplus_{M} F$ is a trivial bundle, i.e., isomorphic to $M \times \mathbb{R}^{n}$ for some $n \in \mathbb{N}$. Then $\Gamma_{W^{s, p}}(E)$ is a direct summand in $W^{s, p}\left(M, \mathbb{R}^{n}\right)$, so that we may assume without loss that $E$ is a trivial bundle, and then, that it is 1-dimensional. So we have to identify $C^{\infty}\left(\mathbb{R}, W^{s, p}(M, \mathbb{R})\right)$. But in this situation we can 
just apply Theorem 2.6 for the set $\mathcal{V} \subset W^{s, p}(M, \mathbb{R})^{\prime}$ consisting of all point evaluations $\operatorname{ev}_{x}: H^{s}(M, \mathbb{R}) \rightarrow \mathbb{R}$ and use that $W^{s, p}(M, \mathbb{R})$ is a reflexive Banach space.

(4) This is like (1) or (2) where we have to assure that the curve $c$ takes values in the space of sections with compact support which translates to the condition.

(5) and (6) follow from (4) after extending to $\tilde{E} \rightarrow \tilde{M}$.

5.2. Lemma. Let $E_{1}, E_{2}$ be vector bundles over smooth manifold or a Whitney manifold germ $M$, let $U \subset E_{1}$ be an open neighborhood of the image of a smooth section, let $F: U \rightarrow E_{2}$ be a fiber preserving smooth mapping. Then the following statements hold:

(1) If $M$ is compact, the set $\Gamma(U):=\left\{h \in \Gamma\left(E_{1}\right): h(M) \subset U\right\}$ is open in $\Gamma\left(E_{1}\right)$, and the mapping $F_{*}: \Gamma(U) \rightarrow \Gamma\left(E_{2}\right)$ given by $h \mapsto F \circ h$ is smooth. Likewise for spaces $\Gamma_{c}\left(E_{i}\right)$, if $M$ is not compact.

(2) If $M$ is compact, for $n \in \mathbb{N}_{\geq 0}$ the set

$$
\Gamma_{C^{n}}(U):=\left\{h \in \Gamma_{C^{n}}\left(E_{1}\right): h(M) \subset U\right\}
$$

is open in $\Gamma_{C^{n}}\left(E_{1}\right)$, and the mapping $F_{*}: \Gamma_{C^{n}}(U) \rightarrow \Gamma_{C^{n}}\left(E_{2}\right)$ given by $h \mapsto F \circ h$ is smooth.

(3) If $M$ is compact and $s>\operatorname{dim}(M) / p$, the set

$$
\Gamma_{W^{s, p}}(U):=\left\{h \in \Gamma_{W^{s, p}}\left(E_{1}\right): h(M) \subset U\right\}
$$

is open in $\Gamma_{W^{s, p}}\left(E_{1}\right)$, and the mapping $F_{*}: \Gamma_{W^{s, p}}(U) \rightarrow \Gamma_{W^{s, p}}\left(E_{2}\right)$ given by $h \mapsto F \circ h$, is smooth.

If the restriction of $F$ to each fiber of $E_{1}$ is real analytic, then $F_{*}$ is real analytic; but in this paper we concentrate on $C^{\infty}$ only. This lemma is a variant of the so-called Omega-lemma; e.g., see [69]. Note how simple the proof is using convenient calculus.

Proof. Without loss suppose that $U=E_{1}$.

(1) and (2) follow easily since $F_{*}$ maps smooth curves to smooth curves; see their description in (5.1 1) and (5.12).

(3) Let $c: \mathbb{R} \ni t \mapsto c(t,) \in \Gamma_{W^{s, p}\left(E_{1}\right)}$ be a smooth curve. As $s>\operatorname{dim}(M) / 2$, it holds for each $x \in M$ that the mapping $\mathbb{R} \ni t \mapsto F_{x}(c(t, x)) \in\left(E_{2}\right)_{x}$ is smooth. By the Faà di Bruno formula (see [34] for the 1-dimensional version, preceded in 3 by 55 years), we have for each $p \in \mathbb{N}_{>0}, t \in \mathbb{R}$, and $x \in M$ that

$$
\begin{aligned}
& \partial_{t}^{p} F_{x}(c(t, x))= \\
& \quad=\sum_{j \in \mathbb{N}_{>0}} \sum_{\substack{\alpha \in \mathbb{N}_{>0}^{j} \\
\alpha_{1}+\cdots+\alpha_{j}=p}} \frac{1}{j !} d^{j}\left(F_{x}\right)(c(t, x))\left(\frac{\partial_{t}^{\left(\alpha_{1}\right)} c(t, x)}{\alpha_{1} !}, \ldots, \frac{\partial_{t}^{\left(\alpha_{j}\right)} c(t, x)}{\alpha_{j} !}\right) .
\end{aligned}
$$

For each $x \in M$ and $\alpha_{x} \in\left(E_{2}\right)_{x}^{*}$ the mapping $s \mapsto\left\langle s(x), \alpha_{x}\right\rangle$ is a continuous linear functional on the Hilbert space $\Gamma_{W^{s, p}}\left(E_{2}\right)$. The set $\mathcal{V}_{2}$ of all 
of these functionals separates points and therefore satisfies the condition of Theorem 2.6. We also have for each $p \in \mathbb{N}_{>0}, t \in \mathbb{R}$, and $x \in M$ that

$$
\partial_{t}^{p}\left\langle F_{x}(c(t, x)), \alpha_{x}\right\rangle=\left\langle\partial_{t}^{p} F_{x}(c(t, x)), \alpha_{x}\right\rangle=\left\langle\partial_{t}^{p} F_{x}(c(t, x)), \alpha_{x}\right\rangle .
$$

Using the explicit expressions for $\partial_{t}^{p} F_{x}(c(t, x))$ from above we may apply Lemma 5.1.3) to conclude that $t \mapsto F(c(t)$,$) is a smooth curve \mathbb{R} \rightarrow$ $\Gamma_{H^{s}}\left(E_{1}\right)$. Thus, $F_{*}$ is a smooth mapping.

5.3. The manifold structure on $C^{\infty}(M, N)$ and $C^{k}(M, N)$. Let $M$ be a compact or open finite dimensional smooth manifold or even a compact Whitney manifold germ, and let $N$ be a smooth manifold. We use an auxiliary Riemannian metric $\bar{g}$ on $N$ and its exponential mapping $\exp ^{\bar{g}}$; some of its properties are summarized in the following diagram:

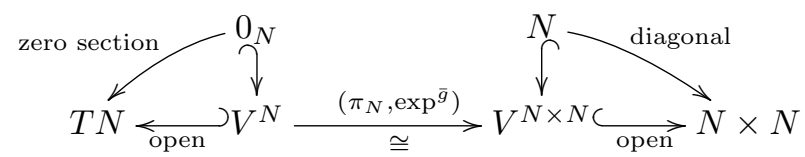

Without loss we may assume that $V^{N \times N}$ is symmetric:

$$
\left(y_{1}, y_{2}\right) \in V^{N \times N} \Longleftrightarrow\left(y_{2}, y_{1}\right) \in V^{N \times N} .
$$

- If $M$ is compact, then $C^{\infty}(M, N)$, the space of smooth mappings $M \rightarrow N$, has the following manifold structure. A chart, centered at $f \in C^{\infty}(M, N)$, is:

$$
\begin{gathered}
C^{\infty}(M, N) \supset U_{f}=\left\{g:(f, g)(M) \subset V^{N \times N}\right\} \stackrel{u_{f}}{\longrightarrow} \tilde{U}_{f} \subset \Gamma\left(M \leftarrow f^{*} T N\right) \\
u_{f}(g)=\left(\pi_{N}, \exp ^{\bar{g}}\right)^{-1} \circ(f, g), \quad u_{f}(g)(x)=\left(\exp _{f(x)}^{\bar{g}}\right)^{-1}(g(x)) \\
\left(u_{f}\right)^{-1}(s)=\exp _{f}^{\bar{g}} \circ s, \quad\left(u_{f}\right)^{-1}(s)(x)=\exp _{f(x)}^{\bar{g}}(s(x))
\end{gathered}
$$

Note that $\tilde{U}_{f}$ is open in $\Gamma\left(M \leftarrow f^{*} T N\right)$ if $M$ is compact.

- If $M$ is open, then the compact $C^{\infty}$-topology on $\Gamma\left(f^{*} T N\right)$ is not suitable since $\tilde{U}_{f}$ is in general not open. We have to control the behavior of sections near infinity on $M$. One solution is to use the space $\Gamma_{c}\left(f^{*} T N\right)$ of sections with compact support as modeling spaces and to adapt the topology on $C^{\infty}(M, N)$ accordingly. This has been worked out in [69] and [55].

- If $M$ is compact Whitney manifold germ with neighborhood manifold $\tilde{M} \supset$ $M$ we use the Fréchet space $\Gamma\left(M \leftarrow f^{*} T N\right)=\left\{\left.s\right|_{M}: s \in \Gamma_{L}\left(\tilde{M} \leftarrow \tilde{f}^{*} T N\right)\right\}$ where $L \subset \tilde{M}$ is a compact set containing $M$ in its interior and $\tilde{f}: \tilde{M} \rightarrow N$ is an extension of $f$ to a suitable manifold neighborhood of $M$. Via an extension operator the Fréchet space $\Gamma\left(M \leftarrow f^{*} T N\right)$ is a direct summand in the Fréchet space $\Gamma_{L}\left(\tilde{M} \leftarrow \tilde{f}^{*} T N\right)$ of smooth sections with support in $L$.

- Likewise, for a non-compact Whitney manifold germ we use the convenient (LF)-space

$$
\Gamma_{c}\left(M \leftarrow f^{*} T N\right)=\left\{\left.s\right|_{M}: s \in \Gamma_{c}\left(\tilde{M} \leftarrow \tilde{f}^{*} T N\right)\right\}
$$

of sections with compact support. 
- On the space $C^{k}(M, N$,$) for k \in \mathbb{N}_{\geq 0}$ we use only charts as described above with the center $f \in C^{\infty}(M, N)$, namely

$$
C^{k}(M, N) \supset U_{f}=\left\{g:(f, g)(M) \subset V^{N \times N}\right\} \stackrel{u_{f}}{\longrightarrow} \tilde{U}_{f} \subset \Gamma_{C^{k}}\left(M \leftarrow f^{*} T N\right) .
$$

We claim that these charts cover $C^{k}(M, N)$ : Since $C^{\infty}(M, N)$ is dense in $C^{k}(M, N)$ in the Whitney $C^{k}$-topology, for any $g \in C^{k}(M, N)$ there exists $f \in C^{\infty}(M, N,) \cap U_{g}$. But then $g \in U_{f}$ since $V^{N \times N}$ is symmetric. This is true for compact $M$. For a compact Whitney manifold germ we can apply this argument in a compact neighborhood $L$ of $M$ in $\tilde{M}$, replacing $\tilde{M}$ by the interior of $L$ after the fact.

- On the space $W^{s, p}(M, N)$ for $\operatorname{dim}(M) / p<s \in \mathbb{R}$ we use only charts as described above with the center $f \in C^{\infty}(M, N)$, namely:

$$
\begin{aligned}
W^{s, p}(M, N) \supset U_{f}=\left\{g:(f, g)(M) \subset V^{N \times N}\right\} \stackrel{u_{f}}{\longrightarrow} \\
\stackrel{u_{f}}{\longrightarrow} \tilde{U}_{f} \subset \Gamma_{W^{s, p}}\left(M \leftarrow f^{*} T N\right) .
\end{aligned}
$$

These charts cover $W^{s, p}(M, N)$, by the following argument: Since $C^{\infty}(M, N)$ is dense in $W^{s, p}(M, N)$ and since $W^{s, p}(M, N) \subset C^{k}(M, N)$ via a continuous injection for $0 \leq k<s-\operatorname{dim}(M) / p$, a suitable $C^{0}-$ sup-norm neighborhood of $g \in W^{s, p}(M, N)$ contains a smooth $f \in C^{\infty}(M, N)$, thus $f \in U_{g}$ and by symmetry of $V^{N \times N}$ we have $g \in U_{f}$. This is true for compact $M$. For a compact Whitney manifold germ we can apply this argument in a compact neighborhood which is a manifold with smooth boundary $L$ of $M$ in $\tilde{M}$ and apply the argument there.

In each case, we equip $C^{\infty}(M, N)$ or $C^{k}(M, N)$ or $W^{s, p}(M, N)$ with the initial topology with respect to all chart mappings described above: The coarsest topology, so that all chart mappings $u_{f}$ are continuous.

For non-compact $M$ the direct limit $\Gamma_{c}\left(f^{*} T N\right)=\lim _{L} \Gamma_{L}\left(f^{*} T N\right)$ over a compact exhaustion $L$ of $M$ in the category of locally convex vector spaces is strictly coarser that the direct limit in the category of Hausdorff topological spaces. It is more convenient to use the latter topology which is called $c^{\infty}$ topology; compare with 2.1 .

5.4. Lemma. (1) If $M$ is a compact smooth manifold or is a compact Whitney manifold germ,

$$
C^{\infty}\left(\mathbb{R}, \Gamma\left(M \leftarrow f^{*} T N\right)\right)=\Gamma\left(\mathbb{R} \times M \leftarrow \operatorname{pr}_{2}{ }^{*} f^{*} T N\right) .
$$

For smooth $f \in C^{\infty}(M, N)$,

$$
C^{\infty}\left(\mathbb{R}, \Gamma_{C^{n}}\left(M \leftarrow f^{*} T N\right)\right)=\Gamma_{C^{\infty, n}}\left(\mathbb{R} \times M \leftarrow \operatorname{pr}_{2}{ }^{*} f^{*} T N\right) .
$$

(2) If $M$ is a non-compact smooth manifold of Whitney manifold germ, the sections on the right hand-side have to satisfy the corresponding conditions of lemma 5.1.4).

For a compact Whitney manifold germ $M$ the space $\Gamma\left(\mathbb{R} \times M \leftarrow \operatorname{pr}_{2}{ }^{*} f^{*} T N\right)$ is a direct summand in the space $\Gamma_{\mathbb{R} \times L}\left(\mathbb{R} \times \tilde{M} \leftarrow \mathrm{pr}_{2}{ }^{*} f^{*} T N\right)$ of sections 
with support in $\mathbb{R} \times L$ for a fixed compact set $L \subset \tilde{M}$ containing $M$ in its interior. Likewise $\Gamma_{C \infty, n}\left(\mathbb{R} \times M \leftarrow \operatorname{pr}_{2}{ }^{*} f^{*} T N\right)$ is a direct summand in the space $\Gamma_{C^{\infty, n}, \mathbb{R} \times L}\left(\mathbb{R} \times \tilde{M} \leftarrow \operatorname{pr}_{2}{ }^{*} f^{*} T N\right)$ of $C^{\infty, n}$-sections. One could introduce similar notation for $C^{\infty}\left(\mathbb{R}, \Gamma_{W^{s, p}}\left(M \leftarrow f^{*} T N\right)\right)$.

Proof. This follows from lemma 5.1 .

5.5. Lemma. Let $M$ be a smooth manifold or Whitney manifold germ, compact or not, and let $N$ be a manifold. Then the chart changes for charts centered on smooth mappings are smooth $\left(C^{\infty}\right)$ on the space $C^{\infty}(M, N)$, also on $C^{k}(M, N)$ for $k \in \mathbb{N}_{\geq 0}$, and on $W^{s, p}(M, N)$ for $1<p<\infty$ and $s>\operatorname{dim}(M) / p$ :

$$
\tilde{U}_{f_{1}} \ni s \mapsto\left(u_{f_{2}, f_{1}}\right)_{*}(s):=\left(\exp _{f_{2}}^{\bar{g}}\right)^{-1} \circ \exp _{f_{1}}^{\bar{g}} \circ s \in \tilde{U}_{f_{2}} .
$$

Proof. This follows from Lemma 5.2, since any chart change is just compositions from the left by a smooth fiber respecting locally defined diffeomorphism.

5.6. Lemma. (1) If $M$ is a compact manifold or a compact Whitney manifold germ, then

$$
C^{\infty}\left(\mathbb{R}, C^{\infty}(M, N)\right) \cong C^{\infty}(\mathbb{R} \times M, N) .
$$

(2) If $M$ is not compact, $C^{\infty}\left(\mathbb{R}, C^{\infty}(M, N)\right)$ consists of all smooth $c: \mathbb{R} \times$ $M \rightarrow N$ such that

- for each compact interval $[a, b] \subset \mathbb{R}$ there is a compact subset $K \subset M$ such that $c(t, x)$ is constant in $t \in[a, b]$ for each $x \in M \backslash K$.

Proof. By lemma 5.4 .

5.7. Lemma. Composition $(f, g) \mapsto g \circ f$ is smooth as a mapping

$$
\begin{aligned}
C^{\infty}(P, M) & \times C^{\infty}(M, N) \rightarrow C^{\infty}(P, N) \\
C^{k}(P, M) & \times C^{\infty}(M, N) \rightarrow C^{k}(P, N) \\
W^{s, p}(P, M) & \times C^{\infty}(M, N) \rightarrow W^{s, p}(P, N)
\end{aligned}
$$

for $P$ a manifold or a Whitney manifold germ, compact or not, and for $M$ and $N$ manifolds.

For more general $M$ the description becomes more complicated. See the special case of the diffeomorphism group of a Whitney manifold germ $M$ in 6.3 below.

Proof. Since it maps smooth curves to smooth curves.

5.8. Corollary. For $M$ a manifold or a Whitney manifold germ and a manifold $N$, the tangent bundle of the manifold $C^{\infty}(M, N)$ of mappings is given by

$$
T C^{\infty}(M, N)=C^{\infty}(M, T N) \stackrel{C^{\infty}\left(M, \pi_{N}\right)=\left(\pi_{N}\right)_{*}}{\longrightarrow} C^{\infty}(M, N),
$$




$$
\begin{aligned}
T C^{k}(M, N) & =C^{k}(M, T N) \stackrel{C^{k}\left(M, \pi_{N}\right)=\left(\pi_{N}\right)_{*}}{\longrightarrow} C^{k}(M, N), \\
T W^{s, p}(M, N) & =W^{s, p}(M, T N) \stackrel{W^{s, p}\left(M, \pi_{N}\right)=\left(\pi_{N}\right)_{*}}{\longrightarrow} W^{s, p}(M, N) .
\end{aligned}
$$

Proof. This follows from the chart structure and the fact that sections of $f^{*} T N \rightarrow M$ correspond to mappings $s: M \rightarrow T N$ with $\pi_{N} \circ s=f$.

5.9. Sprays respecting fibers of submersions. Sprays are versions of Christoffel symbols and lead to exponential mappings. They are easier to adapt to fibered manifolds than Riemannian metrics. Recall that a spray $S$ on a manifold $N$ without boundary is a smooth mapping $S: T N \rightarrow T^{2} N$ with the following properties:

- $\pi_{T N} \circ S=\operatorname{Id}_{T N} ; S$ is a vector field.

- $T\left(\pi_{N}\right) \circ S=\operatorname{Id}_{T N} ; S$ is a 'differential equation of second order'.

- Let $m_{t}^{N}: T N \rightarrow T N$ and $m_{t}^{T N}: T^{2} N \rightarrow T^{2} N$ be the scalar multiplications. Then $S \circ m_{t}^{N}=T\left(m_{t}^{N}\right) \cdot m_{t}^{T N} \cdot S$.

Locally, in charts of $T N$ and $T^{2} N$ induced by a chart of $N$, a spray looks like $S(x, v)=(x, v ; v ; \Gamma(x, v))$ where $\Gamma$ is quadratic in $v$. For a spray $S \in$ $\mathfrak{X}(T N)$ on a manifold $N$, we let $\exp (X):=\pi_{N}\left(\mathrm{Fl}_{1}^{S}(X)\right)$, then the mapping $\exp : T N \supset V \rightarrow N$ is smooth, defined on an open neighborhood $V$ of the zero section in $T N$, which is called the exponential mapping of the spray $S$. Since $T_{0_{x}}\left(\left.\exp \right|_{T_{x} N}\right)=\operatorname{Id}_{T_{x} N}\left(\right.$ via $\left.T_{0_{x}}\left(T_{x} N\right)=T_{x} N\right)$, by the inverse function theorem $\exp _{x}:=\left.\exp \right|_{T_{x} N}$ is a diffeomorphism near $0_{x}$ in $T N$ onto an open neighborhood of $x$ in $N$. Moreover the mapping $\left(\pi_{N}, \exp \right): T N \supset \tilde{V} \rightarrow$ $N \times N$ is a diffeomorphism from an open neighborhood $\tilde{V}$ of the zero section in $T N$ onto an open neighborhood of the diagonal in $N \times N$.

Lemma. Let $q: N \rightarrow M$ be a smooth surjective submersion between connected manifolds without boundary. Then there exists a spray $S$ on $N$ which is tangential to the fibers of $q$, i.e., $S\left(T\left(q^{-1}(x)\right)\right) \subset T^{2}\left(q^{-1}(x)\right)$ for each $x \in M$.

This is a simplified version of [69, 10.9].

Proof. In suitable charts on $N$ and $M$ the submersion $q$ looks like a linear projection $\left(y_{1}, y_{2}\right) \mapsto y_{1}$. The local expression $T$ (chart) $\rightarrow T^{2}$ (chart) of a spray is

$$
\begin{aligned}
& S\left(\left(y_{1}, y_{2}\right),\left(v_{1}, v_{2}\right)\right)= \\
& =\left(\left(y_{1}, y_{2}\right),\left(v_{1}, v_{2}\right) ;\left(v_{1}, v_{2}\right),\left(\Gamma^{1}\left(y_{1}, y_{2} ; v_{1}, v_{2}\right), \Gamma^{2}\left(y_{1}, y_{2}, v_{1}, v_{2}\right)\right)\right)
\end{aligned}
$$

where $\Gamma^{i}\left(y_{1}, y_{2}, v_{1}, v_{2}\right)$ is quadratic in $\left(v_{1}, v_{2}\right)$. The spray is tangential to the fibers of $q$ if and only if $\Gamma^{1}\left(y_{1}, y_{2}, 0, v_{2}\right)=0$. This clearly exists locally (e.g., choose $\left.\Gamma^{1}=0\right)$. Now we use a partition of unity $\left(\varphi_{\alpha}\right)$ subordinated to a cover $N=\bigcup_{\alpha} U_{\alpha}$ with such charts and glue local sprays with the induced partition of unity $\left(\varphi_{\alpha} \circ \pi_{N}\right)$ subordinated to the cover $T N=\bigcup_{\alpha} T U \alpha$ for the vector 
bundle $\pi_{T N}: T^{2} N \rightarrow T N$. Locally this looks like (where $y=\left(y_{1}, y_{2}\right)$ etc.)

$$
\begin{aligned}
\left(\sum_{\alpha}\left(\varphi_{\alpha} \circ \pi_{N}\right) . S_{\alpha}\right)(y, v) & =\left(y, v ; \sum_{\alpha} \varphi_{\alpha}(y) v, \sum_{\alpha} \varphi_{\alpha}(y)\left(\Gamma_{\alpha}^{1}(y, v),\left(\Gamma_{\alpha}^{2}(y, v)\right)\right)\right. \\
& =\left(y, v ; v,\left(\sum_{\alpha} \varphi_{\alpha}(y) \Gamma_{\alpha}^{1}(y, v), \sum_{\alpha} \varphi_{\alpha}(y) \Gamma_{\alpha}^{2}(y, v)\right)\right)
\end{aligned}
$$

and is therefore a spray which is tangential to the fibers of $q$.

5.10. Proposition. [69, 10.10] Let $q: N \rightarrow M$ be a smooth surjective submersion between connected manifolds without boundary. The space $S^{q}(M, N)$ of all smooth sections of $q$ is a splitting smooth submanifold of $C^{\infty}(M, N)$. Similarly, the spaces $S_{C^{N}}^{q}(M, N)$ and $S_{W^{s, p}}^{q}(M, N)$ of $C^{N_{-}}$-sections and $W^{s, p}$ sections are smooth splitting submanifolds of $C^{N}(M, N)$ or $W^{s, p}(M, N)$ (for $s>\operatorname{dim}(M) / p)$, respectively.

The proof given here is simpler than the one in [69, 10.10].

Proof. Let us first assume that $M$ is compact. Given a smooth section $f \in$ $S^{q}(M, N)$, consider the chart centered at $f$ from 5.3

$$
\begin{gathered}
C^{\infty}(M, N) \supset U_{f}=\left\{g:(f, g)(M) \subset V^{N \times N}\right\} \stackrel{u_{f}}{\longrightarrow} \tilde{U}_{f} \subset \Gamma\left(M \leftarrow f^{*} T N\right) \\
u_{f}(g)=\left(\pi_{N}, \exp ^{S}\right)^{-1} \circ(f, g), \quad u_{f}(g)(x)=\left(\exp _{f(x)}^{S}\right)^{-1}(g(x)) \\
\left(u_{f}\right)^{-1}(s)=\exp _{f}^{S} \circ s, \quad\left(u_{f}\right)^{-1}(s)(x)=\exp _{f(x)}^{S}(s(x))
\end{gathered}
$$

where we use the exponential mapping with respect to a spray $S$ on $N$ which is tangential to the fibers of $q$. Using an unrelated auxiliary Riemannian metric $\bar{g}$ on $N$ we can smoothly split the tangent bundle $T N=V^{q}(N) \oplus H^{q}(N)$ into the vertical bundle of all vectors tangent to the fibers of $q$, and into its orthogonal complement with respect to $\bar{g}$. The orthonormal projections $P^{\bar{g}}: T N \rightarrow V^{q}(N)$ and $\operatorname{Id}_{T N}-P^{\bar{g}}: T N \rightarrow H^{q}(N)$ induce the direct sum decomposition

$\Gamma\left(M \leftarrow f^{*} T N\right)=\Gamma\left(M \leftarrow f^{*} V^{q}(N)\right) \oplus \Gamma\left(M \leftarrow f^{*} T N\right) \quad s \mapsto\left(P^{\bar{g}} . s, s-P^{\bar{g}} . s\right)$. Now $g \in U_{f}$ is in $S^{q}(M, N)$ if and only if $u_{f}(g) \in \Gamma\left(f^{*} V^{q}(N)\right)$.

If $M$ is not compact we may use the spaces of sections with compact

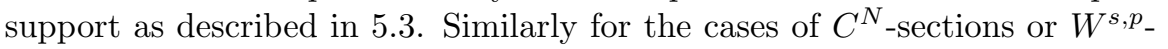
sections.

5.11. Corollary. Let $p: E \rightarrow M$ be a fiber bundle over a compact Whitney manifold germ $M$. Then the space $\Gamma(E)$ of smooth sections is a splitting smooth submanifold of $C^{\infty}(M, E)$. Likewise for the spaces $\Gamma_{C^{N}}(E)$ and $\Gamma_{W^{s, p}}(E)$ of $C^{N}$-sections and $W^{s, p}$-sections.

Proof. Recall from 4.8 that $E=\left.\tilde{E}\right|_{M}$ for a smooth fiber bundle $\tilde{E} \rightarrow \tilde{M}$. There the result follows from 5.10 . Using (fixed) extension operators

$$
\Gamma\left(M \leftarrow f^{*} T E\right) \rightarrow \Gamma_{L}\left(\tilde{M} \leftarrow \tilde{f}^{*} T \tilde{E}\right)
$$

etc. we can extend this the case of Whitney manifold germs. 


\section{Regular Lie Groups}

6.1. Regular Lie groups. We consider a smooth Lie group $G$ with Lie algebra $\mathfrak{g}=T_{e} G$ modeled on convenient vector spaces. The notion of a regular Lie group is originally due to [86, 87, 88, 89, 90, 91] for Fréchet Lie groups, was weakened and made more transparent by [78], and then carried over to convenient Lie groups in [56, see also [55, 38.4]. We shall write $\mu: G \times G \rightarrow G$ for the multiplication with $x . y=\mu(x, y)=\mu_{x}(y)=\mu^{y}(x)$ for left and right translation.

A Lie group $G$ is called regular if the following holds:

- For each smooth curve $X \in C^{\infty}(\mathbb{R}, \mathfrak{g})$ there exists a curve $g \in$ $C^{\infty}(\mathbb{R}, G)$ whose right logarithmic derivative is $X$, i.e.,

$$
\left\{\begin{array}{l}
g(0)=e \\
\partial_{t} g(t)=T_{e}\left(\mu^{g(t)}\right) X(t)=X(t) \cdot g(t)
\end{array}\right.
$$

The curve $g$ is uniquely determined by its initial value $g(0)$, if it exists.

- Put $\operatorname{evol}_{G}^{r}(X)=g(1)$ where $g$ is the unique solution required above. Then $\operatorname{evol}_{G}^{r}: C^{\infty}(\mathbb{R}, \mathfrak{g}) \rightarrow G$ is required to be $C^{\infty}$ also. We have $\operatorname{Evol}_{t}^{X}:=g(t)=\operatorname{evol}_{G}^{r}(t X)$.

Of course we could equivalently use the left logarithmic derivative and the corresponding left evolution operator. Group inversion maps the two concepts into each other. See [55, Section 38] for more information. Up to now, every Lie group modeled on convenient vector spaces is regular.

There are other notions of regularity for infinite dimensional Lie groups: For example, one may require that each curve $X \in L_{\text {loc }}^{1}(\mathbb{R}, \mathfrak{g})$ admits an absolutely continuous curve $\mathrm{Evol}^{X}: \mathbb{R} \rightarrow G$ whose right logarithmic derivative is $X$. See [46] or [49] and references therein. It might be that all these notions of regularity are equivalent for Lie groups modeled on convenient vector spaces.

6.2. Theorem. For each manifold $M$ with or without corners, the diffeomorphism group $\operatorname{Diff}(M)$ is a regular Lie group. Its Lie algebra is the space $\mathfrak{X}(M)$ of all vector fields with the negative of the usual bracket as Lie bracket, if $M$ is compact without boundary. It is the space $\mathfrak{X}_{c}(M)$ of fields with compact support, if $M$ is an open manifold. It is the space $\mathfrak{X}_{\partial}(M)$ of 4.7 of vector fields tangent to the boundary, if $M$ is a compact manifold with corners. If $M$ is not compact with corners, then the Lie algebra is the space $\mathfrak{X}_{c, \partial}(M)$ of boundary respecting vector fields with compact support.

Proof. If $M$ is a manifold without boundary then $\operatorname{Diff}(M) \stackrel{\text { open }}{\longrightarrow} C^{\infty}(M, M)$. If $M$ is open, then the group of diffeomorphisms differing from the identity only on a compact set is open in $\operatorname{Diff}(M)$.

If $M$ has corners we use an open manifold $\tilde{M}$ containing $M$ as a submanifold with corners as in 3.2 . In the description of the chart structure 5.3 for $\operatorname{Diff}(\tilde{M})$ we have to use the exponential mapping for a geodesic spray on $\tilde{M}$ 
such that each component of each $\partial^{q} M$ is totally geodesic. This spray exists; see 3.7 or 5.9 . Restricting all sections to $M$ then yields a smooth chart centered at the identity for $\operatorname{Diff}(M)$. Then we use right translations of this chart. The explicit chart structure on $\operatorname{Diff}(M)$ is described in [69, 10.16]. Extending all sections to $\tilde{M}$ via a fixed continuous linear Whitney extension operator respecting compact support identifies $\operatorname{Diff}(M)$ as a splitting smooth closed submanifold of $\operatorname{Diff}(\tilde{M})$, but not as a subgroup.

Composition is smooth by restricting it from $C^{\infty}(M, M) \times C^{\infty}(M, M)$, using 5.7 and its extension to the situation with corners.

Inversion is smooth: If $t \mapsto f(t, \quad)$ is a smooth curve in $\operatorname{Diff}(M)$, then $f(t, \quad)^{-1}$ satisfies the implicit equation $f\left(t, f(t, \quad)^{-1}(x)\right)=x$, so by the finite dimensional implicit function theorem, $(t, x) \mapsto f(t, \quad)^{-1}(x)$ is smooth. So inversion maps smooth curves to smooth curves, and is smooth.

Let $X(t, x)$ be a time-dependent vector field on $M$ (in $C^{\infty}(\mathbb{R}, \mathfrak{X}(M))$ ). Then $\mathrm{Fl}_{s}^{\partial_{t} \times X}(t, x)=\left(t+s, \mathrm{Evol}^{X}(t, x)\right)$ satisfies the ordinary differential equation

$$
\partial_{t} \operatorname{Evol}(t, x)=X(t, \operatorname{Evol}(t, x)) .
$$

If $X(s, t, x) \in C^{\infty}\left(\mathbb{R}^{2}, \mathfrak{X}(M)\right)$ is a smooth curve of smooth curves in $\mathfrak{X}(M)$, then obviously the solution of the equation depends smoothly also on the further variable $s$, thus evol maps smooth curves of time dependent vector fields to smooth curves of diffeomorphism.

6.3. The diffeomorphism group of a Whitney manifold germ. For a Whitney manifold germ $\tilde{M} \supset M$, we consider the diffeomorphism group

$$
\begin{aligned}
\operatorname{Diff}(M)=\left\{\left.\varphi\right|_{M}: \varphi \in C^{\infty}(\tilde{M}, \tilde{M}), \varphi(M)=M,\right. \\
\varphi \text { is a diffeomorphism on an open neighborhood of } M\} .
\end{aligned}
$$

We also consider the following set $\mathcal{C}$ of smooth curves: Those $c: \mathbb{R} \rightarrow \operatorname{Diff}(M)$ which are of the form $c=\left.\tilde{c}\right|_{\mathbb{R} \times M}$ for a smooth

$$
\tilde{c}: \mathbb{R} \times \tilde{M} \rightarrow \tilde{M} \quad \text { with }\left.\tilde{c}(t,)\right|_{M} \in \operatorname{Diff}(M) \text { for each } t \in \mathbb{R} .
$$

Note that for $t$ in a compact interval $\tilde{c}(t$,$) is a diffeomorphism on a fixed$ open neighborhood of $M$ in $\tilde{M}$.

6.4. Theorem. For a Whitney manifold germ $M$ the group $\operatorname{Diff}(M)$ is a Frölicher space and a group with smooth composition and inversion. It has a convenient Lie algebra $\mathfrak{X}_{c, \partial}(M)$ with the negative of the usual bracket as Lie bracket, and it is regular: There exists an evolution operator and it is smooth.

Proof. The Frölicher space structure is the one induced by the set $\mathcal{C}$ of smooth curves described above. I do not know whether this set of smooth curves is saturated, i.e., $\mathcal{C}=\mathcal{C}_{\operatorname{Diff}(M)}$ in the notation of 2.7 this might depend on the structure of the boundary.

The proof is now quite similar to the one of 6.1. We claim that composition maps $\mathcal{C} \times \mathcal{C}$ to $\mathcal{C} \subseteq \mathcal{C}_{\text {Diff }(M)}$, and that inversion maps $\mathcal{C}$ to $\mathcal{C} \subseteq \mathcal{C}_{\text {Diff }(M)}$. Since 
by definition each curve $c \in \mathcal{C}$ extend to a smooth mapping $\tilde{c}: \mathbb{R} \times \tilde{M} \rightarrow \tilde{M}$ we can actually use a slight adaption of the proof of 6.1 for open manifolds.

6.5. The connected component of $\operatorname{Diff}(M)$ for a Whitney manifold germ $M$. We consider a Whitney manifold germ $M \subset \tilde{M}$. As usual for Frölicher space, we equip $\operatorname{Diff}(M)$ with the final topology with respect to all smooth curves in in the generating set $\mathcal{C}$ as described in 6.3. $\operatorname{Diff}(M)$ is actually a topological group, with the refined topology (i.e., the $c^{\infty}$-topology) on $\operatorname{Diff}(M) \times \operatorname{Diff}(M)$. Let $\operatorname{Diff}_{0}(M)$ be the connected component of the identity in $\operatorname{Diff}(M)$ with respect to this topology.

Theorem. For a Whitney manifold germ $M \subset \tilde{M}$ we actually have

$$
\operatorname{Diff}_{0}(M)=\left\{\left.\tilde{\varphi}\right|_{M}: \tilde{\varphi} \in \operatorname{Diff}_{0}(\tilde{M}), \tilde{\varphi}(M)=M\right\} .
$$

Consequently, the subgroup

$$
\operatorname{Diff}^{\sim}(M)=\left\{\left.\tilde{\varphi}\right|_{M}: \tilde{\varphi} \in \operatorname{Diff}(\tilde{M}), \tilde{\varphi}(M)=M\right\}
$$

is an open subgroup in $\operatorname{Diff}(M)$ and thus a normal subgroup, and the corresponding generating set $\mathcal{C}$ of smooth curves in $\operatorname{Diff}^{\sim}(M)$ is saturated.

Proof. Let $\varphi \in \operatorname{Diff}_{0}(M)$. Then there exists a smooth curve $\varphi: \mathbb{R} \rightarrow \operatorname{Diff}(M)$ with $\varphi(0)=\operatorname{Id}$ and $\varphi(1)=\varphi$ of the form $\varphi=\left.\tilde{c}\right|_{\mathbb{R} \times M}$ where $\tilde{c}: \mathbb{R} \times \tilde{M} \rightarrow \tilde{M}$ is a smooth mapping with $\left.\tilde{c}(t)\right|_{M,} \in \operatorname{Diff}(M)$ for each $t \in \mathbb{R}$. Then $X(t, x)=$ $\left(\partial_{t} \varphi(t)\right)\left(\varphi(t)^{-1}(x)\right)$ gives us a time-dependent vector field which is defined on $[0,1] \times U$ for some open neighborhood $U$ of $M$ in $\tilde{M}$, by the definition of $\operatorname{Diff}(M)$ in 6.3. Using a continuous extension operator on $\left.X\right|_{[0,1] \times M}$ and a smooth bump function gives us a smooth time-dependent vector field $\tilde{X}$ : $[0,1] \times \tilde{M} \rightarrow T \tilde{M}$ with support in a fixed open neighborhood, say, such that $\left.\tilde{X}\right|_{[0,1] \times M}=\left.X\right|_{[0,1] \times M}$. Solving the ODE $\partial_{t} \tilde{\varphi}(t . x)=\tilde{X}(t, \tilde{\varphi}(t, x))$ on $\tilde{M}$ gives us for $t=1$ a diffeomorphism $\tilde{\varphi} \in \operatorname{Diff}(\tilde{M})$ which extends $\varphi$.

Given any $\varphi \in \operatorname{Diff}^{\sim}(M)$, the coset $\varphi$. $\operatorname{Diff}_{0}(M) \subset \operatorname{Diff}(M)$ is the connected component of $\varphi$ in $\operatorname{Diff}(M)$. This shows that $\operatorname{Diff}^{\sim}(M)$ is open in $\operatorname{Diff}(M)$.

The construction in the proof above actually describes a smooth mapping

$$
\mathcal{E}:\left\{c \in C^{\infty}\left(\mathbb{R}, \operatorname{Diff}_{0}(M)\right): c(0)=\operatorname{Id}\right\} \rightarrow\left\{\tilde{\varphi} \in \operatorname{Diff}_{0}(\tilde{M}): \tilde{\varphi}(M)=M\right\}
$$

such that $\left.\mathcal{E}(c)\right|_{M}=c(1)$, since another smooth real parameter $s$ goes smoothly through solving the ODE.

6.6. Remark. In this paper I refrain from trying to give a general definition of a regular Frölicher group, which would be an abstract concept that catches the essential properties of $\operatorname{Diff}(M)$ for a Whitney manifold germ $M \subset \tilde{M}$. Let me just remark, that it probably would fit into the concept of manifolds based on smooth curves instead of charts as developed in [72]; those among them whose tangent spaces are Banach spaces turn out to be Banach manifolds. Some Lie theoretic tools are developed in the beginning of Section 8.5 below. 
6.7. Regular (right) half-Lie groups. A smooth manifold $G$ modeled on convenient vector spaces is called a (right) half Lie group, if it is a group such that multiplication $\mu: G \times G \rightarrow G$ and inversion $\nu: G \rightarrow G$ are continuous (note that here we have to take the induced $c^{\infty}$-topology on the product $G \times G$ if the model spaces are not Fréchet), but each right translation $\mu^{x}: G \rightarrow G$, $\mu^{x}(y)=y . x$ is smooth. The notion of a half-Lie group was coined in 60. See 64 for a study of half-Lie groups in general, concentrating on semidirect products with representation spaces.

Not every tangent vector in $T_{e} G$ can be extended to a left invariant vector field on the whole group, but they can be extended to right invariant vector fields, which are only continuous and not differentiable in general. The same holds for right invariant Riemannian metrics. The tangent space at the identity is not a Lie algebra in general; thus we refrain from calling it $\mathfrak{g}$. Have a look at the examples in 6.8 to get a feeling for this.

Let us discuss regularity on a (right) half-Lie group $G$ : For a smooth curve $g: \mathbb{R} \rightarrow G$ the velocity curve $g^{\prime}: \mathbb{R} \rightarrow T G$ is still smooth, and for fixed $t$ the right logarithmic derivative $X(t):=g^{\prime}(t) \cdot g(t)^{-1}=T\left(\mu^{g(t)^{-1}}\right) \cdot g^{\prime}(t)$ lies in $T_{e} G$, but $t \mapsto X(t)$ is only continuous $\mathbb{R} \rightarrow T_{e} G$. A (right) half-Lie group $G$ is called $C^{0}$-regular if for every $C^{0}$-curve $X: \mathbb{R} \rightarrow T_{e} G$ there exists a $C^{1}$-curve $\mathrm{Evol}^{X}=g: \mathbb{R} \rightarrow G$ with $g(0)=e$ and $g^{\prime}(t)=X(t) \cdot g(t)=T\left(\mu^{g(t)}\right) . X(t)$. We also require that $X \mapsto \mathrm{Evol}^{X}$ is smooth $C^{0}\left(\mathbb{R}, T_{e} G\right) \rightarrow C^{1}(\mathbb{R}, G)$.

6.8. Theorem (Diffeomorphism groups of finite degrees of differentiability). (1) For a compact smooth manifold $M$, possibly with corners, and for any $n \in \mathbb{N}_{\geq 1}$ the group $\operatorname{Diff}_{C^{n}}(M)$ of $C^{n}$-diffeomorphism of $M$ is a $C^{0}$-regular half-Lie group.

(2) For a compact smooth manifold $M$, possibly with corners, and for any $s \geq \operatorname{dim} M / p+1$, the group $\operatorname{Diff}_{W^{s, p}}(M)$ of Sobolev $W^{s, p}$-diffeomorphism of $M$ is a $C^{0}$-regular half-Lie group.

Note that the group of homeomorphisms of $M$ is not open in $C_{\text {nice }}^{0}(M, M)$; see the proof below for $C_{\text {nice }}^{\infty}$. Also note that $T_{\mathrm{Id}} \operatorname{Diff}_{C^{n}}(M)=\mathfrak{X}_{\partial, C^{n}}(M)$ is the space of $C^{n}$-vector fields which are tangent to the boundary. This is not a Lie algebra, since the Lie bracket of two $C^{n}$ fields is a $C^{n-1}$ field in general.

Proof. (1) Following [69, 10.16], we construct the smooth manifold structure by using the exponential mapping of a spray on $M$ which is tangential to the boundary; for existence see 3.7 and 5.9 Let $C_{\text {nice }}^{n}(M, M$,$) be the set$ of all $C^{n}$-mappings $f: M \rightarrow M$ with $f^{-1}\left(\partial^{q} M\right)=\partial^{q} M$ for each $q$. Then we use the (restriction of the) chart structure described in 5.3, using this exponential mappings, and using only charts centered at smooth mappings $f \in C_{\text {nice }}^{\infty}(M, M)$, as follows:

$$
\begin{gathered}
C_{\text {nice }}^{n}(M, N) \supset U_{f}=\left\{g:(f, g)(M) \subset V^{M \times M}\right\} \stackrel{u_{f}}{\longrightarrow} \tilde{U}_{f} \subset \\
\subset\left\{s \in C^{n}(M, T M): \pi_{M} \circ s=f, s\left(\partial^{q} M\right) \subset T\left(\partial^{q} M\right)\right\} \subset \Gamma_{C^{n}}\left(f^{*} T \tilde{M}\right), \\
u_{f}(g)=\left(\pi_{N}, \exp ^{\bar{g}}\right)^{-1} \circ(f, g), \quad u_{f}(g)(x)=\left(\exp _{f(x)}^{\bar{g}}\right)^{-1}(g(x)),
\end{gathered}
$$




$$
\left(u_{f}\right)^{-1}(s)=\exp _{f}^{\bar{g}} \circ s, \quad\left(u_{f}\right)^{-1}(s)(x)=\exp _{f(x)}^{\bar{g}}(s(x)) .
$$

By the symmetry of $V^{M \times M}$ (see 5.3 these charts cover $C_{\text {nice }}^{n}(M, M)$, and the chart changes are smooth since they map smooth curves (as described in 5.1.2) to smooth curves; compare to 5.7. The group $\operatorname{Diff}_{C^{n}}(M)$ is open in $C_{\text {nice }}^{n}(M, M)$, by the implicit function theorem and some easy arguments.

Continuity of composition and inversion are easy to check. Right translations are smooth since they map smooth curves.

$C^{1}$-regularity follows easily: Given $X \in C^{0}\left(\mathbb{R}, T_{\mathrm{Id}} \operatorname{Diff}_{C^{n}}(M)\right)$, view it as a time-dependent $C^{n}$-vector field on $M$ which is tangential to the boundary, a continuous curve in $\mathfrak{X}_{\partial}(M)$ and solve the corresponding ODE. The evolution operator Evol is smooth, since it maps smooth curves to smooth curves by standard ODE-arguments.

(2) This follows easily by adapting the proof of (1) above, using that $\operatorname{Diff}_{W^{s, p}} M \subset \operatorname{Diff}_{C^{1}}(M)$ by the Sobolev embedding lemma.

6.9. Groups of smooth diffeomorphisms on $\mathbb{R}^{n}$. If we consider the group of all orientation preserving diffeomorphisms $\operatorname{Diff}\left(\mathbb{R}^{n}\right)$ of $\mathbb{R}^{n}$, it is not an open subset of $C^{\infty}\left(\mathbb{R}^{n}, \mathbb{R}^{n}\right)$ with the compact $C^{\infty}$-topology. So it is not a smooth manifold in the usual sense, but we may consider it as a Lie group in the cartesian closed category of Frölicher spaces, see [55, Section 23], with the structure induced by the injection $f \mapsto\left(f, f^{-1}\right) \in C^{\infty}\left(\mathbb{R}^{n}, \mathbb{R}^{n}\right) \times C^{\infty}\left(\mathbb{R}^{n}, \mathbb{R}^{n}\right)$. Or one can use the setting of 'manifolds' based on smooth curves instead of charts, with lots of extra structure (tangent bundle, parallel transport, geodesic structure), described in [72]; this gives a category of smooth 'manifolds' where those which have Banach spaces as tangent fibes are exactly the usual smooth manifolds modeled on Banach spaces, which is cartesian closed: $C^{\infty}(M, N)$ and $\operatorname{Diff}(M)$ are always 'manifolds' for 'manifolds' $M$ and $N$, and the exponential law holds.

We shall now describe regular Lie groups in $\operatorname{Diff}\left(\mathbb{R}^{n}\right)$ which are given by diffeomorphisms of the form $f=\operatorname{Id}_{\mathbb{R}}+g$ where $g$ is in some specific convenient vector space of bounded functions in $C^{\infty}\left(\mathbb{R}^{n}, \mathbb{R}^{n}\right)$. Now we discuss these spaces on $\mathbb{R}^{n}$, we describe the smooth curves in them, and we describe the corresponding groups. These results are from [77] and from 60, 61] for the more exotic groups.

The group $\operatorname{Diff}_{\mathcal{B}}\left(\mathbb{R}^{n}\right)$. The space $\mathcal{B}\left(\mathbb{R}^{n}\right)$ (called $\mathcal{D}_{L^{\infty}}\left(\mathbb{R}^{n}\right)$ by [96]) consists of all smooth functions which have all derivatives (separately) bounded. It is a Fréchet space. By [105, the space $\mathcal{B}\left(\mathbb{R}^{n}\right)$ is linearly isomorphic to $\ell^{\infty} \hat{\otimes} \mathfrak{s}$ for any completed tensor-product between the projective one and the injective one, where $\mathfrak{s}$ is the nuclear Fréchet space of rapidly decreasing real sequences. Thus $\mathcal{B}\left(\mathbb{R}^{n}\right)$ is not reflexive, not nuclear, not smoothly paracompact.

The space $C^{\infty}\left(\mathbb{R}, \mathcal{B}\left(\mathbb{R}^{n}\right)\right)$ of smooth curves in $\mathcal{B}\left(\mathbb{R}^{n}\right)$ consists of all functions $c \in C^{\infty}\left(\mathbb{R}^{n+1}, \mathbb{R}\right)$ satisfying the following property:

- For all $k \in \mathbb{N}_{\geq 0}, \alpha \in \mathbb{N}_{\geq 0}^{n}$ and each $t \in \mathbb{R}$ the expression $\partial_{t}^{k} \partial_{x}^{\alpha} c(t, x)$ is uniformly bounded in $x \in \mathbb{R}^{n}$, locally in $t$. 
To see this use Theorem 2.6 for the set $\left\{\mathrm{ev}_{x}: x \in \mathbb{R}\right\}$ of point evaluations in $\mathcal{B}\left(\mathbb{R}^{n}\right)$. Here $\partial_{x}^{\alpha}=\frac{\partial^{|\alpha|}}{\partial x^{\alpha}}$ and $c^{k}(t)=\partial_{t}^{k} f(t, \quad)$.

$\operatorname{Diff}_{\mathcal{B}}^{+}\left(\mathbb{R}^{n}\right)=\left\{f=\operatorname{Id}+g: g \in \mathcal{B}\left(\mathbb{R}^{n}\right)^{n}, \operatorname{det}\left(\mathbb{I}_{n}+d g\right) \geq \varepsilon>0\right\}$ denotes the corresponding group, see below.

The group $\operatorname{Diff}_{W^{\infty, p}}\left(\mathbb{R}^{n}\right)$. For $1 \leq p<\infty$, the space

$$
W^{\infty, p}\left(\mathbb{R}^{n}\right)=\bigcap_{k \geq 1} L_{k}^{p}\left(\mathbb{R}^{n}\right)
$$

is the intersection of all $L^{p}$-Sobolev spaces, the space of all smooth functions such that each partial derivative is in $L^{p}$. It is a reflexive Fréchet space. It is called $\mathcal{D}_{L^{p}}\left(\mathbb{R}^{n}\right)$ in [96. By [105, the space $W^{\infty, p}\left(\mathbb{R}^{n}\right)$ is linearly isomorphic to $\ell^{p} \hat{\otimes} \mathfrak{s}$. Thus it is not nuclear, not Schwartz, not Montel, and smoothly paracompact only if $p$ is an even integer.

The space $C^{\infty}\left(\mathbb{R}, H^{\infty}\left(\mathbb{R}^{n}\right)\right)$ of smooth curves in $W^{\infty, p}\left(\mathbb{R}^{n}\right)$ consists of all functions $c \in C^{\infty}\left(\mathbb{R}^{n+1}, \mathbb{R}\right)$ satisfying the following property:

- For all $k \in \mathbb{N}_{\geq 0}, \alpha \in \mathbb{N}_{\geq 0}^{n}$ the expression $\left\|\partial_{t}^{k} \partial_{x}^{\alpha} f(t, \quad)\right\|_{L^{p}\left(\mathbb{R}^{n}\right)}$ is locally bounded near each $\bar{t} \in \mathbb{R}$.

The proof is literally the same as for $\mathcal{B}\left(\mathbb{R}^{n}\right)$, noting that the point evaluations are continuous on each Sobolev space $L_{k}^{p}$ with $k>\frac{n}{p}$.

$\operatorname{Diff}_{W \infty, p}^{+}\left(\mathbb{R}^{n}\right)=\left\{f=\mathrm{Id}+g: g \in W^{\infty, p}\left(\mathbb{R}^{n}\right)^{n}, \operatorname{det}\left(\mathbb{I}_{n}+d g\right)>0\right\}$ denotes the corresponding group.

The group Diff $\mathcal{S}\left(\mathbb{R}^{n}\right)$. The algebra $\mathcal{S}\left(R^{n}\right)$ of rapidly decreasing functions is a reflexive nuclear Fréchet space.

The space $C^{\infty}\left(\mathbb{R}, \mathcal{S}\left(\mathbb{R}^{n}\right)\right)$ of smooth curves in $\mathcal{S}\left(\mathbb{R}^{n}\right)$ consists of all functions $c \in C^{\infty}\left(\mathbb{R}^{n+1}, \mathbb{R}\right)$ satisfying the following property:

- For all $k, m \in \mathbb{N}_{\geq 0}$ and $\alpha \in \mathbb{N}_{\geq 0}^{n}$, the expression $\left(1+|x|^{2}\right)^{m} \partial_{t}^{k} \partial_{x}^{\alpha} c(t, x)$ is uniformly bounded in $x \in \mathbb{R}^{n}$, locally uniformly bounded in $t \in \mathbb{R}$.

$\operatorname{Diff}_{\mathcal{S}}^{+}\left(\mathbb{R}^{n}\right)=\left\{f=\mathrm{Id}+g: g \in \mathcal{S}\left(\mathbb{R}^{n}\right)^{n}, \operatorname{det}\left(\mathbb{I}_{n}+d g\right)>0\right\}$ is the corresponding group.

The group Diff $c\left(\mathbb{R}^{n}\right)$. The algebra $C_{c}^{\infty}\left(\mathbb{R}^{n}\right)$ of all smooth functions with compact support is a nuclear (LF)-space.

The space $C^{\infty}\left(\mathbb{R}, C_{c}^{\infty}\left(\mathbb{R}^{n}\right)\right)$ of smooth curves in $C_{c}^{\infty}\left(\mathbb{R}^{n}\right)$ consists of all functions $f \in C^{\infty}\left(\mathbb{R}^{n+1}, \mathbb{R}\right)$ satisfying the following property:

- For each compact interval $[a, b]$ in $\mathbb{R}$ there exists a compact subset $K \subset \mathbb{R}^{n}$ such that $f(t, x)=0$ for $(t, x) \in[a, b] \times\left(\mathbb{R}^{n} \backslash K\right)$.

$\operatorname{Diff}_{c}\left(\mathbb{R}^{n}\right)=\left\{f=\mathrm{Id}+g: g \in C_{c}^{\infty}\left(\mathbb{R}^{n}\right)^{n}, \operatorname{det}\left(\mathbb{I}_{n}+d g\right)>0\right\}$ is the corresponding group. The case $\operatorname{Diff}_{c}\left(\mathbb{R}^{n}\right)$ is well-known since 1980 .

Ideal properties of function spaces. The function spaces discussed are boundedly mapped into each other as follows:

$$
C_{c}^{\infty}\left(\mathbb{R}^{n}\right) \longrightarrow \mathcal{S}\left(\mathbb{R}^{n}\right) \longrightarrow W^{\infty, p}\left(\mathbb{R}^{n}\right) \stackrel{p \leq q}{\longrightarrow} W^{\infty, q}\left(\mathbb{R}^{n}\right) \longrightarrow \mathcal{B}\left(\mathbb{R}^{n}\right)
$$


and each space is a bounded locally convex algebra and a bounded $\mathcal{B}\left(\mathbb{R}^{n}\right)$ module. Thus each space is an ideal in each larger space.

6.10. Theorem (77] and 60). The sets of diffeomorphisms

$$
\operatorname{Diff}_{c}\left(\mathbb{R}^{n}\right), \quad \operatorname{Diff}_{\mathcal{S}}\left(\mathbb{R}^{n}\right), \quad \operatorname{Diff}_{H^{\infty}}\left(\mathbb{R}^{n}\right), \text { and } \operatorname{Diff}_{\mathcal{B}}\left(\mathbb{R}^{n}\right)
$$

are all smooth regular Lie groups. We have the following smooth injective group homomorphisms

$$
\operatorname{Diff}_{c}\left(\mathbb{R}^{n}\right) \longrightarrow \operatorname{Diff}_{\mathcal{S}}\left(\mathbb{R}^{n}\right) \longrightarrow \operatorname{Diff}_{W \infty, p}\left(\mathbb{R}^{n}\right) \longrightarrow \operatorname{Diff}_{\mathcal{B}}\left(\mathbb{R}^{n}\right) .
$$

Each group is a normal subgroup in any other in which it is contained, in particular in $\operatorname{Diff}_{\mathcal{B}}\left(\mathbb{R}^{n}\right)$.

The proof of this theorem relies on repeated use of the Faà di Bruno formula for higher derivatives of composed functions. This offers difficulties on non-compact manifolds, where one would need a non-commutative Faà di Bruno formula for iterated covariant derivatives. In the paper 60] many more similar groups are discussed, modeled on spaces of Denjoy-Carleman ultradifferentiable functions. It is also shown that for $p>1$ the group Diff $_{W^{\infty, p} \cap L^{1}}\left(\mathbb{R}^{n}\right)$ is only a topological group with smooth right translations - a property which is similar to the one of finite order Sobolev groups $\operatorname{Diff}_{W^{k, p}}\left(\mathbb{R}^{n}\right)$. Some of these groups were used extensively in 80.

6.11. Corollary. Diff $\mathcal{B}_{\mathcal{B}}\left(\mathbb{R}^{n}\right)$ acts on $\Gamma_{c}, \Gamma_{\mathcal{S}}$ and $\Gamma_{H^{\infty}}$ of any tensor bundle over $\mathbb{R}^{n}$ by pullback. The infinitesimal action of the Lie algebra $\mathfrak{X}_{\mathcal{B}}\left(\mathbb{R}^{n}\right)$ on these spaces by the Lie derivative maps each of these spaces into itself. A fortiori, Diff $H^{\infty}\left(\mathbb{R}^{n}\right)$ acts on $\Gamma_{\mathcal{S}}$ of any tensor bundle by pullback.

6.12. Trouvé groups. For the following see [103, [108, 85]. Trouvé groups are useful for introducing topological metrics on certain groups of diffeomorphism on $\mathbb{R}^{d}$ starting from a suitable reproducing kernel Hilbert space of vector fields without using any Lie algebra structure; see 8.12 below.

Consider a time-dependent vector field $X:[0,1] \times \mathbb{R}^{d} \rightarrow \mathbb{R}$ of sufficient regularity (e.g, continuous in $t \in[0,1]$ and Lipschitz continuous in $x \in \mathbb{R}^{d}$ with $t$-integrable global Lipschitz constant) so that

$$
x(t)=x_{0}+\int_{0}^{t} X(s, x(s)) d s
$$

is uniquely solvable for all $t \in[0,1]$ and $x_{0} \in \mathbb{R}^{d}$. Then we consider the evolution evol $^{X}\left(x_{0}\right)=x(1)$. For $X \in L^{1}\left([0,1], C_{b}^{1}\left(\mathbb{R}^{d}, \mathbb{R}\right)^{d}\right.$ ) (where $f \in C_{b}^{k}$ if all iterated partial derivatives of order between 0 and $k$ are continuous and globally bounded) we have evol ${ }^{X} \in \mathrm{Id}+C_{b}^{1}\left(\mathbb{R}^{d}, \mathbb{R}^{d}\right)$ and is a diffeomorphism with $(\text { evol })^{-1} \in \mathrm{Id}+C_{b}^{1}\left(\mathbb{R}^{d}, \mathbb{R}^{d}\right)$. Given a convenient locally convex vector space $\mathcal{A}\left(\mathbb{R}^{d}, \mathbb{R}^{d}\right)$ of mappings $\mathbb{R}^{d} \rightarrow \mathbb{R}^{d}$ which continuously embeds into $C_{b}^{1}\left(\mathbb{R}^{d}, \mathbb{R}^{d}\right)$ and a suitable family of mappings $[0,1] \rightarrow \mathcal{A}\left(\mathbb{R}^{d}, \mathbb{R}^{d}\right)$, the associated Trouvé group is given by

$$
\mathcal{G}_{\mathcal{A}}:=\left\{\operatorname{evol}^{X}: X \in \mathcal{F}_{\mathcal{A}}\right\}
$$


where $\mathcal{F}_{\mathcal{A}}=\mathcal{F}\left([0,1], \mathcal{A}\left(\mathbb{R}^{d}, \mathbb{R}^{d}\right)\right)$ is a suitable vector space of time-dependent vector fields. It seems that for a wide class of spaces $\mathcal{A}$ the Trouvé group $\mathcal{G}_{\mathcal{A}}$ is independent of the choice of $\mathcal{F}_{\mathcal{A}}$ if the latter contains the piecewise smooth curves and is contained in the curves which are integrable by seminorms; a precise statement is still lacking, but see [85, 84, 82, and citations therein. The space $\mathcal{A}$ is called $\mathcal{F}_{\mathcal{A}^{-}} O D E$-closed if evol ${ }^{X} \in \mathrm{Id}+\mathcal{A}\left(\mathbb{R}^{d}, \mathbb{R}^{d}\right)$ for each $X \in$ $\mathcal{F}_{\mathcal{A}}$. For ODE-closed $\mathcal{A}$ the Trouvé group $\mathcal{G}_{\mathcal{A}}$ is contained in $\operatorname{Id}+\mathcal{A}\left(\mathbb{R}^{d}, \mathbb{R}^{d}\right)$.

For some spaces $\mathcal{A}$ it has been proved that $\mathcal{F}_{\mathcal{A}}$ is equal to the connected component of the identity of

$$
\left\{\operatorname{Id}+f: f \in \mathcal{A}\left(\mathbb{R}^{d}, \mathbb{R}^{d}\right), \inf _{x \in \mathbb{R}^{d}} \operatorname{det} d f(x)>-1\right\},
$$

namely:

- For Sobolev spaces $W^{k, 2}$ with $k>d / 2$ by [22]; $\mathcal{G}_{\mathcal{A}}$ is a half Lie group.

- For Hölder spaces by [84.

- For Besov spaces by 83 .

- For $\mathcal{B}, W^{\infty, p}$, Schwartz functions $\mathcal{S}, C_{c}^{\infty}$, and many classes of DenjoyCarleman functions, where $\mathcal{G}_{\mathcal{A}}$ is always a regular Lie group; see [85.

\section{SpaCes of EMbedDings or IMmersions, AND SHAPE SPACES}

This is the main section in this chapter.

7.1. The principal bundle of embeddings. For finite dimensional manifolds $M, N$ with $M$ compact, $\operatorname{Emb}(M, N)$, the space of embeddings of $M$ into $N$, is open in $C^{\infty}(M, N)$, so it is a smooth manifold. Diff $(M)$ acts freely and smoothly from the right on $\operatorname{Emb}(M, N)$.

Theorem. $\operatorname{Emb}(M, N) \rightarrow \operatorname{Emb}(M, N) / \operatorname{Diff}(M)=B(M, N)$ is a smooth principal fiber bundle with structure group $\operatorname{Diff}(M)$. Its base is a smooth manifold.

This result was proved in [70 for $M$ an open manifold without boundary; see also [69. Note that $B(M, N)$ is the smooth manifold of all submanifolds of $N$ which are of diffeomorphism type $M$. Therefore it is also called the nonlinear Grassmannian in [45], where this theorem is extended to the case when $M$ has boundary. From another point of view, $B(M, N)$ is called the differentiable Chow variety in [68. It is an example of a shape space.

Proof. We use an auxiliary Riemannian metric $\bar{g}$ on $N$. Given an embedding $f \in \operatorname{Emb}(M, N)$, we view $f(M)$ as a submanifold of $N$ and we split the tangent bundle of $N$ along $f(M)$ as $\left.T N\right|_{f(M)}=\operatorname{Nor}(f(M)) \oplus T f(M)$. The exponential mapping describes a tubular neighborhood of $f(M)$ via

$$
\operatorname{Nor}(f(M)) \stackrel{\exp ^{\bar{g}}}{\cong} W_{f(M)} \stackrel{p_{f(M)}}{\longrightarrow} f(M) .
$$

If $g: M \rightarrow N$ is $C^{1}$-near to $f$, then $\varphi(g):=f^{-1} \circ p_{f(M)} \circ g \in \operatorname{Diff}(M)$ and we may consider $g \circ \varphi(g)^{-1} \in \Gamma\left(f^{*} W_{f(M)}\right) \subset \Gamma\left(f^{*} \operatorname{Nor}(f(M))\right)$. This is the required local splitting. 
7.2. The space of immersions and the space of embeddings of a compact Whitney manifold germ. Let $\tilde{M} \supset M$ be a compact Whitney manifold germ, and let $N$ be a smooth manifold with $\operatorname{dim}(M) \leq \operatorname{dim}(N)$. We define the space of immersions as

$$
\operatorname{Imm}(M, N)=\left\{f=\left.\tilde{f}\right|_{M}, f \in C^{\infty}(\tilde{M}, N), T_{x} \tilde{f} \text { is injective for } x \in M\right\}
$$

which is open in the smooth manifold $C^{\infty}(M, N)$ and is thus itself a smooth manifold. Note that any extension of an immersion $f \in \operatorname{Imm}(M, N)$ to $\tilde{f} \in C^{\infty}(\tilde{M}, N)$ is still an immersion on an open neighborhood of $M$ in $\tilde{M}$.

Likewise we let

$$
\begin{gathered}
\operatorname{Emb}(M, N)=\left\{\left.f\right|_{M}, f \in C^{\infty}(\tilde{M}, N), T_{x} f \text { is injective for } x \in M,\right. \\
f: M \rightarrow N \text { is a topological embedding }\}
\end{gathered}
$$

Since $M$ is compact, any extension of an embedding $f \in \operatorname{Emb}(M, N)$ to $\tilde{f} \in C^{\infty}(\tilde{M}, N)$ is an embedding on some open neighborhood of $M$ in $\tilde{M}$; see [69, 5.3] for a proof a related result.

Theorem. For a compact Whitney germ $M$ and a smooth manifold $N$ with $\operatorname{dim}(M)<\operatorname{dim}(N)$ the projection

$$
\pi: \operatorname{Emb}(M, N) \rightarrow \operatorname{Emb}(M, N) / \operatorname{Diff}(M)=B(M, N)
$$

is a smooth principal fiber bundle of Frölicher spaces with structure group the Frölicher group $\operatorname{Diff}(M)$ from 6.4. Its base is the quotient Frölicher space.

Proof. Since I do not know that $\operatorname{Diff}(M)$ is a smooth manifold, we treat all spaces here as Frölicher spaces. By definition, the right action of $\operatorname{Diff}(M)$ on $\operatorname{Emb}(M, N)$ is free, and smooth between the Frölicher spaces. The quotient $B(M, N)$ carries the quotient Frölicher structure with generating set of curves $\left\{\pi \circ c: c \in C^{\infty}(\mathbb{R}, \operatorname{Emb}(M, N))\right\}$, i.e., those which lift to a smooth curve.

7.3. The orbifold bundle of immersions. Let $M$ be a (not necessarily compact) manifold without boundary. Let $N$ be an open manifold with $\operatorname{dim}(M) \leq \operatorname{dim}(N)$. Then $\operatorname{Imm}(M, N)$, the space of immersions $M \rightarrow N$, is open in $C^{\infty}(M, N)$, and is thus a smooth manifold. The regular Lie group (or Frölicher group if $M$ is a Whitney manifold germ) Diff( $M$ ) acts smoothly from the right, but no longer freely.

An immersion $i: M \rightarrow N$ is called free if $\operatorname{Diff}(M)$ acts freely on it: $i \circ f=i$ for $f \in \operatorname{Diff}(M)$ implies $f=\operatorname{Id}_{M}$.

The space $B_{i}(M, N)=\operatorname{Imm}(M, N) / \operatorname{Diff}(M)$ is an example of a shape space. It appeared in the form of $B_{i}\left(S^{1}, \mathbb{R}^{2}\right)$, the shape space of plane immersed curves, in 75 and 76]. The following theorem is essentially due to 23 ; since this paper contains some annoying misprints and is difficult to understand, we give here an extended version with a more detailed proof. The reader may skip this proof and jump directly to 7.2 below.

Theorem ([23]). Let $M$ be a finite dimensional smooth manifold. Let $N$ be smooth finite dimensional manifolds with $\operatorname{dim}(M) \leq \operatorname{dim}(N)$. Then the following holds: 
(1) The diffeomorphism group $\operatorname{Diff}(M)$ acts smoothly from the right on the manifold $\operatorname{Imm}_{\text {prop }}(M, N)$ of all smooth proper immersions $M \rightarrow$ $N$, which is an open subset of $C^{\infty}(M, N)$.

(2) The space of orbits $\operatorname{Imm}_{\text {prop }}(M, N) / \operatorname{Diff}(M)$ is Hausdorff in the quotient topology.

(3) The set $\operatorname{Imm}_{\text {free, } \operatorname{prop}}(M, N)$ of all proper free immersions is open in $C^{\infty}(M, N)$ and is the total space of a smooth principal fiber bundle $\operatorname{Imm}_{\text {free,prop }}(M, N) \rightarrow \operatorname{Imm}_{\text {free,prop }}(M, N) / \operatorname{Diff}(M)$.

(4) Let $i \in \operatorname{Imm}(M, N)$ be an immersion which is not free. So we have a nontrivial isotropy subgroup $\operatorname{Diff}(M)_{i} \subset \operatorname{Diff}(M)$ consisting of all $f \in \operatorname{Diff}(M)$ with $i \circ f=i$. Then the isotropy group $\operatorname{Diff}(M)_{i}$ acts properly discontinuously on $M$. Thus the projection $q_{1}: M \rightarrow M_{1}:=$ $M / \operatorname{Diff}(M)_{i}$ is a covering mapping onto a smooth manifold $M_{1}$. There exists an immersion $i_{1}: M_{1} \rightarrow N$ with $i=i_{1} \circ q_{1}$. In particular, $\operatorname{Diff}(M)_{i}$ is countable, and is finite if $M$ is compact. There exists a further covering $q_{2}: M \rightarrow M_{1} \rightarrow M_{2}$ and a free immersion $i_{2}: M_{2} \rightarrow N$ with $i=i_{2} \circ q_{2}$.

(5) Let $M$ have the property that for any covering $M \rightarrow M_{1}$ of smooth manifolds, any diffeomorphism $M_{1} \rightarrow M_{1}$ admits a lift $M \rightarrow M$; e.g., $M$ simply connected, or $M=S^{1}$. Let $i \in \operatorname{Imm}(M, N)$ be an immersion which is not free, i.e., has non-trivial isotropy group $\operatorname{Diff}(M)_{i}$, and let $q_{1}: M \rightarrow M_{1}:=M / \operatorname{Diff}(M)_{i}$ be the corresponding covering map. Then in the following commutative diagram the bottom mapping

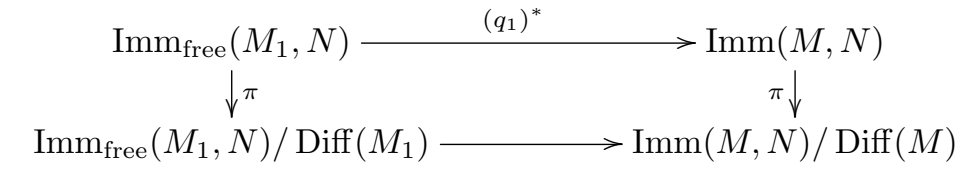

is the inclusion of a (possibly non-Hausdorff) manifold, the stratum of $\pi(i)$ in the stratification of the orbit space. This stratum consists of the orbits of all immersions which have $\operatorname{Diff}(M)_{i}$ as isotropy group. See (23) and (24) below for a more complete description of the orbit structure near $i$.

(6) [100] We have a right action of $\operatorname{Diff}(M)$ on $\operatorname{Imm}(M, N) \times M$ which is given by $(i, x) . f=\left(i \circ f, f^{-1}(x)\right)$. This action is free.

$(\operatorname{Imm}(M, N) \times M, \pi,(\operatorname{Imm}(M, N) \times M) / \operatorname{Diff}(M), \operatorname{Diff}(M))$

is a smooth principal fiber bundle with structure group $\operatorname{Diff}(M)$ and a smooth base manifold $S(M, N):=(\operatorname{Imm}(M, N) \times M) / \operatorname{Diff}(M)$ which might possibly be non-Hausdorff. If we restrict to the open subset $\operatorname{Imm}_{\text {prop }}(M, N) \times M$ of proper immersions times $M$ then the base space is Hausdorff.

Proof. Without loss, let $M$ be connected. Fix an immersion $i: M \rightarrow N$. We will now describe some data for $i$ which we will use throughout the proof. 
If we need these data for several immersions, we will distinguish them by appropriate superscripts.

(7) Setup. There exist sets $W_{\alpha} \subset \bar{W}_{\alpha} \subset U_{\alpha} \subset \bar{U}_{\alpha} \subset V_{\alpha} \subset M$ such that $\left(W_{\alpha}\right)$ is an open cover of $M, \bar{W}_{\alpha}$ is compact, and $V_{\alpha}$ is an open locally finite cover of $M$, each $W_{\alpha}, U_{\alpha}$, and $V_{\alpha}$ is connected, and such that $i \mid V_{\alpha}: V_{\alpha} \rightarrow N$ is an embedding for each $\alpha$.

Let $g$ be a fixed Riemannian metric on $N$ and let $\exp ^{N}$ be the induced geodesic exponential mapping. Then let $p: \mathcal{N}(i) \rightarrow M$ be the normal bundle of $i$, defined in the following way: For $x \in M$ let $\mathcal{N}(i)_{x}:=\left(T_{x} i\left(T_{x} M\right)\right)^{\perp} \subset$ $T_{i(x)} N$ be the $g$-orthogonal complement in $T_{i(x)} N$. Then

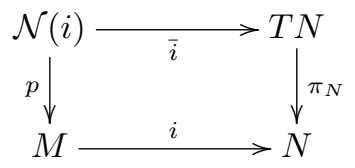

is a vector bundle homomorphism over $i$, which is fiberwise injective.

Now let $U^{i}=U$ be an open neighborhood of the zero section of $\mathcal{N}(i)$ which is so small that $\left(\exp ^{N} \circ \bar{i}\right)\left|\left(\left.U\right|_{V_{\alpha}}\right): U\right|_{V_{\alpha}} \rightarrow N$ is a diffeomorphism onto its image which describes a tubular neighborhood of the submanifold $i\left(V_{\alpha}\right)$ for each $\alpha$. Let

$$
\tau=\tau^{i}:=\left.\left(\exp ^{N} \circ \bar{i}\right)\right|_{U}: \mathcal{N}(i) \supset U \rightarrow N .
$$

It will serve us as a substitute for a tubular neighborhood of $i(M)$.

For any $f \in \operatorname{Diff}(M)_{i}=\{f \in \operatorname{Diff}(M): i \circ f=i\}$ we have an induced vector bundle homomorphism $\bar{f}$ over $f$ :

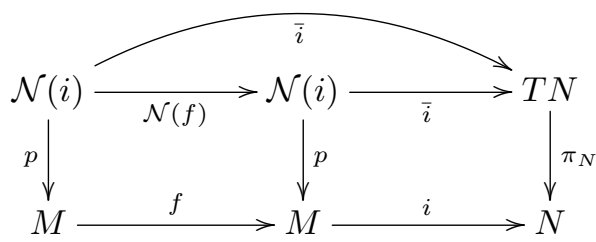

(8) Claim. Let $i \in \operatorname{Imm}(M, N)$ and let $f \in \operatorname{Diff}(M)$ have a fixed point $x_{0} \in M$ and satisfy $i \circ f=i$. Then $f=I d_{M}$.

Namely, we consider the sets $\left(U_{\alpha}\right)$ for the immersion $i$ of (7). Let us investigate $f\left(U_{\alpha}\right) \cap U_{\alpha}$. If there is an $x \in U_{\alpha}$ with $y=f(x) \in U_{\alpha}$, we have $\left(\left.i\right|_{U_{\alpha}}\right)(x)=\left((i \circ f) \mid U_{\alpha}\right)(x)=\left(\left.i\right|_{U_{\alpha}}\right)(f(x))=\left(\left.i\right|_{U_{\alpha}}\right)(y)$. Since $\left.i\right|_{U_{\alpha}}$ is injective we have $x=y$, and

$$
f\left(U_{\alpha}\right) \cap U_{\alpha}=\left\{x \in U_{\alpha}: f(x)=x\right\} .
$$

Thus $f\left(U_{\alpha}\right) \cap U_{\alpha}$ is closed in $U_{\alpha}$. Since it is also open and since $U_{\alpha}$ is connected, we have $f\left(U_{\alpha}\right) \cap U_{\alpha}=\emptyset$ or $=U_{\alpha}$.

Now we consider the set $\{x \in M: f(x)=x\}$. We have just shown that it is open in $M$. Since it is also closed and contains the fixed point $x_{0}$, it coincides with $M$. Claim (7) follows. 
(9) Claim. If for an immersion $i \in \operatorname{Imm}(M, N)$ there is a point in $i(M)$ with only one preimage, then $i$ is a free immersion.

Let $x_{0} \in M$ be such that $i\left(x_{0}\right)$ has only one preimage. If $i \circ f=i$ for $f \in \operatorname{Diff}(M)$ then $f\left(x_{0}\right)=x_{0}$ and $f=I d_{M}$ by claim (8).

Note that there are free immersions without a point in $i(M)$ with only one preimage: Consider a figure eight which consists of two touching circles. Now we may map the circle to the figure eight by going first $n$ times around the upper circle, then $m$ around the lower one with $n, m \geq 2$.

(10) Claim. Let $i$ be a free immersion $M \rightarrow N$. Then there is an open neighborhood $\mathcal{W}(i)$ in $\operatorname{Imm}(M, N)$ which is saturated for the $\operatorname{Diff}(M)$-action and which splits smoothly as

$$
\mathcal{W}(i)=\mathcal{Q}(i) \times \operatorname{Diff}(M) .
$$

Here $\mathcal{Q}(i)$ is a smooth splitting submanifold of $\operatorname{Imm}(M, N)$, diffeomorphic to an open neighborhood of the zero section in $\Gamma_{c}(M \leftarrow \mathcal{N}(i))$. In particular the space $\operatorname{Imm}_{\text {free }}(M, N)$ is open in $C^{\infty}(M, N)$.

Let $\pi: \operatorname{Imm}(M, N) \rightarrow \operatorname{Imm}(M, N) / \operatorname{Diff}(M)=B_{i}(M, N)$ be the projection onto the orbit space, which is equipped with the quotient topology. Then the mapping $\left.\pi\right|_{\mathcal{Q}(i)}: \mathcal{Q}(i) \rightarrow \pi(\mathcal{Q}(i))$ is bijective onto an open subset of the quotient. If $i$ runs through $\operatorname{Imm}_{\text {free,prop }}(M, N)$ of all free and proper immersions these mappings define a smooth atlas for the quotient space, so that

$$
\left(\operatorname{Imm}_{\text {free,prop }}(M, N), \pi, \operatorname{Imm}_{\text {free,prop }}(M, N) / \operatorname{Diff}(M), \operatorname{Diff}(M)\right)
$$

is a smooth principal fiber bundle with structure group $\operatorname{Diff}(M)$.

The restriction to proper immersions is necessary because we are only able to show that $\operatorname{Imm}_{\text {prop }}(M, N) / \operatorname{Diff}(M)$ is Hausdorff in (11) below.

For the proof of claim (10), we consider the setup (7) for the free immersion $i$. Let

$$
\tilde{\mathcal{U}}(i):=\left\{j \in \operatorname{Imm}(M, N): j\left(\bar{W}_{\alpha}^{i}\right) \subseteq \tau^{i}\left(\left.U^{i}\right|_{U_{\alpha}^{i}}\right) \text { for all } \alpha, j \sim i\right\},
$$

where $j \sim i$ means that $j=i$ off some compact set in $M$. Then by 5.3 (for open $M)$ the set $\tilde{\mathcal{U}}(i)$ is an open neighborhood of $i$ in $\operatorname{Imm}(M, N)$. For each $j \in \tilde{\mathcal{U}}(i)$ we define

$$
\begin{aligned}
& \varphi_{i}(j): M \rightarrow U^{i} \subseteq \mathcal{N}(i), \\
& \varphi_{i}(j)(x):=\left(\left.\tau^{i}\right|_{\left(\left.U^{i}\right|_{U_{\alpha}^{i}}\right)}\right)^{-1}(j(x)) \text { if } x \in W_{\alpha}^{i} .
\end{aligned}
$$

Note that $\varphi_{i}(j)$ is defined piecewise on $M$, but the pieces coincide when they overlap. Therefore a smooth curve through $j$ is mapped to a smooth curve and so $\varphi_{i}: \tilde{\mathcal{U}}(i) \rightarrow C^{\infty}(M, \mathcal{N}(i))$ is a smooth mapping which is bijective onto the open set

$$
\tilde{\mathcal{V}}(i):=\left\{h \in C^{\infty}(M, \mathcal{N}(i)):\left.h\left(\bar{W}_{\alpha}^{i}\right) \subseteq U^{i}\right|_{U_{\alpha}^{i}} \text { for all } \alpha, h \sim 0\right\}
$$


in $C^{\infty}(M, \mathcal{N}(i))$. Its inverse is given by the smooth mapping $\tau_{*}^{i}: h \mapsto \tau^{i} \circ h$. Now we consider the open subsets

$$
\begin{aligned}
& \mathcal{V}(i):=\left\{h \in \tilde{\mathcal{V}}(i): p \circ h \in \operatorname{Diff}_{c}(M)\right\} \subset \tilde{\mathcal{V}}(i) \\
& \mathcal{U}(i):=\tau_{*}^{i}(\mathcal{V}(i)) \subset \tilde{\mathcal{U}}(i)
\end{aligned}
$$

and the diffeomorphism $\varphi_{i}: \mathcal{U}(i) \rightarrow \mathcal{V}(i)$. For $h \in \mathcal{V}(i)$ we have $\tau_{*}^{i}(h \circ f)=$ $\tau_{*}^{i}(h) \circ f$ for those $f \in \operatorname{Diff}(M)$ which are near enough to the identity so that $h \circ f \in \mathcal{V}(i)$. And if $\tau^{i} \circ h \circ f=\tau^{i} \circ h$ then $h \circ f=h$ by the construction of $\mathcal{N}(i)$ in (7), and then $f=\operatorname{Id}_{\mathrm{M}}$ since $i$ is a free immersion; see the second diagram in (7).

We consider now the open set

$$
\{h \circ f: h \in \mathcal{V}(i), f \in \operatorname{Diff}(M)\} \subseteq C^{\infty}\left(M, U^{i}\right) .
$$

Consider the smooth mapping from it into $\Gamma_{c}\left(M \leftarrow U^{i}\right) \times \operatorname{Diff}(M)$ given by $h \mapsto\left(h \circ(p \circ h)^{-1}, p \circ h\right)$, where $\Gamma_{c}\left(M \leftarrow U^{i}\right)$ is the space of sections with compact support of $U^{i} \rightarrow M$. So if we let $\mathcal{Q}(i):=\tau_{*}^{i}\left(\Gamma_{c}\left(M \leftarrow U^{i}\right) \cap \mathcal{V}(i)\right) \subset$ $\operatorname{Imm}(M, N)$ we have

$\mathcal{W}(i):=\mathcal{U}(i) \circ \operatorname{Diff}_{c}(M) \cong \mathcal{Q}(i) \times \operatorname{Diff}(M) \cong\left(\Gamma_{c}\left(M \leftarrow U^{i}\right) \cap \mathcal{V}(i)\right) \times \operatorname{Diff}(M)$,

since the action of $\operatorname{Diff}(M)$ on $i$ is free and by the argument above. Consequently $\operatorname{Diff}(M)$ acts freely on each immersion in $\mathcal{W}(i)$, so $\operatorname{Imm}_{\text {free }}(M, N)$ is open in $C^{\infty}(M, N)$. Furthermore

$$
\left.\pi\right|_{\mathcal{Q}(i)}: \mathcal{Q}(i) \rightarrow \operatorname{Imm}_{\text {free }}(M, N) / \operatorname{Diff}(M)
$$

is bijective onto an open set in the quotient.

We consider

$$
\varphi_{i} \circ\left(\left.\pi\right|_{\mathcal{Q}(i)}\right)^{-1}: \pi(\mathcal{Q}(i)) \rightarrow \Gamma_{c}\left(M \leftarrow U^{i}\right) \subset C_{c}^{\infty}(N, \mathcal{N}(i))
$$

as a chart for the quotient space.

In order to investigate the chart change let $j \in \operatorname{Imm}_{\text {free }}(M, N)$ be such that $\pi(\mathcal{Q}(i)) \cap \pi(\mathcal{Q}(j)) \neq \emptyset$. Then there is an immersion $h \in \mathcal{W}(i) \cap \mathcal{Q}(j)$, so there exists a unique $f_{0} \in \operatorname{Diff}(M)$ (given by $f_{0}=p \circ \varphi_{i}(h)$ ) such that $h \circ f_{0}^{-1} \in \mathcal{Q}(i)$. If we consider $j \circ f_{0}^{-1}$ instead of $j$ and call it again $j$, we have $\mathcal{Q}(i) \cap \mathcal{Q}(j) \neq \emptyset$ and consequently $\mathcal{U}(i) \cap \mathcal{U}(j) \neq \emptyset$. Then the chart change is given as follows:

$$
\begin{gathered}
\varphi_{i} \circ\left(\left.\pi\right|_{\mathcal{Q}(i)}\right)^{-1} \circ \pi \circ\left(\tau^{j}\right)_{*}: \Gamma_{c}\left(M \leftarrow U^{j}\right) \rightarrow \Gamma_{c}\left(M \leftarrow U^{i}\right) \\
s \mapsto \tau^{j} \circ s \mapsto \varphi_{i}\left(\tau^{j} \circ s\right) \circ\left(p^{i} \circ \varphi_{i}\left(\tau^{j} \circ s\right)\right)^{-1} .
\end{gathered}
$$

This is of the form $s \mapsto \beta \circ s$ for a locally defined diffeomorphism $\beta: \mathcal{N}(j) \rightarrow$ $\mathcal{N}(i)$ which is not fiber respecting, followed by $h \mapsto h \circ\left(p^{i} \circ h\right)^{-1}$. Both composants are smooth by the general properties of manifolds of mappings. So the chart change is smooth.

We have to show that the quotient space $\operatorname{Imm}_{\text {prop,free }}(M, N) / \operatorname{Diff}(M)$ is Hausdorff. 
(11) Claim. The orbit space $\operatorname{Imm}_{\text {prop }}(M, N) / \operatorname{Diff}(M)$ of the space of all proper immersions under the action of the diffeomorphism group is Hausdorff in the quotient topology.

This follows from (18) below. I am convinced that the whole orbit space $\operatorname{Imm}(M, N) / \operatorname{Diff}(M)$ is Hausdorff, but I was unable to prove this.

(12) Claim. Let $i$ and $j \in \operatorname{Imm}_{\text {prop }}(M, N)$ with $i(M) \neq j(M)$ in $N$. Then their projections $\pi(i)$ and $\pi(j)$ are different and can be separated by open subsets in $\operatorname{Imm}_{\text {prop }}(M, N) / \operatorname{Diff}(M)$.

We suppose that $i(M) \nsubseteq \overline{j(M)}=j(M)$ (since proper immersions have closed images). Let $y_{0} \in i(M) \backslash \overline{j(M)}$, then we choose open neighborhoods $V$ of $y_{0}$ in $N$ and $W$ of $j(M)$ in $N$ such that $V \cap W=\emptyset$. We consider the sets

$$
\begin{aligned}
\mathcal{V} & :=\left\{k \in \operatorname{Imm}_{\text {prop }}(M, N): k(M) \cap V \neq \emptyset\right\} \quad \text { and } \\
\mathcal{W} & :=\left\{k \in \operatorname{Imm}_{\text {prop }}(M, N): k(M) \subseteq W\right\} .
\end{aligned}
$$

Then $\mathcal{V}$ and $\mathcal{W}$ are $\operatorname{Diff}(M)$-saturated disjoint open neighborhoods of $i$ and $j$, respectively, so $\pi(\mathcal{V})$ and $\pi(\mathcal{W})$ separate $\pi(i)$ and $\pi(j)$ in the quotient space $\operatorname{Imm}_{\text {prop }}(M, N) / \operatorname{Diff}(M)$.

(13) Claim. For a proper immersion $i: M \rightarrow N$ and $x \in i(M)$ let $\delta(x) \in \mathbb{N}$ be the number of points in $i^{-1}(x)$. Then $\delta: i(M) \rightarrow \mathbb{N}$ is upper semicontinuous, i.e., the set $\{x \in i(M): \delta(x) \leq k\}$ is open in $i(M)$ for each $k$.

Let $x \in i(M)$ with $\delta(x)=k$ and let $i^{-1}(x)=\left\{y_{1}, \ldots, y_{k}\right\}$. Then there are pairwise disjoint open neighborhoods $W_{n}$ of $y_{n}$ in $M$ such that $\left.i\right|_{W_{n}}$ is an embedding for each $n$. The set $M \backslash\left(\bigcup_{n} W_{n}\right)$ is closed in $M$, and since $i$ is proper the set $i\left(M \backslash\left(\bigcup_{n} W_{n}\right)\right)$ is also closed in $i(M)$ and does not contain $x$. So there is an open neighborhood $U$ of $x$ in $i(M)$ which does not meet $i\left(M \backslash\left(\bigcup_{n} W_{n}\right)\right)$. Obviously $\delta(z) \leq k$ for all $z \in U$.

(14) Claim. Consider two proper immersions $i_{1}$ and $i_{2} \in \operatorname{Imm}_{\text {prop }}(M, N)$ such that $i_{1}(M)=i_{2}(M)=: L \subseteq N$. Then we have mappings $\delta_{1}, \delta_{2}: L \rightarrow \mathbb{N}$ as in (13). If $\delta_{1} \neq \delta_{2}$ then the projections $\pi\left(i_{1}\right)$ and $\pi\left(i_{2}\right)$ are different and can be separated by disjoint open neighborhoods in $\operatorname{Imm}_{\text {prop }}(M, N) / \operatorname{Diff}(M)$.

Let us suppose that $m_{1}=\delta_{1}\left(y_{0}\right) \neq \delta_{2}\left(y_{0}\right)=m_{2}$. There is a small connected open neighborhood $V$ of $y_{0}$ in $N$ such that $i_{1}^{-1}(V)$ has $m_{1}$ connected components and $i_{2}^{-1}(V)$ has $m_{2}$ connected components. This assertions describe Whitney $C^{0}$-open neighborhoods in $\operatorname{Imm}_{\text {prop }}(M, N)$ of $i_{1}$ and $i_{2}$ which are closed under the action of $\operatorname{Diff}(M)$, respectively. Obviously these two neighborhoods are disjoint.

(15) Assumption. We assume that we are given two immersions $i_{1}$ and $i_{2} \in \operatorname{Imm}_{\text {prop }}(M, N)$ with $i_{1}(M)=i_{2}(M)=: L$ such that the functions from (14) are equal: $\delta_{1}=\delta_{2}=: \delta$.

Let $\left(L_{\beta}\right)_{\beta \in B}$ be the partition of $L$ consisting of all pathwise connected components of level sets $\{x \in L: \delta(x)=c\}, c$ some constant. 
Let $B_{0}$ denote the set of all $\beta \in B$ such that the interior of $L_{\beta}$ in $L$ is not empty. Since $M$ is second countable, $B_{0}$ is countable.

(16) Claim. $\bigcup_{\beta \in B_{0}} L_{\beta}$ is dense in $L$.

Let $k_{1}$ be the smallest number in $\delta(L)$ and let $B_{1}$ be the set of all $\beta \in B$ such that $\delta\left(L_{\beta}\right)=k_{1}$. Then by claim (13) each $L_{\beta}$ for $\beta \in B_{1}$ is open. Let $L^{1}$ be the closure of $\bigcup_{\beta \in B_{1}} L_{\beta}$. Let $k_{2}$ be the smallest number in $\delta\left(L \backslash L^{1}\right)$ and let $B_{2}$ be the set of all $\beta \in B$ with $\beta\left(L_{\beta}\right)=k_{2}$ and $L_{\beta} \cap\left(L \backslash L^{1}\right) \neq \emptyset$. Then by claim (13) again $L_{\beta} \cap\left(L \backslash L^{1}\right) \neq \emptyset$ is open in $L$ so $L_{\beta}$ has non empty interior for each $\beta \in B_{2}$. Then let $L^{2}$ denote the closure of $\bigcup_{\beta \in B_{1} \cup B_{2}} L_{\beta}$ and continue the process. If $\delta(L)$ is bounded, the process stops. If $\delta(L)$ is unbounded, by claim (13) we always find new $L_{\beta}$ with non empty interior, we finally exhaust $L$ and claim (16) follows.

Let $\left(M_{\lambda}^{1}\right)_{\lambda \in C^{1}}$ be a suitably chosen cover of $M$ by subsets of the sets $i_{1}^{-1}\left(L_{\beta}\right)$ such that:

(i) Each $\left.i_{1}\right|_{\text {int } M_{\lambda}^{1}}$ is an embedding for each $\lambda$.

(ii) The set $C_{0}^{1}$ of all $\lambda$ with $M_{\lambda}^{1}$ having non empty interior is at most countable. Let $\left(M_{\mu}^{2}\right)_{\mu \in C^{2}}$ be a cover chosen in a similar way for $i_{2}$.

(iii) For each pair $(\mu, \lambda) \in C_{0}^{2} \times C_{0}^{1}$ the two open sets $i_{2}\left(\operatorname{int}\left(M_{\mu}^{2}\right)\right)$ and $i_{1}\left(\operatorname{int}\left(M_{\lambda}^{1}\right)\right)$ in $L$ are either equal or disjoint.

Note that the union $\bigcup_{\lambda \in C_{0}^{1}}$ int $M_{\lambda}^{1}$ is dense in $M$ and thus $\bigcup_{\lambda \in C_{0}^{1}} \overline{M_{\lambda}^{1}}=M$; similarly for the $M_{\mu}^{2}$.

(17) Procedure. Given immersions $i_{1}$ and $i_{2}$ as in (15) we will try to construct a diffeomorphism $f: M \rightarrow M$ with $i_{2} \circ f=i_{1}$. If we meet obstacles to the construction this will give enough control on the situation to separate $i_{1}$ from $i_{2}$.

Choose $\lambda_{0} \in C_{0}^{1}$; so $\operatorname{int} M_{\lambda_{0}}^{1} \neq \emptyset$. Then $i_{1}: \operatorname{int} M_{\lambda_{0}}^{1} \rightarrow L_{\beta_{1}\left(\lambda_{0}\right)}$ is an embedding, where $\beta_{1}: C^{1} \rightarrow B$ is the mapping satisfying $i_{1}\left(M_{\lambda}^{1}\right) \subseteq L_{\beta_{1}(\lambda)}$ for all $\lambda \in C^{1}$.

We choose $\mu_{0} \in \beta_{2}^{-1} \beta_{1}\left(\lambda_{0}\right) \subset C_{0}^{2}$ such that $f:=\left.\left(\left.i_{2}\right|_{\operatorname{int} M_{\mu_{0}}^{2}}\right)^{-1} \circ i_{1}\right|_{\operatorname{int} M_{\lambda_{0}}^{1}}$ is a diffeomorphism int $M_{\lambda_{0}}^{1} \rightarrow \operatorname{int} M_{\mu_{0}}^{2}$; this follows from (iii). Note that $f$ is uniquely determined by the choice of $\mu_{0}$, if it exists, by claim (8). So we will repeat the following construction for every $\mu_{0} \in \beta_{2}^{-1} \beta_{1}\left(\lambda_{0}\right) \subset C_{0}^{2}$.

Now we try to extend $f$. We choose $\lambda_{1} \in C_{0}^{1}$ such that $\bar{M}_{\lambda_{0}}^{1} \cap \bar{M}_{\lambda_{1}}^{1} \neq \emptyset$. Case a. Only $\lambda_{1}=\lambda_{0}$ is possible. So $M_{\lambda_{0}}^{1}$ is dense in $M$ since $M$ is connected and we may extend $f$ by continuity to a diffeomorphism $f: M \rightarrow M$ with $i_{2} \circ f=i_{1}$.

Case b. We can find $\lambda_{1} \neq \lambda_{0}$. We choose $x \in \bar{M}_{\lambda_{0}}^{1} \cap \bar{M}_{\lambda_{1}}^{1}$ and a sequence $\left(x_{n}\right)$ in $M_{\lambda_{0}}^{1}$ with $x_{n} \rightarrow x$. Then we have a sequence $\left(f\left(x_{n}\right)\right)$ in $M$.

Case ba. $y:=\lim f\left(x_{n}\right)$ exists in $M$. Then there is $\mu_{1} \in C_{0}^{2}$ such that $y \in \bar{M}_{\mu_{0}}^{2} \cap \bar{M}_{\mu_{1}}^{2}$.

Let $U_{\alpha_{1}}^{1}$ be an open neighborhood of $x$ in $M$ such that $i_{1} \mid U_{\alpha_{1}}^{1}$ is an embedding and let similarly $U_{\alpha_{2}}^{2}$ be an open neighborhood of $y$ in $M$ such that 
$i_{2} \mid U_{\alpha_{2}}^{2}$ is an embedding. We consider now the set $i_{2}^{-1} i_{1}\left(U_{\alpha_{1}}^{1}\right)$. There are two cases possible.

Case baa. The set $i_{2}^{-1} i_{1}\left(U_{\alpha_{1}}^{1}\right)$ is a neighborhood of $y$. Then we extend $f$ to $i_{1}^{-1}\left(i_{1}\left(U_{\alpha_{1}}^{1}\right) \cap i_{2}\left(U_{\alpha_{2}}^{2}\right)\right)$ by $i_{2}^{-1} \circ i_{1}$. Then $f$ is defined on some open subset of int $M_{\lambda_{1}}^{1}$ and by the situation chosen in (15) and by (iii), the diffeomorphism $f$ extends to the whole of $\operatorname{int} M_{\lambda_{1}}^{1}$.

Case bab. The set $i_{2}^{-1} i_{1}\left(U_{\alpha_{1}}^{1}\right)$ is not a neighborhood of $y$. This is a definite obstruction to the extension of $f$.

Case bb. The sequence $\left(x_{n}\right)$ has no limit in $M$. This is a definite obstruction to the extension of $f$.

If we meet an obstruction we stop and try another $\mu_{0}$. If for all admissible $\mu_{0}$ we meet obstructions we stop and remember the data. If we do not meet an obstruction we repeat the construction with some obvious changes.

(18) Claim. The construction of (17) in the setting of (15) either produces a diffeomorphism $f: M \rightarrow M$ with $i_{2} \circ f=i_{1}$ or we may separate $i_{1}$ and $i_{2}$ by open sets in $\operatorname{Imm}_{\text {prop }}(M, N)$ which are saturated with respect to the action of $\operatorname{Diff}(M)$

If for some $\mu_{0}$ we do not meet any obstruction in the construction (17), the resulting $f$ is defined on the whole of $M$ and it is a continuous mapping $M \rightarrow M$ with $i_{2} \circ f=i_{1}$. Since $i_{1}$ and $i_{2}$ are locally embeddings, $f$ is smooth and of maximal rank. Since $i_{1}$ and $i_{2}$ are proper, $f$ is proper. So the image of $f$ is open and closed and since $M$ is connected, $f$ is a surjective local diffeomorphism, thus a covering mapping $M \rightarrow M$. But since $\delta_{1}=\delta_{2}$ the mapping $f$ must be a 1 -fold covering, i.e., a diffeomorphism.

If for all $\mu_{0} \in \beta_{2}^{-1} \beta_{1}\left(\lambda_{0}\right) \subset C_{0}^{2}$ we meet obstructions we choose small mutually distinct open neighborhoods $V_{\lambda}^{1}$ of the sets $i_{1}\left(M_{\lambda}^{1}\right)$. We consider the Whitney $C^{0}$-open neighborhood $\mathcal{V}_{1}$ of $i_{1}$ consisting of all immersions $j_{1}$ with $j_{1}\left(M_{\lambda}^{1}\right) \subset V_{\lambda}^{1}$ for all $\lambda$. Let $\mathcal{V}_{2}$ be a similar neighborhood of $i_{2}$.

We claim that $\mathcal{V}_{1} \circ \operatorname{Diff}(M)$ and $\mathcal{V}_{2} \circ \operatorname{Diff}(M)$ are disjoint. For that it suffices to show that for any $j_{1} \in \mathcal{V}_{1}$ and $j_{2} \in \mathcal{V}_{2}$ there does not exist a diffeomorphism $f \in \operatorname{Diff}(M)$ with $j_{2} \circ f=j_{1}$. For that to be possible the immersions $j_{1}$ and $j_{2}$ must have the same image $L$ and the same functions $\delta\left(j_{1}\right), \delta\left(j_{2}\right): L \rightarrow \mathbb{N}$. But now the combinatorial relations of the slightly distinct new sets $M_{\lambda}^{1}, L_{\beta}$, and $M_{\mu}^{2}$ are contained in the old ones, so any try to construct such a diffeomorphism $f$ starting from the same $\lambda_{0}$ meets the same obstructions.

Statements (2) and (3) of the theorem are now proved.

(19) Claim. For a non-free immersion $i \in \operatorname{Imm}(M, N)$, the nontrivial isotropy subgroup $\operatorname{Diff}(M)_{i}=\{f \in \operatorname{Diff}(M): i \circ f=i\}$ acts properly discontinuously on $M$, so the projection $q_{1}: M \rightarrow M_{1}:=M / \operatorname{Diff}(M)_{i}$ is a covering map onto a smooth manifold on $M_{1}$. There is an immersion $i_{1}: M_{1} \rightarrow N$ with $i=i_{1} \circ q_{1}$. In particular $\operatorname{Diff}(M)_{i}$ is countable, and is finite if $M$ is compact. 
We have to show that for each $x \in M$ there is an open neighborhood $U$ such that $f(U) \cap U=\emptyset$ for $f \in \operatorname{Diff}(M)_{i} \backslash\{I d\}$. We consider the setup (7) for $i$. By the proof of (8) we have $f\left(U_{\alpha}^{i}\right) \cap U_{\alpha}^{i}=\left\{x \in U_{\alpha}^{i}: f(x)=x\right\}$ for any $f \in \operatorname{Diff}(M)_{i}$. If $f$ has a fixed point then $f=\operatorname{Id}$, by (8), so $f\left(U_{\alpha}^{i}\right) \cap U_{\alpha}^{i}=\emptyset$ for all $f \in \operatorname{Diff}(M)_{i} \backslash\{I d\}$. The rest is clear.

The factorized immersion $i_{1}$ is in general not a free immersion. The following is an example for that: Let $M_{0} \stackrel{\alpha}{\longrightarrow} M_{1} \stackrel{\beta}{\longrightarrow} M_{2} \stackrel{\gamma}{\longrightarrow} M_{3}$ be a sequence of covering maps with fundamental groups $1 \rightarrow G_{1} \rightarrow G_{2} \rightarrow G_{3}$. Then the group of deck transformations of $\gamma$ is given by $\mathcal{N}_{G_{3}}\left(G_{2}\right) / G_{2}$, the normalizer of $G_{2}$ in $G_{3}$, and the group of deck transformations of $\gamma \circ \beta$ is $\mathcal{N}_{G_{3}}\left(G_{1}\right) / G_{1}$. We can easily arrange that $\mathcal{N}_{G_{3}}\left(G_{2}\right) \nsubseteq \mathcal{N}_{G_{3}}\left(G_{1}\right)$, then $\gamma$ admits deck transformations which do not lift to $M_{1}$. Then we thicken all spaces to manifolds, so that $\gamma \circ \beta$ plays the role of the immersion $i$.

(20) Claim. Let $i \in \operatorname{Imm}(M, N)$ be an immersion which is not free. Then there is a submersive covering map $q_{2}: M \rightarrow M_{2}$ such that $i$ factors to an immersion $i_{2}: M_{2} \rightarrow N$ which is free.

Let $q_{0}: M_{0} \rightarrow M$ be the universal covering of $M$ and consider the immersion $i_{0}=i \circ q_{0}: M_{0} \rightarrow N$ and its isotropy group $\operatorname{Diff}\left(M_{0}\right)_{i_{0}}$. By (19) it acts properly discontinuously on $M_{0}$ and we have a submersive covering $q_{02}: M_{0} \rightarrow M_{2}$ and an immersion $i_{2}: M_{2} \rightarrow N$ with $i_{2} \circ q_{02}=i_{0}=i \circ q_{0}$. By comparing the respective groups of deck transformations it is easily seen that $q_{02}: M_{0} \rightarrow M_{2}$ factors over $q_{1} \circ q_{0}: M_{0} \rightarrow M \rightarrow M_{1}$ to a covering $q_{12}: M_{1} \rightarrow M_{2}$. The mapping $q_{2}:=q_{12} \circ q_{1}: M \rightarrow M_{2}$ is the looked for covering: If $f \in \operatorname{Diff}\left(M_{2}\right)$ fixes $i_{2}$, it lifts to a diffeomorphism $f_{0} \in \operatorname{Diff}\left(M_{0}\right)$ which fixes $i_{0}$, so $f_{0} \in \operatorname{Diff}\left(M_{0}\right)_{i_{0}}$, so $f=\mathrm{Id}$.

Statement (4) of the theorem follows from (19) and (20).

(21) Convention. In order to avoid complications we assume now that $M$ is such a manifold that

- For any covering $M \rightarrow M_{1}$, any diffeomorphism $M_{1} \rightarrow M_{1}$ admits a lift $M \rightarrow M$.

If $M$ is simply connected, this condition is satisfied. Also for $M=S^{1}$ the condition is easily seen to be valid. So what follows is applicable to loop spaces.

Condition (21) implies that in the proof of claim (20) we have $M_{1}=M_{2}$.

(22) Description of a neighborhood of a singular orbit. Let $M$ be a manifold satisfying (21). In the situation of (19) we consider the normal bundles $p_{i}: \mathcal{N}(i) \rightarrow M$ and $p_{i_{1}}: \mathcal{N}\left(i_{1}\right) \rightarrow M_{1}$. Then the covering map $q_{1}: M \rightarrow M_{1}$ lifts uniquely to a vector bundle homomorphism $\mathcal{N}\left(q_{1}\right)$ : $\mathcal{N}(i) \rightarrow \mathcal{N}\left(i_{1}\right)$ which is also a covering map, such that $\tau^{i_{1}} \circ \mathcal{N}\left(q_{1}\right)=\tau^{i}$.

We have $M_{1}=M / \operatorname{Diff}(M)_{i}$ and the group $\operatorname{Diff}(M)_{i}$ acts also as the group of deck transformations of the covering $\mathcal{N}\left(q_{1}\right): \mathcal{N}(i) \rightarrow \mathcal{N}\left(i_{1}\right)$ by 
$\operatorname{Diff}(M)_{i} \ni f \mapsto \mathcal{N}(f)$, where

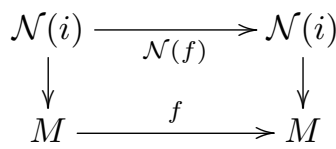

is a vector bundle isomorphism for each $f \in \operatorname{Diff}(M)_{i}$; see the end of (7). If we equip $\mathcal{N}(i)$ and $\mathcal{N}\left(i_{1}\right)$ with the fiber Riemann metrics induced from the fixed Riemannian metric $g$ on $N$, the mappings $\mathcal{N}\left(q_{1}\right)$ and all $\mathcal{N}(f)$ are fiberwise linear isometries.

Let us now consider the right action of $\operatorname{Diff}(M)_{i}$ on the space of sections $\Gamma_{c}(M \leftarrow \mathcal{N}(i))$ given by $f^{*} s:=\mathcal{N}(f)^{-1} \circ s \circ f$.

From the proof of claim (10) we recall now the sets

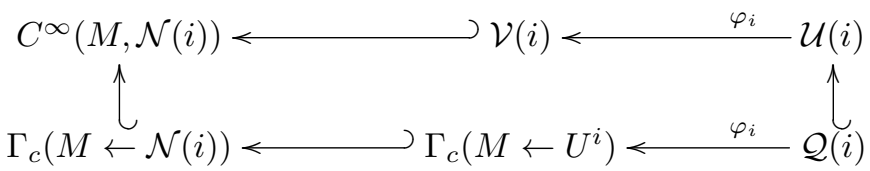

Both mappings $\varphi_{i}$ are diffeomorphisms. But since the action of Diff $(M)$ on $i$ is not free we cannot extend the splitting submanifold $\mathcal{Q}(i)$ to an orbit cylinder as we did in the proof of claim $(10) . \mathcal{Q}(i)$ is a smooth transversal for the orbit though $i$.

For any $f \in \operatorname{Diff}(M)$ and $s \in \Gamma_{c}\left(M \leftarrow U^{i}\right) \subset \Gamma_{c}(M \leftarrow \mathcal{N}(i))$ we have

$$
\varphi_{i}^{-1}\left(f^{*} s\right)=\tau_{*}^{i}\left(f^{*} s\right)=\tau_{*}^{i}(s) \circ f .
$$

So the space $q_{1}^{*} \Gamma_{c}\left(M \leftarrow \mathcal{N}\left(i_{1}\right)\right)$ of all sections of $\mathcal{N}(i) \rightarrow M$ which factor to sections of $\mathcal{N}\left(i_{1}\right) \rightarrow M_{1}$, is exactly the space of all fixed points of the action of $\operatorname{Diff}(M)_{i}$ on $\Gamma_{c}(M \leftarrow \mathcal{N}(i))$; and they are mapped by $\tau_{*}^{i}=\varphi_{i}^{-1}$ to such immersions in $\mathcal{Q}(i)$ which have again $\operatorname{Diff}(M)_{i}$ as isotropy group.

If $s \in \Gamma_{c}\left(M \leftarrow U^{i}\right) \subset \Gamma_{c}(M \leftarrow \mathcal{N}(i))$ is an arbitrary section, the orbit through $\tau_{*}^{i}(s) \in \mathcal{Q}(i)$ hits the transversal $\mathcal{Q}(i)$ again in the points $\tau_{*}^{i}\left(f^{*} s\right)$ for $f \in \operatorname{Diff}(M)_{i}$.

Statement (5) of the theorem is now proved.

(23) The orbit structure. We have the following description of the orbit structure near $i$ in $\operatorname{Imm}(M, N)$ : For fixed $f \in \operatorname{Diff}(M)_{i}$ the set of fixed points $\operatorname{Fix}(f):=\{j \in \mathcal{Q}(i): j \circ f=j\}$ is called a generalized wall. The union of all generalized walls is called the diagram $\mathcal{D}(i)$ of $i$. A connected component of the complement $\mathcal{Q}(i) \backslash \mathcal{D}(i)$ is called a generalized Weyl chamber. The group $\operatorname{Diff}(M)_{i}$ maps walls to walls and chambers to chambers. The immersion $i$ lies in every wall. We shall see shortly that there is only one chamber and that the situation is rather distinct from that of reflection groups.

If we view the diagram in the space $\Gamma_{c}\left(M \leftarrow U^{i}\right) \subset \Gamma_{c}(M \leftarrow \mathcal{N}(i))$ which is diffeomorphic to $\mathcal{Q}(i)$, then it consists of traces of closed linear subspaces, because the action of $\operatorname{Diff}(M)_{i}$ on $\Gamma_{c}(M \leftarrow \mathcal{N}(i))$ consists of linear isometries in the following way. Let us tensor the vector bundle $\mathcal{N}(i) \rightarrow M$ with the natural line bundle of half densities on $M$, and let us remember one positive 
half density to fix an isomorphism with the original bundle. Then $\operatorname{Diff}(M)_{i}$ still acts on this new bundle $\mathcal{N}_{1 / 2}(i) \rightarrow M$ and the pullback action on sections with compact support is isometric for the inner product

$$
\left\langle s_{1}, s_{2}\right\rangle:=\int_{M} g\left(s_{1}, s_{2}\right) .
$$

We now extend the walls and chambers from

$$
\mathcal{Q}(i)=\Gamma_{c}\left(M \leftarrow U^{i}\right) \subset \Gamma_{c}(M \leftarrow \mathcal{N}(i))
$$

to the whole space $\Gamma_{c}(M \leftarrow \mathcal{N}(i))=\Gamma_{c}\left(M \leftarrow \mathcal{N}_{1 / 2}(i)\right)$; recall from (22) that $\operatorname{Diff}(M)_{i}$ acts on the whole space.

(24) Claim. Each wall in $\Gamma_{c}\left(M \leftarrow \mathcal{N}_{1 / 2}(i)\right)$ is a closed linear subspace of infinite codimension. Since there are at most countably many walls, there is only one chamber.

From the proof of claim (19) we know that $f\left(U_{\alpha}^{i}\right) \cap U_{\alpha}^{i}=\emptyset$ for all $f \in$ $\operatorname{Diff}(M)_{i}$ and all sets $U_{\alpha}^{i}$ from the setup (7). Take a section $s$ in the wall of fixed points of $f$. Choose a section $s_{\alpha}$ with support in some $U_{\alpha}^{i}$ and let the section $s$ be defined by $\left.s\right|_{U_{\alpha}^{i}}=\left.s_{\alpha}\right|_{U_{\alpha}^{i}},\left.s\right|_{f^{-1}\left(U_{\alpha}^{i}\right)}=-f^{*} s_{\alpha}, 0$ elsewhere. Then obviously $\left\langle s, s^{\prime}\right\rangle=0$ for all $s^{\prime}$ in the wall of $f$. But this construction furnishes an infinite dimensional space contained in the orthogonal complement of the wall of $f$.

(25) The action of $\operatorname{Diff}(M)$ on $\operatorname{Imm}(M, N) \times M$. Proof of (6).

Here we will consider the right action $(i, x) . f=\left(i \circ f, f^{-1}(x)\right)$ of $\operatorname{Diff}(M)$ on $\operatorname{Imm}(M, N) \times M$. This action is free: If $\left(i \circ f, f^{-1}(x)\right)=(i, x)$ then from claim (8) we get $f=I d_{M}$.

Claim. Let $(i, x) \in \operatorname{Imm}(M, N) \times M$. Then there is an open neighborhood $\mathcal{W}(i, x)$ in $\operatorname{Imm}(M, N) \times M$ which is saturated for the $\operatorname{Diff}(M)$-action and which splits smoothly as

$$
\mathcal{W}(i, x)=\mathcal{Q}(i, x) \times \operatorname{Diff}(M) .
$$

Here $\mathcal{Q}(i, x)$ is a smooth splitting submanifold of $\operatorname{Imm}(M, N) \times M$, diffeomorphic to an open neighborhood of $(0, x)$ in $C^{\infty}(\mathcal{N}(i))$.

Let $\pi: \operatorname{Imm}(M, N) \times M \rightarrow(\operatorname{Imm}(M, N) \times M) / \operatorname{Diff}(M)=S(M, N)$ be the projection onto the orbit space, which we equip with the quotient topology. Then $\left.\pi\right|_{\mathcal{Q}(i, x)}: \mathcal{Q}(i, x) \rightarrow \pi(\mathcal{Q}(i, x))$ is bijective onto an open subset of the quotient. If $(i, x)$ runs through $\operatorname{Imm}(M, N) \times M$ these mappings define a smooth atlas for the quotient space, so that

$$
(\operatorname{Imm}(M, N) \times M, \pi,(\operatorname{Imm}(M, N) \times M) / \operatorname{Diff}(M), \operatorname{Diff}(M))
$$

is a smooth principal fiber bundle with structure group $\operatorname{Diff}(M)$.

If we restrict to the open subset $\operatorname{Imm}_{\text {prop }}(M, N) \times M$ of proper immersions times $M$ then the base space is Hausdorff.

By claim (19), the isotropy subgroup $\operatorname{Diff}(M)_{i}=\{f \in \operatorname{Diff}(M): i \circ f=i\}$ acts properly discontinuously on $M$, so $q_{1}: M \rightarrow M / \operatorname{Diff}(M)_{i}=: M_{1}$ is a covering. We choose an open neighborhood $W_{x}$ of $x$ in $M$ such that $q_{1}$ : $W_{x} \rightarrow M_{1}$ is injective. 
Now we adapt the second half of the proof of claim (10) and use freely all the notation from there. We consider the open set

$$
\begin{aligned}
\left\{\left(h \circ f, f^{-1}(y)\right): h \in \mathcal{V}(i), y \in\right. & \left.W_{x}, f \in \operatorname{Diff}(M)\right\} \subset \\
& \subset C^{\infty}\left(M, U^{i}\right) \times M \subset C^{\infty}(M, \mathcal{N}(i)) \times M .
\end{aligned}
$$

We have a smooth mapping from it into $\Gamma_{c}\left(M \leftarrow U^{i}\right) \times W_{x} \times \operatorname{Diff}(M)$ which is given by $(h, y) \mapsto\left(h \circ(p \circ h)^{-1},(p \circ h)(y), p \circ h\right)$, where $\Gamma_{c}\left(M \leftarrow U^{i}\right)$ is the space of sections with compact support of $U^{i} \rightarrow M$. We now put

$$
\mathcal{Q}(i, x):=\tau_{*}^{i}\left(\Gamma_{c}\left(M \leftarrow U^{i}\right) \cap \mathcal{V}(i)\right) \times W_{x} \subset \operatorname{Imm}(M, N) \times M .
$$

Then we have

$$
\begin{aligned}
\mathcal{W}(i, x): & =\left\{(h \circ f, f(y)): h \in \mathcal{U}(i), y \in W_{x}, f \in \operatorname{Diff}(M)\right\} \\
& \cong \mathcal{Q}(i, x) \times \operatorname{Diff}(M) \cong\left(\Gamma_{c}\left(M \leftarrow U^{i}\right) \cap \mathcal{V}(i)\right) \times W_{x} \times \operatorname{Diff}(M),
\end{aligned}
$$

since the action of $\operatorname{Diff}(M)$ is free. The quotient mapping $\left.\pi\right|_{\mathcal{Q}(i)}: \mathcal{Q}(i) \rightarrow$ $\operatorname{Imm}_{\text {free }}(M, N) / \operatorname{Diff}(M)$ is bijective onto an open set in the quotient. We now use $\left(\varphi_{i} \times I d_{W_{x}}\right) \circ\left(\left.\pi\right|_{\mathcal{Q}(i, x)}\right)^{-1}: \pi(\mathcal{Q}(i, x)) \rightarrow \Gamma_{c}\left(M \leftarrow U^{i}\right) \times W_{x}$ as a chart for the quotient space. In order to investigate the chart change let $(j, y) \in \operatorname{Imm}(M, N) \times M$ be such that $\pi(\mathcal{Q}(i, x)) \cap \pi(\mathcal{Q}(j, y)) \neq \emptyset$. Then there exists $(h, z) \in \mathcal{W}(i, x) \cap \mathcal{Q}(j, y)$, so there exists a unique $f \in \operatorname{Diff}(M)$ (given by $f=p \circ \varphi_{i}(h)$ ) such that $\left(h \circ f^{-1}, f(z)\right) \in \mathcal{Q}(i, x)$. If we consider $\left(j \circ f^{-1}, f(y)\right)$ instead of $(j, y)$ and call it again $(j, y)$, we have $\mathcal{Q}(i, x) \cap$ $\mathcal{Q}(j, y) \neq \emptyset$ and consequently $\mathcal{U}(i) \cap \mathcal{U}(j) \neq \emptyset$. Now the first component of the chart change is smooth by the argument in the end of the proof of claim (10), and the second component is just $I d_{W_{x} \cap W_{y}}$.

The result about Hausdorff follows from claim (11). The fibers over $\operatorname{Imm}(M, N) / \operatorname{Diff}(M)$ can be read off the following diagram:

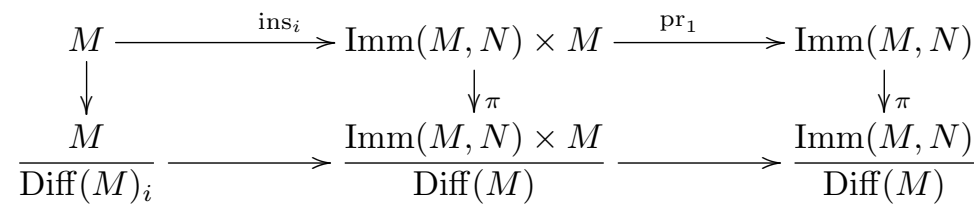

This finishes the proof of Theorem 7.3 .

\section{WEAK RIEMANNIAN MANIFOLDS}

If an infinite dimensional manifold is not modeled on a Hilbert space, then a Riemannian metric cannot describe the topology on each tangent space. We have to deal with more complicated situations.

8.1. Manifolds, vector fields, differential forms. Let $M$ be a smooth manifold modeled on convenient vector spaces. Tangent vectors to $M$ are kinematic ones.

The reason for this is that eventually we want flows of vector fields, and that there are too many derivations in infinite dimensions, even on a Hilbert 
space $H$ : Let $\alpha \in L(H, H)$ be a continuous linear functional which vanishes on the subspace of compact operators, thus also on $H \otimes H$. Then the linear functional $f \mapsto \alpha\left(d^{2} f(0)\right)$ is a derivation at 0 on $C^{\infty}(H)$, since

$\alpha\left(d^{2}(f . g)(0)\right)=\alpha\left(d^{2} f(0) \cdot g(0)+d f(0) \otimes d g(0)+d g(0) \otimes d f(0)+f(0) \cdot d^{2} g(0)\right)$

and $\alpha$ vanishes on the two middle terms. There are even non-zero derivations which differentiate 3 times, see [55, 28.4].

The (kinematic) tangent bundle $T M$ is then a smooth vector bundle as usual. Differential forms of degree $k$ are then smooth sections of the bundle $L_{\text {skew }}^{k}(T M ; \mathbb{R})$ of skew symmetric $k$-linear functionals on the tangent bundle, since this is the only version which admits exterior derivative, Lie derivatives along vector field, and pullbacks along arbitrary smooth mappings; see [55, 33.21]. The de Rham cohomology equals singular cohomology with real coefficients if the manifold is smoothly paracompact; see [71] and [55, Section 34]. If a vector field admits a flow, then each integral curve is uniquely given as a flow line; see [55, 32.14].

8.2. Weak Riemannian manifolds. Let $M$ be a smooth manifold modeled on convenient locally convex vector spaces. A smooth Riemannian metric $g$ on $M$ is called weak if $g_{x}: T_{x} M \rightarrow T_{x}^{*} M$ is only injective for each $x \in M$. The image $g(T M) \subset T^{*} M$ is called the smooth cotangent bundle associated to $g$. Then $g^{-1}$ is the metric on the smooth cotangent bundle as well as the morphism $g(T M) \rightarrow T M$. We have a special class of 1 -forms $\Omega_{g}^{1}(M):=$ $\Gamma(g(T M))$ for which the musical mappings make sense: $\alpha^{\sharp}=g^{-1} \alpha \in \mathfrak{X}(M)$ and $X^{b}=g X$. These 1 -forms separate points on $T M$. The exterior derivative is defined by $d: \Omega_{g}^{1}(M) \rightarrow \Omega^{2}(M)=\Gamma\left(L_{\text {skew }}^{2}(T M ; \mathbb{R})\right)$ since the embedding $g(T M) \subset T^{*} M$ is a smooth fiber linear mapping.

Existence of the Levi-Civita covariant derivative is equivalent to: The metric itself admits symmetric gradients with respect to itself. Locally this means: If $M$ is $c^{\infty}$-open in a convenient vector space $V_{M}$. Then:

$$
D_{x, X} g_{x}(X, Y)=g_{x}\left(X, \operatorname{grad}_{1} g(x)(X, Y)\right)=g_{x}\left(\operatorname{grad}_{2} g(x)(X, X), Y\right)
$$

where $D_{x, X}$ denote the directional derivative at $x$ in the direction $X$, and where the mappings $\operatorname{grad}_{1} g$ and sym $\operatorname{grad}_{2} g: M \times V_{M} \times V_{M} \rightarrow V_{M}$, given by $(x, X) \mapsto \operatorname{grad}_{1,2} g(x)(X, X)$, are smooth and quadratic in $X \in V_{M}$. The geodesic equation then is (again locally) given by

$$
c_{t t}=\frac{1}{2} \operatorname{grad}_{1} g(c)\left(c_{t}, c_{t}\right)-\operatorname{grad}_{2} g(c)\left(c_{t}, c_{t}\right)
$$

This formula corresponds to the usual formula for the geodesic flow using Christoffel symbols, expanded out using the first derivatives of the metric tensor. For the existence of the covariant derivative see [68, 2.4], and for the geodesic equation see [76, 2.1 and 2.4]; there this is done in a special case, but the method works in the general case without changes. See also [12, 4.2, 4.3 , and 4.4] for a derivation in another special case. 
8.3. Weak Riemannian metrics on spaces of immersions. For a compact manifold $M$ and a finite dimensional Riemannian manifold $(N, \bar{g})$ we can consider the following weak Riemannian metrics on the manifold $\operatorname{Imm}(M, N)$ of smooth immersions $M \rightarrow N$ :

$$
\begin{aligned}
& G_{f}^{0}(h, k)=\int_{M} \bar{g}(h, k) \operatorname{vol}\left(f^{*} \bar{g}\right) \quad \text { the } L^{2} \text {-metric, } \\
& G_{f}^{s}(h, k)=\int_{M} \bar{g}\left(\left(1+\Delta^{f^{*} \bar{g}}\right)^{s} h, k\right) \operatorname{vol}\left(f^{*} \bar{g}\right) \quad \text { a Sobolev metric of order } s, \\
& G_{f}^{\Phi}(h, g)=\int_{M} \Phi(f) \bar{g}(h, k) \operatorname{vol}\left(f^{*} \bar{g}\right) \quad \text { an almost local metric. }
\end{aligned}
$$

Here $\operatorname{vol}\left(f^{*} \bar{g}\right)$ is the volume density on $M$ of the pullback metric $g=f^{*} \bar{g}$, and $\Delta^{f^{*} \bar{g}}$ is the (Bochner) Laplacian with respect to $g$ and $\bar{g}$ acting on sections of $f^{*} T N$, and $\Phi(f)$ is a positive function of the total volume $\operatorname{Vol}\left(f^{*} g\right)=$ $\int_{M} \operatorname{vol}\left(f^{*} g\right)$, of the scalar curvature $\operatorname{Scal}\left(f^{*} \bar{g}\right)$, and of the mean curvature $\operatorname{Tr}\left(S^{f}\right), S^{f}$ being the second fundamental form. See [12, [13] for more information. All these metrics are invariant for the right action of the reparameterization group $\operatorname{Diff}(M)$, so they descend to metrics on shape space $B_{i}(M, N)$ (off the singularities) such that the projection $\operatorname{Imm}(M, N) \rightarrow B_{i}(M, N)$ is a Riemannian submersion of a benign type: the $G$-orthogonal component to the tangent space to the $\operatorname{Diff}(M)$-orbit consists always of smooth vector fields. So there is no need to use the notion of robust weak Riemannian metrics discussed below.

8.4. Theorem. The Riemannian metrics on $\operatorname{Imm}(M, N)$ defined in 8.3 have the following properties:

(1) Geodesic distance on $\operatorname{Imm}(M, N)$, defined as the infimum of pathlengths of smooth isotopies between two immersions, vanishes for the $L^{2}$-metric $G^{0}$.

(2) Geodesic distance is positive on $B_{i}(M, N)$ for the almost local metric $G^{\Phi}$ if $\Phi(f) \geq 1+A \operatorname{Tr}\left(S^{F}\right)$, or if $\Phi(f) \geq A \operatorname{Vol}\left(f^{*} \bar{g}\right)$, for some $A>0$.

(3) Geodesic distance is positive on $B_{i}(M, N)$ for the Sobolev metric $G^{s}$ if $s \geq 1$.

(4) The geodesic equation is locally well-posed on $\operatorname{Imm}(M, N)$ for the Sobolev metric $G^{s}$ if $s \geq 1$, and globally well-posed (and thus geodesically complete) on $\operatorname{Imm}\left(S^{1}, \mathbb{R}^{n}\right)$, if $s \geq 2$.

(1) is due to 75 for $B_{i}\left(S^{1}, \mathbb{R}^{2}\right)$, to [74 for $B_{i}(M, N)$ and for $\operatorname{Diff}(M)$, which combines to the result for $\operatorname{Imm}(M, N)$ as noted in 6. (2) is proved in [13. For (3) see [12. (4) is due to [21] and [20].

8.5. Analysis tools on regular Lie groups and on $\operatorname{Diff}(M)$ for a Whitney manifold germ. Let $G$ be a regular convenient Lie group, with Lie algebra $\mathfrak{g}$. We also consider a Frölicher group $G=\operatorname{Diff}(M)$ for a Whitney manifold germ $M \subset \tilde{M}$ with Lie algebra $\mathfrak{g}=\mathfrak{X}_{c, \partial}(M)$, with the negative of the usual Lie bracket, as described in $6.3-6.6$ 
Let $\mu: G \times G \rightarrow G$ be the group multiplication, $\mu_{x}$ the left translation and $\mu^{y}$ the right translation, $\mu_{x}(y)=\mu^{y}(x)=x y=\mu(x, y)$. The adjoint action $\operatorname{Ad}: G \rightarrow G L(\mathfrak{g})$ is given by $\operatorname{Ad}(g) X=T\left(\mu^{g^{-1}}\right) \cdot T\left(\mu_{g}\right) X$. Let $L, R$ : $\mathfrak{g} \rightarrow \mathfrak{X}(G)$ be the left and right invariant vector field mappings, given by $L_{X}(g)=T_{e}\left(\mu_{g}\right) . X$ and $R_{X}=T_{e}\left(\mu^{g}\right) . X$, respectively. They are related by $L_{X}(g)=R_{\operatorname{Ad}(g) X}(g)$. Their flows are given by

$$
\begin{aligned}
& \mathrm{Fl}_{t}^{L_{X}}(g)=g \cdot \exp (t X)=\mu^{\exp (t X)}(g), \\
& \mathrm{Fl}_{t}^{R_{X}}(g)=\exp (t X) \cdot g=\mu_{\exp (t X)}(g) .
\end{aligned}
$$

The right Maurer-Cartan form $\kappa=\kappa^{r} \in \Omega^{1}(G, \mathfrak{g})$ is given by $\kappa_{x}(\xi):=$ $T_{x}\left(\mu^{x^{-1}}\right) \cdot \xi$. It satisfies the left Maurer-Cartan equation $d \kappa^{r}-\frac{1}{2}\left[\kappa^{r}, \kappa^{r}\right] \hat{\mathfrak{g}}=0$, where $[,]^{\wedge}$ denotes the wedge product of $\mathfrak{g}$-valued forms on $G$ induced by the Lie bracket. Note that $\frac{1}{2}\left[\kappa^{r}, \kappa^{r}\right]^{\wedge}(\xi, \eta)=\left[\kappa^{r}(\xi), \kappa^{r}(\eta)\right]$.

Namely, evaluate $d \kappa^{r}$ on right invariant vector fields $R_{X}, R_{Y}$ for $X, Y \in \mathfrak{g}$.

$$
\begin{aligned}
\left(d \kappa^{r}\right)\left(R_{X}, R_{Y}\right) & =R_{X}\left(\kappa^{r}\left(R_{Y}\right)\right)-R_{Y}\left(\kappa^{r}\left(R_{X}\right)\right)-\kappa^{r}\left(\left[R_{X}, R_{Y}\right]\right) \\
& =R_{X}(Y)-R_{Y}(X)+[X, Y]=0-0+\left[\kappa^{r}\left(R_{X}\right), \kappa^{r}\left(R_{Y}\right)\right] .
\end{aligned}
$$

The left Maurer-Cartan form $\kappa^{l} \in \Omega^{1}(G, \mathfrak{g})$ is given by $\kappa_{x}^{l}(\xi):=T_{x}\left(\mu_{x^{-1}}\right) \cdot \xi$. The left Maurer-Cartan form $\kappa^{l}$ satisfies the right Maurer-Cartan equation $d \kappa^{l}+\frac{1}{2}\left[\kappa^{l}, \kappa^{l}\right]_{\mathfrak{g}}^{\wedge}=0$.

The (exterior) derivative of the function $\operatorname{Ad}: G \rightarrow G L(\mathfrak{g})$ satisfies

$$
d \operatorname{Ad}=\left(\operatorname{ad} \circ \kappa^{r}\right) \cdot \operatorname{Ad}=\operatorname{Ad} \cdot\left(\operatorname{ad} \circ \kappa^{l}\right)
$$

since we have

$$
\begin{aligned}
d \operatorname{Ad}\left(T \mu^{g} \cdot X\right) & =\left.\partial_{t}\right|_{0} \operatorname{Ad}(\exp (t X) \cdot g)=\left.\partial_{t}\right|_{0} \operatorname{Ad}(\exp (t X)) \cdot \operatorname{Ad}(g) \\
& =\operatorname{ad}\left(\kappa^{r}\left(T \mu^{g} \cdot X\right)\right) \cdot \operatorname{Ad}(g) \\
d \operatorname{Ad}\left(T \mu_{g} \cdot X\right) & =\left.\partial_{t}\right|_{0} \operatorname{Ad}(g \cdot \exp (t X))=\operatorname{Ad}(g) \cdot \operatorname{ad}\left(\kappa^{l}\left(T \mu_{g} \cdot X\right)\right)
\end{aligned}
$$

8.6. Right invariant weak Riemannian metrics on regular Lie groups and on $\operatorname{Diff}(M)$ for a Whitney manifold germ. We continue under the assumptions of 8.5 , Let $\gamma=\mathfrak{g} \times \mathfrak{g} \rightarrow \mathbb{R}$ be a positive-definite bounded (weak) inner product. Then

$$
\gamma_{x}(\xi, \eta)=\gamma\left(T\left(\mu^{x^{-1}}\right) \cdot \xi, T\left(\mu^{x^{-1}}\right) \cdot \eta\right)=\gamma(\kappa(\xi), \kappa(\eta))
$$

is a right invariant (weak) Riemannian metric on $G$ and any (weak) right invariant bounded Riemannian metric is of this form, for suitable $\gamma$. Denote by $\check{\gamma}: \mathfrak{g} \rightarrow \mathfrak{g}^{*}$ the mapping induced by $\gamma$, from the Lie algebra into its dual (of bounded linear functionals) and by $\langle\alpha, X\rangle_{\mathfrak{g}}$ the duality evaluation between $\alpha \in \mathfrak{g}^{*}$ and $X \in \mathfrak{g}$.

Let $g:[a, b] \rightarrow G$ be a smooth curve. The velocity field of $g$, viewed in the right trivializations, coincides with the right logarithmic derivative

$$
\delta^{r}(g):=T\left(\mu^{g^{-1}}\right) \cdot \partial_{t} g=\kappa\left(\partial_{t} g\right)=\left(g^{*} \kappa\right)\left(\partial_{t}\right) .
$$


The energy of the curve $g(t)$ is given by

$$
E(g)=\frac{1}{2} \int_{a}^{b} \gamma_{g}\left(g^{\prime}, g^{\prime}\right) d t=\frac{1}{2} \int_{a}^{b} \gamma\left(\left(g^{*} \kappa\right)\left(\partial_{t}\right),\left(g^{*} \kappa\right)\left(\partial_{t}\right)\right) d t
$$

For a variation $g(s, t)$ with fixed endpoints we then use that

$$
d\left(g^{*} \kappa\right)\left(\partial_{t}, \partial_{s}\right)=\partial_{t}\left(g^{*} \kappa\left(\partial_{s}\right)\right)-\partial_{s}\left(g^{*} \kappa\left(\partial_{t}\right)\right)-0,
$$

partial integration, and the left Maurer-Cartan equation to obtain

$$
\begin{aligned}
& \partial_{s} E(g)=\frac{1}{2} \int_{a}^{b} 2 \gamma\left(\partial_{s}\left(g^{*} \kappa\right)\left(\partial_{t}\right),\left(g^{*} \kappa\right)\left(\partial_{t}\right)\right) d t \\
& =\int_{a}^{b} \gamma\left(\partial_{t}\left(g^{*} \kappa\right)\left(\partial_{s}\right)-d\left(g^{*} \kappa\right)\left(\partial_{t}, \partial_{s}\right),\left(g^{*} \kappa\right)\left(\partial_{t}\right)\right) d t \\
& =-\int_{a}^{b} \gamma\left(\left(g^{*} \kappa\right)\left(\partial_{s}\right), \partial_{t}\left(g^{*} \kappa\right)\left(\partial_{t}\right)\right) d t \\
& \quad-\int_{a}^{b} \gamma\left(\left[\left(g^{*} \kappa\right)\left(\partial_{t}\right),\left(g^{*} \kappa\right)\left(\partial_{s}\right)\right],\left(g^{*} \kappa\right)\left(\partial_{t}\right)\right) d t \\
& =-\int_{a}^{b}\left\langle\check{\gamma}\left(\partial_{t}\left(g^{*} \kappa\right)\left(\partial_{t}\right)\right),\left(g^{*} \kappa\right)\left(\partial_{s}\right)\right\rangle_{\mathfrak{g}} d t \\
& \quad-\int_{a}^{b}\left\langle\check{\gamma}\left(\left(g^{*} \kappa\right)\left(\partial_{t}\right)\right), \operatorname{ad}_{\left(g^{*} \kappa\right)\left(\partial_{t}\right)}\left(g^{*} \kappa\right)\left(\partial_{s}\right)\right\rangle_{\mathfrak{g}} d t \\
& =-\int_{a}^{b}\left\langle\check{\gamma}\left(\partial_{t}\left(g^{*} \kappa\right)\left(\partial_{t}\right)\right)+\left(\operatorname{ad}_{\left(g^{*} \kappa\right)\left(\partial_{t}\right)}\right)^{*} \check{\gamma}\left(\left(g^{*} \kappa\right)\left(\partial_{t}\right)\right),\left(g^{*} \kappa\right)\left(\partial_{s}\right)\right\rangle_{\mathfrak{g}} d t .
\end{aligned}
$$

Thus the curve $g(0, t)$ is critical for the energy if and only if

$$
\check{\gamma}\left(\partial_{t}\left(g^{*} \kappa\right)\left(\partial_{t}\right)\right)+\left(\operatorname{ad}_{\left(g^{*} \kappa\right)\left(\partial_{t}\right)}\right)^{*} \check{\gamma}\left(\left(g^{*} \kappa\right)\left(\partial_{t}\right)\right)=0 .
$$

In terms of the right logarithmic derivative $u:[a, b] \rightarrow \mathfrak{g}$ of $g:[a, b] \rightarrow G$, given by $u(t):=g^{*} \kappa\left(\partial_{t}\right)=T_{g(t)}\left(\mu^{g(t)^{-1}}\right) \cdot g^{\prime}(t)$, the geodesic equation has the expression

$$
\partial_{t} u=-\check{\gamma}^{-1} \operatorname{ad}(u)^{*} \check{\gamma}(u)
$$

Thus the geodesic equation exists in general if and only if $\operatorname{ad}(X)^{*} \check{\gamma}(X)$ is in the image of $\check{\gamma}: \mathfrak{g} \rightarrow \mathfrak{g}^{*}$, i.e.,

$$
\operatorname{ad}(X)^{*} \check{\gamma}(X) \in \check{\gamma}(\mathfrak{g})
$$

for every $X \in \mathfrak{X}$; this leads to the existence of the Christoffel symbols. Arnold [4] asked for the more restrictive condition $\operatorname{ad}(X)^{*} \check{\gamma}(Y) \in \check{\gamma}(\mathfrak{g})$ for all $X, Y \in$ $\mathfrak{g}$. The geodesic equation for the momentum $p:=\gamma(u)$ is

$$
p_{t}=-\operatorname{ad}\left(\check{\gamma}^{-1}(p)\right)^{*} p .
$$

There are situations, see theorem 8.11 or [9], where only the more general condition is satisfied, but where the usual transpose $\operatorname{ad}^{\top}(X)$ of $\operatorname{ad}(X)$,

$$
\operatorname{ad}^{\top}(X):=\check{\gamma}^{-1} \circ \operatorname{ad}_{X}^{*} \circ \check{\gamma}
$$


does not exist for all $X$.

We describe now the covariant derivative and the curvature. The right trivialization $\left(\pi_{G}, \kappa^{r}\right): T G \rightarrow G \times \mathfrak{g}$ induces the isomorphism $R: C^{\infty}(G, \mathfrak{g}) \rightarrow$ $\mathfrak{X}(G)$, given by $R(X)(x):=R_{X}(x):=T_{e}\left(\mu^{x}\right) \cdot X(x)$, for $X \in C^{\infty}(G, \mathfrak{g})$ and $x \in G$. Here $\mathfrak{X}(G):=\Gamma(T G)$ denotes the Lie algebra of all vector fields. For the Lie bracket and the Riemannian metric we have

$$
\begin{aligned}
{\left[R_{X}, R_{Y}\right] } & =R\left(-[X, Y]_{\mathfrak{g}}+d Y \cdot R_{X}-d X \cdot R_{Y}\right), \\
R^{-1}\left[R_{X}, R_{Y}\right] & =-[X, Y]_{\mathfrak{g}}+R_{X}(Y)-R_{Y}(X), \\
\gamma_{x}\left(R_{X}(x), R_{Y}(x)\right) & =\gamma(X(x), Y(x)), x \in G .
\end{aligned}
$$

In what follows, we shall perform all computations in $C^{\infty}(G, \mathfrak{g})$ instead of $\mathfrak{X}(G)$. In particular, we shall use the convention

$$
\nabla_{X} Y:=R^{-1}\left(\nabla_{R_{X}} R_{Y}\right) \quad \text { for } X, Y \in C^{\infty}(G, \mathfrak{g})
$$

to express the Levi-Civita covariant derivative.

8.7. Lemma. [9, 3.3] Assume that for all $\xi \in \mathfrak{g}$ the element $\operatorname{ad}(\xi)^{*} \check{\gamma}(\xi) \in \mathfrak{g}^{*}$ is in the image of $\check{\gamma}: \mathfrak{g} \rightarrow \mathfrak{g}^{*}$ and that $\xi \mapsto \check{\gamma}^{-1} \operatorname{ad}(\xi)^{*} \check{\gamma}(\xi)$ is bounded quadratic (or, equivalently, smooth). Then the Levi-Civita covariant derivative of the metric $\gamma$ exists and is given for any $X, Y \in C^{\infty}(G, \mathfrak{g})$ in terms of the isomorphism $R$ by

$$
\nabla_{X} Y=d Y \cdot R_{X}+\rho(X) Y-\frac{1}{2} \operatorname{ad}(X) Y,
$$

where

$\rho(\xi) \eta=\frac{1}{4} \check{\gamma}^{-1}\left(\operatorname{ad}_{\xi+\eta}^{*} \check{\gamma}(\xi+\eta)-\operatorname{ad}_{\xi-\eta}^{*} \check{\gamma}(\xi-\eta)\right)=\frac{1}{2} \check{\gamma}^{-1}\left(\operatorname{ad}_{\xi}^{*} \check{\gamma}(\eta)+\operatorname{ad}_{\eta}^{*} \check{\gamma}(\xi)\right)$ is the polarized version. The mapping $\rho: \mathfrak{g} \rightarrow L(\mathfrak{g}, \mathfrak{g})$ is bounded, and we have $\rho(\xi) \eta=\rho(\eta) \xi$. We also have

$$
\begin{gathered}
\gamma(\rho(\xi) \eta, \zeta)=\frac{1}{2} \gamma(\xi, \operatorname{ad}(\eta) \zeta)+\frac{1}{2} \gamma(\eta, \operatorname{ad}(\xi) \zeta) \\
\gamma(\rho(\xi) \eta, \zeta)+\gamma(\rho(\eta) \zeta, \xi)+\gamma(\rho(\zeta) \xi, \xi)=0
\end{gathered}
$$

For $X, Y \in C^{\infty}(G, \mathfrak{g})$ we have

$$
\left[R_{X}, \operatorname{ad}(Y)\right]=\operatorname{ad}\left(R_{X}(Y)\right) \quad \text { and } \quad\left[R_{X}, \rho(Y)\right]=\rho\left(R_{X}(Y)\right) .
$$

The Riemannian curvature is then computed as follows:

$$
\begin{aligned}
\mathcal{R}(X, Y)=\left[\nabla_{X}, \nabla_{Y}\right]-\nabla_{-[X, Y]_{\mathfrak{g}}+R_{X}(Y)-R_{Y}(X)} \\
=\left[R_{X}+\rho_{X}-\frac{1}{2} \operatorname{ad}_{X}, R_{Y}+\rho_{Y}-\frac{1}{2} \operatorname{ad}_{Y}\right] \\
\quad-R\left(-[X, Y]_{\mathfrak{g}}+R_{X}(Y)-R_{Y}(X)\right)-\rho\left(-[X, Y]_{\mathfrak{g}}+R_{X}(Y)-R_{Y}(X)\right) \\
\quad+\frac{1}{2} \operatorname{ad}\left(-[X, Y]_{\mathfrak{g}}+R_{X}(Y)-R_{Y}(X)\right) \\
=\left[\rho_{X}, \rho_{Y}\right]+\rho_{[X, Y]_{\mathfrak{g}}}-\frac{1}{2}\left[\rho_{X}, \operatorname{ad}_{Y}\right]+\frac{1}{2}\left[\rho_{Y}, \operatorname{ad}_{X}\right]-\frac{1}{4} \operatorname{ad}_{[X, Y]_{\mathfrak{g}}}
\end{aligned}
$$

which is visibly a tensor field. 
For the numerator of the sectional curvature we obtain

$$
\begin{aligned}
& \gamma(\mathcal{R}(X, Y) X, Y)=\gamma\left(\rho_{X} \rho_{Y} X, Y\right)-\gamma\left(\rho_{Y} \rho_{X} X, Y\right)+\gamma\left(\rho_{[X, Y]} X, Y\right) \\
& \quad-\frac{1}{2} \gamma\left(\rho_{X}[Y, X], Y\right)+\frac{1}{2} \gamma\left(\left[Y, \rho_{X} X\right], Y\right) \\
&+ 0-\frac{1}{2} \gamma\left(\left[X, \rho_{Y} X\right], Y\right)-\frac{1}{4} \gamma([[X, Y], X], Y) \\
&= \gamma\left(\rho_{X} X, \rho_{Y} Y\right)-\left\|\rho_{X} Y\right\|_{\gamma}^{2}+\frac{3}{4}\|[X, Y]\|_{\gamma}^{2} \\
&-\frac{1}{2} \gamma(X,[Y,[X, Y]])+\frac{1}{2} \gamma(Y,[X,[X, Y]]) \\
&= \gamma\left(\rho_{X} X, \rho_{Y} Y\right)-\left\|\rho_{X} Y\right\|_{\gamma}^{2}+\frac{3}{4}\|[X, Y]\|_{\gamma}^{2} \\
&\left.-\gamma\left(\rho_{X} Y,[X, Y]\right]\right)+\gamma(Y,[X,[X, Y]]) .
\end{aligned}
$$

If the adjoint $\operatorname{ad}(X)^{\top}: \mathfrak{g} \rightarrow \mathfrak{g}$ exists, this is easily seen to coincide with Arnold's original formula 4,

$$
\begin{aligned}
\gamma(\mathcal{R}(X, Y) X, Y)= & -\frac{1}{4}\left\|\operatorname{ad}(X)^{\top} Y+\operatorname{ad}(Y)^{\top} X\right\|_{\gamma}^{2}+\gamma\left(\operatorname{ad}(X)^{\top} X, \operatorname{ad}(Y)^{\top} Y\right) \\
& +\frac{1}{2} \gamma\left(\operatorname{ad}(X)^{\top} Y-\operatorname{ad}(Y)^{\top} X, \operatorname{ad}(X) Y\right)+\frac{3}{4}\|[X, Y]\|_{\gamma}^{2} .
\end{aligned}
$$

8.8. Examples of weak right invariant Riemannian metrics on diffeomorphism groups. Let $M$ be a finite dimensional manifold. We consider the following regular Lie groups: $\operatorname{Diff}(M)$, the group of all diffeomorphisms of $M$ if $M$ is compact. $\operatorname{Diff}_{c}(M)$, the group of diffeomorphisms with compact support, if $M$ is not compact. If $M=\mathbb{R}^{n}$, we also may consider one of the following: Diff $\mathcal{S}\left(\mathbb{R}^{n}\right)$, the group of all diffeomorphisms which fall rapidly to the identity. Diff $W^{\infty, p}\left(\mathbb{R}^{n}\right)$, the group of all diffeomorphisms which are modeled on the space $W^{\infty, p}\left(\mathbb{R}^{n}\right)^{n}$, the intersection of all $W^{k, p}$-Sobolev spaces of vector fields. The last type of groups works also for a Riemannian manifold of bounded geometry $(M, \bar{g})$; see 30] for Sobolev spaces on them. In the following we write $\operatorname{Diff}_{\mathcal{A}}(M)$ for any of these groups. The Lie algebras are the spaces $\mathfrak{X}_{\mathcal{A}}(M)$ of vector fields, where $\mathcal{A} \in\left\{C_{c}^{\infty}, \mathcal{S}, W^{\infty, p}\right\}$, with the negative of the usual bracket as Lie bracket.

Most of the following weak Riemannian metrics also make sense on $\operatorname{Diff}(M)$ for a compact Whitney manifold germ $M \subset \tilde{M}$, but their behavior has not been investigated. In particular, I do not know how the Laplacian $1+\Delta^{g}$ behaves on $\mathfrak{X}_{\partial}(M)$ and its Sobolev completions.

A right invariant weak inner product on $\operatorname{Diff}_{\mathcal{A}}(M)$ is given by a smooth positive-definite inner product $\gamma$ on the Lie algebra $\mathfrak{X}_{\mathcal{A}}(M)$ which is described by the inertia operator $L=\check{\gamma}: \mathfrak{X}_{\mathcal{A}}(M) \rightarrow \mathfrak{X}_{\mathcal{A}}(M)^{\prime}$ and we shall denote its inverse by $K=L^{-1}: L\left(\mathfrak{X}_{\mathcal{A}}(M)\right) \rightarrow \mathfrak{X}_{\mathcal{A}}(M)$. Under suitable conditions on $L$ (like an elliptic coercive (pseudo) differential operator of high enough order) the operator $K$ turns out to be the reproducing kernel of a Hilbert space of vector fields which is contained in the space of either $C_{b}^{1}$ (bounded $C^{1}$ with 
respect to $\bar{g}$ ) or $C_{b}^{2}$ vector fields. See [108, Chapter 12], 68, and [80 for uses of the reproducing Hilbert space approach. The right invariant metric is then defined as in 8.5 , where $\langle,\rangle_{\mathfrak{X}_{\mathcal{A}}(M)}$ is the duality:

$$
G_{\varphi}^{L}(X \circ \varphi, Y \circ \varphi)=G_{\mathrm{Id}}^{L}(X, Y)=\gamma(X, Y)=\langle L(X), Y\rangle_{\mathfrak{X}_{\mathcal{A}}(M)} .
$$

For example, the Sobolev metric of order $s$ corresponds to the inertia operator $L(X)=\left(1+\Delta^{\bar{g}}\right)^{s}(X) \cdot \operatorname{vol}(\bar{g})$. Examples of metrics are:

$$
\begin{aligned}
G_{\mathrm{Id}}^{0}(X, Y) & =\int_{M} \bar{g}(X, Y) \operatorname{vol}(\bar{g}) & & \text { the } L^{2} \text { metric, } \\
G_{\mathrm{Id}}^{s}(X, Y) & =\int_{M} \bar{g}\left(\left(1+\Delta^{\bar{g}}\right)^{s} X, Y\right) \operatorname{vol}(\bar{g}) & & \text { a Sobolev metric of order } s, \\
G_{\mathrm{Id}}^{\dot{H}^{1}}(X, Y) & =\int_{\mathbb{R}} X^{\prime} \cdot Y^{\prime} d x=-\int_{\mathbb{R}} X^{\prime \prime} Y d x & & \text { where } X, Y \in \mathfrak{X}_{\mathcal{A}}(\mathbb{R}) .
\end{aligned}
$$

As explained in 8.8 the geodesic equation on $\operatorname{Diff}_{\mathcal{A}}(M)$ is given as follows: Let $\varphi:[a, b] \rightarrow \operatorname{Diff}_{\mathcal{A}}(M)$ be a smooth curve. In terms of its right logarithmic derivative

$$
u:[a, b] \rightarrow \mathfrak{X}_{\mathcal{A}}(M), \quad u(t):=\varphi^{*} \kappa\left(\partial_{t}\right)=\varphi^{\prime}(t) \circ \varphi(t)^{-1},
$$

the geodesic equation is

$$
L\left(u_{t}\right)=L\left(\partial_{t} u\right)=-\operatorname{ad}(u)^{*} L(u) .
$$

The condition for the existence of the geodesic equation is as follows:

$$
X \mapsto K\left(\operatorname{ad}(X)^{*} L(X)\right)
$$

is bounded quadratic $\mathfrak{X}_{\mathcal{A}}(M) \rightarrow \mathfrak{X}_{\mathcal{A}}(M)$. Using Lie derivatives, the computation of $\operatorname{ad}_{X}^{*}$ is especially simple. Namely, for any section $\omega$ of $T^{*} M \otimes \mathrm{vol}$ and vector fields $\xi, \eta \in \mathfrak{X}_{\mathcal{A}}(M)$, we have:

$$
\int_{M}(\omega,[\xi, \eta])=\int_{M}\left(\omega, \mathcal{L}_{\xi}(\eta)\right)=-\int_{M}\left(\mathcal{L}_{\xi}(\omega), \eta\right),
$$

hence $\operatorname{ad}_{\xi}^{*}(\omega)=+\mathcal{L}_{\xi}(\omega)$. Thus the Hamiltonian version of the geodesic equation on the smooth dual $L\left(\mathfrak{X}_{\mathcal{A}}(M)\right) \subset \Gamma_{C_{b}^{2}}\left(T^{*} M \otimes \operatorname{vol}\right)$ becomes

$$
\partial_{t} \alpha=-\operatorname{ad}_{K(\alpha)}^{*} \alpha=-\mathcal{L}_{K(\alpha)} \alpha,
$$

or, keeping track of everything,

$$
\partial_{t} \varphi=u \circ \varphi, \quad \partial_{t} \alpha=-\mathcal{L}_{u} \alpha \quad u=K(\alpha)=\alpha^{\sharp}, \quad \alpha=L(u)=u^{b} .
$$

8.9. Theorem. Geodesic distance vanishes on $\operatorname{Diff}_{\mathcal{A}}(M)$ for any Sobolev metric of order $s<\frac{1}{2}$. If $M=S^{1} \times C$ with $C$ compact, then geodesic distance vanishes also for $s=\frac{1}{2}$. It also vanishes for the $L^{2}$-metric on the Virasoro group $\mathbb{R} \rtimes \operatorname{Diff}_{\mathcal{A}}(\mathbb{R})$.

Geodesic distance is positive on $\operatorname{Diff}_{\mathcal{A}}(M)$ for any Sobolev metric of order $s \geq 1$. If $\operatorname{dim}(M)=1$ then geodesic distance is also positive for $s>\frac{1}{2}$. 
This is proved in [8, 14, and 6]. Note that low order Sobolev metrics have geodesic equations corresponding to well-known nonlinear PDEs: On $\operatorname{Diff}\left(S^{1}\right)$ or Diff $\mathcal{A}_{\mathcal{A}}(\mathbb{R})$ the $L^{2}$-geodesic equation is Burgers' equation, on the Virasoro group it is the $\mathrm{KdV}$ equation, and the (standard) $H^{1}$-geodesic is (in both cases a variant of) the Camassa-Holm equation; see [10, 7.2] for a more comprehensive overview. All these are completely integrable infinite dimensional Hamiltonian systems.

8.10. Theorem. Let $(M, \bar{g})$ be a compact Riemannian manifold. Then the geodesic equation is locally well-posed on $\operatorname{Diff}_{\mathcal{A}}(M)$ and the geodesic exponential mapping is a local diffeomorphism for a Sobolev metric of integer order $s \geq 1$. For a Sobolev metric of integer order $s>\frac{\operatorname{dim}(M)+3}{2}$ the geodesic equation is even globally well-posed, so that $\left(\operatorname{Diff}_{\mathcal{A}}(M), G^{s}\right)$ is geodesically complete. This is also true for non-integer order $s$ if $M=\mathbb{R}^{n}$.

For $M=S^{1}$, the geodesic equation is locally well-posed even for $s \geq \frac{1}{2}$.

For these results see [12], 33], 32], [11].

8.11. Theorem. 9] For $\mathcal{A} \in\left\{C_{c}^{\infty}, \mathcal{S}, W^{\infty, 1}\right\}$ let

$$
\mathcal{A}_{1}(\mathbb{R})=\left\{f \in C^{\infty}(R): f^{\prime} \in \mathcal{A}(\mathbb{R}), f(-\infty)=0\right\}
$$

and let $\operatorname{Diff}_{\mathcal{A}_{1}}(\mathbb{R})=\left\{\varphi=\operatorname{Id}+f: f \in \mathcal{A}_{1}(\mathbb{R}), f^{\prime}>-1\right\}$. These are all regular Lie groups. The right invariant weak Riemannian metric

$$
G_{\text {Id }}^{\dot{H}^{1}}(X, Y)=\int_{\mathbb{R}} X^{\prime} Y^{\prime} d x
$$

is positive definite both on $\operatorname{Diff}_{\mathcal{A}}(\mathbb{R})$ where it does not admit a geodesic equation (a non-robust weak Riemannian manifold), and on Diff $\mathcal{A}_{1}(\mathbb{R})$ where it admits a geodesic equation but not in the stronger sense of Arnold. On $\operatorname{Diff}_{\mathcal{A}_{1}}(\mathbb{R})$ the geodesic equation is the Hunter-Saxton equation

$$
\left(\varphi_{t}\right) \circ \varphi^{-1}=u, \quad u_{t}=-u u_{x}+\frac{1}{2} \int_{-\infty}^{x}\left(u_{x}(z)\right)^{2} d z
$$

and the induced geodesic distance is positive. We define the R-map by:

$$
R: \operatorname{Diff}_{\mathcal{A}_{1}}(\mathbb{R}) \rightarrow \mathcal{A}\left(\mathbb{R}, \mathbb{R}_{>-2}\right) \subset \mathcal{A}(\mathbb{R}, \mathbb{R}), \quad R(\varphi)=2\left(\left(\varphi^{\prime}\right)^{1 / 2}-1\right) .
$$

The $R$-map is invertible with inverse

$$
R^{-1}: \mathcal{A}\left(\mathbb{R}, \mathbb{R}_{>-2}\right) \rightarrow \operatorname{Diff}_{\mathcal{A}_{1}}(\mathbb{R}), \quad R^{-1}(\gamma)(x)=x+\frac{1}{4} \int_{-\infty}^{x} \gamma^{2}+4 \gamma d x
$$

The pullback of the flat $L^{2}$-metric via $R$ is the $\dot{H}^{1}$-metric on $\operatorname{Diff}_{\mathcal{A}}(\mathbb{R})$, i.e., $R^{*}\langle\cdot, \cdot\rangle_{L^{2}(d x)}=G^{\dot{H}^{1}}$. Thus the space $\left(\operatorname{Diff}_{\mathcal{A}_{1}}(\mathbb{R}), \dot{H}^{1}\right)$ is a flat space in the sense of Riemannian geometry. There are explicit formulas for geodesics, geodesic distance, and geodesic splines, even for more restrictive spaces $\mathcal{A}_{1}$ like Denjoy-Carleman ultradifferentiable function classes. There are also solitonlike solutions. (Diff $\left.\mathcal{A}_{1}(\mathbb{R}), G^{\dot{H}^{1}}\right)$ is geodesically convex, but not geodesically complete; the geodesic completion is the smooth semigroup

$$
\operatorname{Mon}_{\mathcal{A}_{1}}=\left\{\varphi=\operatorname{Id}+f: f \in \mathcal{A}_{1}(\mathbb{R}), f^{\prime} \geq-1\right\} .
$$


Any geodesic can hit the subgroup $\operatorname{Diff}_{\mathcal{A}}(\mathbb{R}) \subset \operatorname{Diff}_{\mathcal{A}_{1}}(\mathbb{R})$ at most twice.

8.12. Trouvé groups for reproducing kernel Hilbert spaces. This is the origin of the notion of a Trouvé group. It puts the approach of 8.1 to 8.11 upside down and gets rid of the use of the Lie algebra structure on the space of vector fields. If the generating space $\mathcal{A}$ of vector fields on $\mathbb{R}^{d}$ for the Trouvé group $\mathcal{G}_{\mathcal{A}}$ (see 6.12 is a reproducing kernel Hilbert space $\left(\mathcal{A}\left(\mathbb{R}^{d}, \mathbb{R}^{d}\right),\langle,,\rangle_{\mathcal{A}}\right)$ contained in $C_{b}^{1}$, then

$$
\operatorname{dist}(\mathrm{Id}, \varphi):=\inf \left\{\int_{0}^{1}\|X(t)\|_{\mathcal{A}} d t: X \in \mathcal{F}_{\mathcal{A}}, \text { evol }^{X}=\varphi\right\}
$$

defines a metric which makes the Trouvé group $\mathcal{G}_{\mathcal{A}}$ into a topological group; see [103, [108. This is widely used for the Large Deformation Diffeomorphic Metric Matching (LDDMM) method in image analysis and computational anatomy. The most popular reproducing kernel Hilbert space is the one where the kernel is a Gaussian $e^{-|x|^{2} / \sigma}$. Here the space $\mathcal{A}$ is a certain space of entire real analytic functions, and a direct description of the Trouvé group is severely lacking.

\section{Robust weak Riemannian manifolds and Riemannian SUBMERSIONS}

9.1. Robust weak Riemannian manifolds. Some constructions may lead to vector fields whose values do not lie in $T_{x} M$, but in the Hilbert space completion $\overline{T_{x} M}$ with respect to the weak inner product $g_{x}$. We need that $\bigcup_{x \in M} \overline{T_{x} M}$ forms a smooth vector bundle over $M$. In a coordinate chart on open $U \subset M,\left.T M\right|_{U}$ is a trivial bundle $U \times V$ and all the inner products $g_{x}, x \in U$ define inner products on the same topological vector space $V$. They all should be bounded with respect to each other, so that the completion $\bar{V}$ of $V$ with respect to $g_{x}$ does not depend on $x$ and $\bigcup_{x \in U} \overline{T_{x} M} \cong U \times \bar{V}$. This means that $\bigcup_{x \in M} \overline{T_{x} M}$ forms a smooth vector bundle over $M$ with trivializations the linear extensions of the trivializations of the tangent bundle $T M \rightarrow M$. Chart changes should respect this. This is a compatibility property between the weak Riemannian metric and some smooth atlas of $M$.

Definition A convenient weak Riemannian manifold $(M, g)$ will be called a robust Riemannian manifold if

- The Levi-Civita convariant derivative of the metric $g$ exists: The symmetric gradients should exist and be smooth.

- The completions $\overline{T_{x} M}$ form a smooth vector bundle as above.

9.2. Theorem. If a right invariant weak Riemannian metric on a regular Lie group admits the Levi-Civita covariant derivative, then it is already robust.

Proof. By right invariance, each right translation $T \mu^{g}$ extends to an isometric isomorphisms $\overline{T_{x} G} \rightarrow \overline{T_{x g} G}$. By the smooth uniform boundedness theorem these isomorphisms depend smoothly on $g \in G$. 
9.3. Covariant curvature and O'Neill's formula. In [68, 2.2] one finds the following formula for the numerator of sectional curvature, which is valid for closed smooth 1-forms $\alpha, \beta \in \Omega_{g}^{1}(M)$ on a weak Riemannian manifold $(M, g)$. Recall that we view $g: T M \rightarrow T^{*} M$ and so $g^{-1}$ is the dual inner product on $g(T M)$ and $\alpha^{\sharp}=g^{-1}(\alpha)$.

$$
\begin{aligned}
& g\left(R\left(\alpha^{\sharp}, \beta^{\sharp}\right) \alpha^{\sharp}, \beta^{\sharp}\right)= \\
& -\frac{1}{2} \alpha^{\sharp} \alpha^{\sharp}\left(\|\beta\|_{g^{-1}}^{2}\right)-\frac{1}{2} \beta^{\sharp} \beta^{\sharp}\left(\|\alpha\|_{g^{-1}}^{2}\right)+\frac{1}{2}\left(\alpha^{\sharp} \beta^{\sharp}+\beta^{\sharp} \alpha^{\sharp}\right) g^{-1}(\alpha, \beta) \\
& \left.\quad\left(\text { last line }=-\alpha^{\sharp} \beta\left(\left[\alpha^{\sharp}, \beta^{\sharp}\right]\right)+\beta^{\sharp} \alpha\left(\left[\alpha^{\sharp}, \beta^{\sharp}\right]\right]\right)\right) \\
& -\frac{1}{4}\left\|d\left(g^{-1}(\alpha, \beta)\right)\right\|_{g^{-1}}^{2}+\frac{1}{4} g^{-1}\left(d\left(\|\alpha\|_{g^{-1}}^{2}\right), d\left(\|\beta\|_{g^{-1}}^{2}\right)\right) \\
& +\frac{3}{4}\left\|\left[\alpha^{\sharp}, \beta^{\sharp}\right]\right\|_{g}^{2}
\end{aligned}
$$

This is called Mario's formula since Mario Micheli derived the coordinate version in his 2008 thesis. Each term depends only on $g^{-1}$ with the exception of the last term. The role of the last term (which we call the O'Neill term) will become clear in the next result. Let $p:\left(E, g_{E}\right) \rightarrow\left(B, g_{B}\right)$ be a Riemannian submersion between infinite dimensional robust Riemannian manifolds; i.e., for each $b \in B$ and $x \in E_{b}:=p^{-1}(b)$ the tangent mapping $T_{x} p:\left(T_{x} E, g_{E}\right) \rightarrow$ $\left(T_{b} B, g_{B}\right)$ is a surjective metric quotient map so that

$$
\left\|\xi_{b}\right\|_{g_{B}}:=\inf \left\{\left\|X_{x}\right\|_{g_{E}}: X_{x} \in T_{x} E, T_{x} p \cdot X_{x}=\xi_{b}\right\} .
$$

The infinimum need not be attained in $T_{x} E$ but will be in the completion $\overline{T_{x} E}$. The orthogonal subspace $\left\{Y_{x}: g_{E}\left(Y_{x}, T_{x}\left(E_{b}\right)\right)=0\right\}$ will therefore be taken in $\overline{T_{x}\left(E_{b}\right)}$ in $T_{x} E$. If $\alpha_{b}=g_{B}\left(\alpha_{b}^{\sharp}, \quad\right) \in g_{B}\left(T_{b} B\right) \subset T_{b}^{*} B$ is an element in the $g_{B}$-smooth dual, then $p^{*} \alpha_{b}:=\left(T_{x} p\right)^{*}\left(\alpha_{b}\right)=g_{B}\left(\alpha_{b}^{\sharp}, T_{x} p \quad\right): T_{x} E \rightarrow \mathbb{R}$ is in $T_{x}^{*} E$ but in general it is not an element in the smooth dual $g_{E}\left(T_{x} E\right)$. It is, however, an element of the Hilbert space completion $\overline{g_{E}\left(T_{x} E\right)}$ of the $g_{E}$-smooth dual $g_{E}\left(T_{x} E\right)$ with respect to the norm \|\|$_{g_{E}^{-1}}$, and the element $g_{E}^{-1}\left(p^{*} \alpha_{b}\right)=:\left(p^{*} \alpha_{b}\right)^{\sharp}$ is in the \|\|$_{g_{E}}$-completion $\overline{T_{x} E}$ of $T_{x} E$. We can call $g_{E}^{-1}\left(p^{*} \alpha_{b}\right)=:\left(p^{*} \alpha_{b}\right)^{\sharp}$ the horizontal lift of $\alpha_{b}^{\sharp}=g_{B}^{-1}\left(\alpha_{b}\right) \in T_{b} B$.

9.4. Theorem. 68, 2.6] Let $p:\left(E, g_{E}\right) \rightarrow\left(B, g_{B}\right)$ be a Riemannian submersion between infinite dimensional robust Riemannian manifolds. Then for closed 1-forms $\alpha, \beta \in \Omega_{g_{B}}^{1}(B)$ O'Neill's formula holds in the form:

$$
\begin{aligned}
g_{B}\left(R^{B}\left(\alpha^{\sharp}, \beta^{\sharp}\right) \beta^{\sharp}, \alpha^{\sharp}\right)= & g_{E}\left(R^{E}\left(\left(p^{*} \alpha\right)^{\sharp},\left(p^{*} \beta\right)^{\sharp}\right)\left(p^{*} \beta\right)^{\sharp},\left(p^{*} \alpha\right)^{\sharp}\right) \\
& +\frac{3}{4}\left\|\left[\left(p^{*} \alpha\right)^{\sharp},\left(p^{*} \beta\right)^{\sharp}\right]^{\text {ver }}\right\|_{g_{E}}^{2}
\end{aligned}
$$

Proof. The last (O'Neill) term is the difference between curvature on $E$ and the pullback of the curvature on $B$.

9.5. Semilocal version of Mario's formula, force, and stress. In all interesting examples of orbits of diffeomorphisms groups through a template shape, Mario's covariant curvature formula leads to complicated and impenetrable formulas. Efforts to break this down to comprehensible pieces led to 
the concepts of symmetrized force and (shape-) stress explained below. Since acceleration sits in the second tangent bundle, one either needs a covariant derivative to map it down to the tangent bundle, or at least rudiments of local charts. In 68] we managed the local version. Interpretations in mechanics or elasticity theory are still lacking.

Let $(M, g)$ be a robust Riemannian manifold, $x \in M, \alpha, \beta \in g_{x}\left(T_{x} M\right)$. Assume we are given local smooth vector fields $X_{\alpha}$ and $X_{\beta}$ such that:

(1) $X_{\alpha}(x)=\alpha^{\sharp}(x), \quad X_{\beta}(x)=\beta^{\sharp}(x)$,

(2) Then $\alpha^{\sharp}-X_{\alpha}$ is zero at $x$. Therefore it has a well-defined derivative $D_{x}\left(\alpha^{\sharp}-X_{\alpha}\right)$ lying in $\operatorname{Hom}\left(T_{x} M, T_{x} M\right)$. For a vector field $Y$ we have $D_{x}\left(\alpha^{\sharp}-X_{\alpha}\right) \cdot Y_{x}=\left[Y, \alpha^{\sharp}-X_{\alpha}\right](x)=\left.\mathcal{L}_{Y}\left(\alpha^{\sharp}-X_{\alpha}\right)\right|_{x}$. The same holds for $\beta$.

(3) $\mathcal{L}_{X_{\alpha}}(\alpha)=\mathcal{L}_{X_{\alpha}}(\beta)=\mathcal{L}_{X_{\beta}}(\alpha)=\mathcal{L}_{X_{\beta}}(\beta)=0$,

(4) $\left[X_{\alpha}, X_{\beta}\right]=0$.

Locally constant 1 -forms and vector fields will do. We then define:

$$
\begin{aligned}
\mathcal{F}(\alpha, \beta): & =\frac{1}{2} d\left(g^{-1}(\alpha, \beta)\right), \quad \text { a } 1 \text {-form on } M \text { called the force, } \\
\mathcal{D}(\alpha, \beta)(x): & =D_{x}\left(\beta^{\sharp}-X_{\beta}\right) \cdot \alpha^{\sharp}(x) \\
& =d\left(\beta^{\sharp}-X_{\beta}\right) \cdot \alpha^{\sharp}(x), \quad \in T_{x} M \text { called the stress. } \\
\Longrightarrow & \mathcal{D}(\alpha, \beta)(x)-\mathcal{D}(\beta, \alpha)(x)=\left[\alpha^{\sharp}, \beta^{\sharp}\right](x)
\end{aligned}
$$

Then in terms of force and stress the numerator of sectional curvature looks as follows:

$$
\begin{aligned}
& g\left(R\left(\alpha^{\sharp}, \beta^{\sharp}\right) \beta^{\sharp}, \alpha^{\sharp}\right)(x)=R_{11}+R_{12}+R_{2}+R_{3}, \quad \text { where } \\
& R_{11}=\frac{1}{2}\left(\mathcal{L}_{X_{\alpha}}^{2}\left(g^{-1}\right)(\beta, \beta)-2 \mathcal{L}_{X_{\alpha}} \mathcal{L}_{X_{\beta}}\left(g^{-1}\right)(\alpha, \beta)+\mathcal{L}_{X_{\beta}}^{2}\left(g^{-1}\right)(\alpha, \alpha)\right)(x), \\
& R_{12}=\langle\mathcal{F}(\alpha, \alpha), \mathcal{D}(\beta, \beta)\rangle+\langle\mathcal{F}(\beta, \beta), \mathcal{D}(\alpha, \alpha)\rangle-\langle\mathcal{F}(\alpha, \beta), \mathcal{D}(\alpha, \beta)+\mathcal{D}(\beta, \alpha)\rangle \\
& \left.R_{2}=\left(\|\mathcal{F}(\alpha, \beta)\|_{g^{-1}}^{2}-\langle\mathcal{F}(\alpha, \alpha)), \mathcal{F}(\beta, \beta)\right\rangle_{g^{-1}}\right)(x), \\
& R_{3}=-\frac{3}{4}\|\mathcal{D}(\alpha, \beta)-\mathcal{D}(\beta, \alpha)\|_{g_{x}}^{2} .
\end{aligned}
$$

9.6. Landmark space as homogeneous space of solitons. This subsection is based on 67]; the method explained here has many applications in computational anatomy and elsewhere, under the name LDDMM (large diffeomorphic deformation metric matching).

A landmark $q=\left(q_{1}, \ldots, q_{N}\right)$ is an $N$-tuple of distinct points in $\mathbb{R}^{n}$; landmark space Land $^{N}\left(\mathbb{R}^{n}\right) \subset\left(\mathbb{R}^{n}\right)^{N}$ is open. Let $q^{0}=\left(q_{1}^{0}, \ldots, q_{N}^{0}\right)$ be a fixed standard template landmark. Then we have the surjective mapping

$$
\begin{aligned}
& \operatorname{ev}_{q^{0}}: \operatorname{Diff}_{\mathcal{A}}\left(\mathbb{R}^{n}\right) \rightarrow \operatorname{Land}^{N}\left(\mathbb{R}^{n}\right), \\
& \varphi \mapsto \operatorname{ev}_{q^{0}}(\varphi)=\varphi\left(q^{0}\right)=\left(\varphi\left(q_{1}^{0}\right), \ldots, \varphi\left(q_{N}^{0}\right)\right) .
\end{aligned}
$$

Given a Sobolev metric of order $s>\frac{n}{2}+2$ on $\operatorname{Diff}_{\mathcal{A}}\left(\mathbb{R}^{n}\right)$, we want to induce a Riemannian metric on Land ${ }^{N}\left(\mathbb{R}^{n}\right)$ such that $\mathrm{ev}_{q_{0}}$ becomes a Riemannian submersion. 
The fiber of $\operatorname{ev}_{q^{0}}$ over a landmark $q=\varphi_{0}\left(q^{0}\right)$ is

$$
\begin{aligned}
\left\{\varphi \in \operatorname{Diff}_{\mathcal{A}}\left(\mathbb{R}^{n}\right): \varphi\left(q^{0}\right)=q\right\} & =\varphi_{0} \circ\left\{\varphi \in \operatorname{Diff}_{\mathcal{A}}\left(\mathbb{R}^{n}\right): \varphi\left(q^{0}\right)=q^{0}\right\} \\
& =\left\{\varphi \in \operatorname{Diff}_{\mathcal{A}}\left(\mathbb{R}^{n}\right): \varphi(q)=q\right\} \circ \varphi_{0} .
\end{aligned}
$$

The tangent space to the fiber is

$$
\left\{X \circ \varphi_{0}: X \in \mathfrak{X}_{\mathcal{S}}\left(\mathbb{R}^{n}\right), X\left(q_{i}\right)=0 \text { for all } i\right\} .
$$

A tangent vector $Y \circ \varphi_{0} \in T_{\varphi_{0}} \operatorname{Diff}_{\mathcal{S}}\left(\mathbb{R}^{n}\right)$ is $G_{\varphi_{0}}^{L}$-perpendicular to the fiber over $q$ if and only if

$$
\int_{\mathbb{R}^{n}}\langle L Y, X\rangle d x=0 \quad \forall X \text { with } X(q)=0 .
$$

If we require $Y$ to be smooth then $Y=0$. So we assume that $L Y=\sum_{i} P_{i} . \delta_{q_{i}}$, a distributional vector field with support in $q$. Here $P_{i} \in T_{q_{i}} \mathbb{R}^{n}$. But then

$$
\begin{gathered}
Y(x)=L^{-1}\left(\sum_{i} P_{i} \cdot \delta_{q_{i}}\right)=\int_{\mathbb{R}^{n}} K(x-y) \sum_{i} P_{i} \cdot \delta_{q_{i}}(y) d y=\sum_{i} K\left(x-q_{i}\right) \cdot P_{i} \\
T_{\varphi_{0}}\left(\operatorname{ev}_{q^{0}}\right) .\left(Y \circ \varphi_{0}\right)=Y\left(q_{k}\right)_{k}=\sum_{i}\left(K\left(q_{k}-q_{i}\right) \cdot P_{i}\right)_{k} .
\end{gathered}
$$

Now let us consider a tangent vector $P=\left(P_{k}\right) \in T_{q} \operatorname{Land}^{N}\left(\mathbb{R}^{n}\right)$. Its horizontal lift with footpoint $\varphi_{0}$ is $P^{\text {hor }} \circ \varphi_{0}$ where the vector field $P^{\text {hor }}$ on $\mathbb{R}^{n}$ is given as follows: Let $K^{-1}(q)_{k i}$ be the inverse of the $(N \times N)$-matrix $K(q)_{i j}=K\left(q_{i}-q_{j}\right)$. Then

$$
\begin{aligned}
P^{\text {hor }}(x) & =\sum_{i, j} K\left(x-q_{i}\right) K^{-1}(q)_{i j} P_{j}, \\
L\left(P^{\text {hor }}(x)\right) & =\sum_{i, j} \delta\left(x-q_{i}\right) K^{-1}(q)_{i j} P_{j} .
\end{aligned}
$$

Note that $P^{\text {hor }}$ is a vector field of class $H^{2 l-1}$.

The Riemannian metric on the finite dimensional manifold Land $^{N}$ induced by the $g^{L}$-metric on Diff $\mathcal{S}\left(\mathbb{R}^{n}\right)$ is given by

$$
\begin{aligned}
g_{q}^{L}(P, Q) & =G_{\varphi_{0}}^{L}\left(P^{\text {hor }}, Q^{\text {hor }}\right)=\int_{\mathbb{R}^{n}}\left\langle L\left(P^{\text {hor }}\right), Q^{\text {hor }}\right\rangle d x \\
& =\int_{\mathbb{R}^{n}}\left\langle\sum_{i, j} \delta\left(x-q_{i}\right) K^{-1}(q)_{i j} P_{j}, \sum_{k, l} K\left(x-q_{k}\right) K^{-1}(q)_{k l} Q_{l}\right\rangle d x \\
& =\sum_{i, j, k, l} K^{-1}(q)_{i j} K\left(q_{i}-q_{k}\right) K^{-1}(q)_{k l}\left\langle P_{j}, Q_{l}\right\rangle \\
g_{q}^{L}(P, Q) & =\sum_{k, l} K^{-1}(q)_{k l}\left\langle P_{k}, Q_{l}\right\rangle .
\end{aligned}
$$

The geodesic equation in vector form is:

$$
\ddot{q}_{n}=-\frac{1}{2} \sum_{k, i, j, l} K^{-1}(q)_{k i} \operatorname{grad} K\left(q_{i}-q_{j}\right)\left(K(q)_{i n}-K(q)_{j n}\right) K^{-1}(q)_{j l}\left\langle\dot{q}_{k}, \dot{q}_{l}\right\rangle
$$




$$
+\sum_{k, i} K^{-1}(q)_{k i}\left\langle\operatorname{grad} K\left(q_{i}-q_{n}\right), \dot{q}_{i}-\dot{q}_{n}\right\rangle \dot{q}_{k}
$$

The cotangent bundle $T^{*} \operatorname{Land}^{N}\left(\mathbb{R}^{n}\right)=\operatorname{Land}^{N}\left(\mathbb{R}^{n}\right) \times\left(\left(\mathbb{R}^{n}\right)^{N}\right)^{*} \ni(q, \alpha)$. We treat $\mathbb{R}^{n}$ like scalars; $\langle, \quad\rangle$ is always the standard inner product on $\mathbb{R}^{n}$. The inverse metric is then given by

$$
\left(g^{L}\right)_{q}^{-1}(\alpha, \beta)=\sum_{i, j} K(q)_{i j}\left\langle\alpha_{i}, \beta_{j}\right\rangle, \quad K(q)_{i j}=K\left(q_{i}-q_{j}\right) .
$$

The energy function is

$$
E(q, \alpha)=\frac{1}{2}\left(g^{L}\right)_{q}^{-1}(\alpha, \alpha)=\frac{1}{2} \sum_{i, j} K(q)_{i j}\left\langle\alpha_{i}, \alpha_{j}\right\rangle
$$

and its Hamiltonian vector field (using $\mathbb{R}^{n}$-valued derivatives to save notation) is

$$
H_{E}(q, \alpha)=\sum_{i, k=1}^{N}\left(K\left(q_{k}-q_{i}\right) \alpha_{i} \frac{\partial}{\partial q_{k}}+\operatorname{grad} K\left(q_{i}-q_{k}\right)\left\langle\alpha_{i}, \alpha_{k}\right\rangle \frac{\partial}{\partial \alpha_{k}}\right) .
$$

So the Hamiltonian version of the geodesic equation is the flow of this vector field:

$$
\left\{\begin{array}{l}
\dot{q}_{k}=\sum_{i} K\left(q_{i}-q_{k}\right) \alpha_{i} \\
\dot{\alpha}_{k}=-\sum_{i} \operatorname{grad} K\left(q_{i}-q_{k}\right)\left\langle\alpha_{i}, \alpha_{k}\right\rangle
\end{array}\right.
$$

We shall use stress and force to express the geodesic equation and curvature:

$$
\begin{aligned}
& \alpha_{k}^{\sharp}=\sum_{i} K\left(q_{k}-q_{i}\right) \alpha_{i}, \quad \alpha^{\sharp}=\sum_{i, k} K\left(q_{k}-q_{i}\right)\left\langle\alpha_{i}, \frac{\partial}{\partial q^{k}}\right\rangle \\
& \mathcal{D}(\alpha, \beta):=\sum_{i, j} d K\left(q_{i}-q_{j}\right)\left(\alpha_{i}^{\sharp}-\alpha_{j}^{\sharp}\right)\left\langle\beta_{j}, \frac{\partial}{\partial q_{i}}\right\rangle, \quad \text { the stress. } \\
& \mathcal{D}(\alpha, \beta)-\mathcal{D}(\beta, \alpha)=\left(D_{\alpha^{\sharp}} \beta^{\sharp}\right)-D_{\beta^{\sharp}} \alpha^{\sharp}=\left[\alpha^{\sharp}, \beta^{\sharp}\right], \quad \text { Lie bracket. } \\
& \mathcal{F}_{i}(\alpha, \beta)=\frac{1}{2} \sum_{k} \operatorname{grad} K\left(q_{i}-q_{k}\right)\left(\left\langle\alpha_{i}, \beta_{k}\right\rangle+\left\langle\beta_{i}, \alpha_{k}\right\rangle\right) \\
& \mathcal{F}(\alpha, \beta):=\sum_{i}\left\langle\mathcal{F}_{i}(\alpha, \beta), d q_{i}\right\rangle=\frac{1}{2} d g^{-1}(\alpha, \beta) \quad \text { the force. }
\end{aligned}
$$

The geodesic equation on $T^{*} \operatorname{Land}^{N}\left(\mathbb{R}^{n}\right)$ then becomes

$$
\left\{\begin{array}{l}
\dot{q}=\alpha^{\sharp} \\
\dot{\alpha}=-\mathcal{F}(\alpha, \alpha) .
\end{array}\right.
$$

Next we shall compute curvature via the cotangent bundle. From the semilocal version of Mario's formula for the numerator of the sectional curvature for constant 1-forms $\alpha, \beta$ on landmark space, where $\alpha_{k}^{\sharp}=\sum_{i} K\left(q_{k}-q_{i}\right) \alpha_{i}$, we get directly:

$$
g^{L}\left(R\left(\alpha^{\sharp}, \beta^{\sharp}\right) \alpha^{\sharp}, \beta^{\sharp}\right)=
$$




$$
\begin{gathered}
=\langle\mathcal{D}(\alpha, \beta)+\mathcal{D}(\beta, \alpha), \mathcal{F}(\alpha, \beta)\rangle \\
-\langle\mathcal{D}(\alpha, \alpha), \mathcal{F}(\beta, \beta)\rangle-\langle\mathcal{D}(\beta, \beta), \mathcal{F}(\alpha, \alpha)\rangle \\
-\frac{1}{2} \sum_{i, j}\left(d^{2} K\left(q_{i}-q_{j}\right)\left(\beta_{i}^{\sharp}-\beta_{j}^{\sharp}, \beta_{i}^{\sharp}-\beta_{j}^{\sharp}\right)\left\langle\alpha_{i}, \alpha_{j}\right\rangle\right. \\
\quad-2 d^{2} K\left(q_{i}-q_{j}\right)\left(\beta_{i}^{\sharp}-\beta_{j}^{\sharp}, \alpha_{i}^{\sharp}-\alpha_{j}^{\sharp}\right)\left\langle\beta_{i}, \alpha_{j}\right\rangle \\
\left.\quad+d^{2} K\left(q_{i}-q_{j}\right)\left(\alpha_{i}^{\sharp}-\alpha_{j}^{\sharp}, \alpha_{i}^{\sharp}-\alpha_{j}^{\sharp}\right)\left\langle\beta_{i}, \beta_{j}\right\rangle\right) \\
-\|\mathcal{F}(\alpha, \beta)\|_{g^{-1}}^{2}+g^{-1}(\mathcal{F}(\alpha, \alpha), \mathcal{F}(\beta, \beta)) . \\
+\frac{3}{4}\left\|\left[\alpha^{\sharp}, \beta^{\sharp}\right]\right\|_{g}^{2}
\end{gathered}
$$

9.7. Shape spaces of submanifolds as homogeneous spaces for the diffeomorphism group. Let $M$ be a compact manifold and $(N, \bar{g})$ a Riemannian manifold of bounded geometry as in subsection 3.6. The diffeomorphism group $\operatorname{Diff}_{\mathcal{A}}(N)$ acts also from the left on the manifold of $\operatorname{Emb}(M, N)$ embeddings and also on the nonlinear Grassmannian or differentiable Chow variety $B(M, N)=\operatorname{Emb}(M, N) / \operatorname{Diff}(M)$. For a Sobolev metric of order $s>\frac{\operatorname{dim}(N)}{2}+2$ one can then again induce a Riemannian metric on each $\operatorname{Diff}_{\mathcal{A}}(N)$-orbit, as we did above for landmark spaces. This is done in 68, where the geodesic equation is computed and where curvature is described in terms of stress and force. 


\section{REFERENCES}

[1] R. Abraham. Lectures of Smale on differential topology. Columbia University, New York, 1962. Lecture Notes. 3

[2] H. Alzaareer and A. Schmeding. Differentiable mappings on products with different degrees of differentiability in the two factors. Expo. Math., 33(2):184-222, 2015. 19

[3] L. F. A. Arbogast. Du calcul des dérivations. Levrault, Strasbourg, 1800. 22

[4] V. Arnold. Sur la géometrie differentielle des groupes de Lie de dimension infinie et ses applications à l'hydrodynamique des fluides parfaits. Ann. Inst. Fourier, 16:319361, 1966. 51,53

[5] B. Arslan, A. P. Goncharov, and M. Kocatepe. Spaces of Whitney functions on Cantor-type sets. Canad. J. Math., 54(2):225-238, 2002.20

[6] M. Bauer, M. Bruveris, P. Harms, and P. W. Michor. Vanishing geodesic distance for the Riemannian metric with geodesic equation the KdV-equation. Ann. Global Anal. Geom., 41(4):461-472, 2012.49 55

[7] M. Bauer, M. Bruveris, P. Harms, and P. W. Michor. Smooth perturbations of the functional calculus and applications to Riemannian geometry on spaces of metrics, 2018. arXiv:1810.03169. 20

[8] M. Bauer, M. Bruveris, and P. W. Michor. Geodesic distance for right invariant Sobolev metrics of fractional order on the diffeomorphism group ii. Ann. Global Anal. Geom., 44(4):361-368, 2013. 55

[9] M. Bauer, M. Bruveris, and P. W. Michor. The homogeneous Sobolev metric of order one on diffeomorphism groups on the real line. Journal of Nonlinear Science, 24(5):769-808, 2014. $51,52,55$

[10] M. Bauer, M. Bruveris, and P. W. Michor. Overview of the geometries of shape spaces and diffeomorphism groups. J. Math. Imaging Vis., 50:60-97, 2014. 355

[11] M. Bauer, J. Escher, and B. Kolev. Local and global well-posedness of the fractional order EPDiff equation on $\mathbb{R}^{d}$. Journal of Differential Equations, 2015. arXiv:1411.4081. 55

[12] M. Bauer, P. Harms, and P. W. Michor. Sobolev metrics on shape space of surfaces. J. Geom. Mech., 3(4):389-438, 2011. $48,49,55$

[13] M. Bauer, P. Harms, and P. W. Michor. Almost local metrics on shape space of hypersurfaces in $n$-space. SIAM J. Imaging Sci., 5(1):244-310, 2012. 49

[14] M. Bauer, P. Harms, and P. W. Michor. Sobolev metrics on the manifold of all Riemannian metrics. J. Differential Geom., 94(2):187-208, 2013. 55

[15] E. Bierstone. Extension of Whitney fields from subanalytic sets. Invent. Math., 46:277-300, 1978. 13

[16] J. Boman. Differentiability of a function and of its compositions with functions of one variable. Math. Scand., 20:249-268, 1967. 5

[17] R. Brown. Some problems of algebraic topology. PhD thesis, 1961. Oxford. 4

[18] R. Brown. Ten topologies for $X \times Y$. Quart. J. Math., 14:303-319, 1963. 4

[19] R. Brown. Function spaces and product topologies. Quart. J. Math., 15:238-250, 1964. 4

[20] M. Bruveris. Completeness properties of Sobolev metrics on the space of curves. $J$. Geom. Mech., 7(2):125-150, 2015. arXiv:1407.0601.49

[21] M. Bruveris, P. W. Michor, and D. Mumford. Geodesic completeness for Sobolev metrics on the space of immersed plane curves. Forum Math. Sigma, 2:e19 (38 pages), 2014. 49

[22] M. Bruveris and F.-X. Vialard. On completeness of groups of diffeomorphisms. J. Eur. Math. Soc., 19(5):1507-1544, 2017. 35

[23] V. Cervera, F. Mascaro, and P. W. Michor. The orbit structure of the action of the diffeomorphism group on the space of immersions. Diff. Geom. Appl., 1:391-401, 1991. 36 
[24] B. Conrad. Stokes' theorem with corners. Math 396 Handout, Stanford University. http://math.stanford.edu/ conrad/diffgeomPage/handouts/stokescorners.pdf 10

[25] A. Douady and L. Hérault. Arrondissement des variétés à coins. Comment. Math. Helv., 48:484-491, 1973. Appendice à A.Borel and J.-P. Serre: Corners and arithmetic groups. 8

[26] D. Ebin and J. Marsden. Groups of diffeomorphisms and the motion of an incompressible fluid. Ann. Math., 92:102-163, 1970. 3

[27] J. Eells. A setting for global analysis. Bull AMS, 72:571-807, 1966. 3

[28] J. Eells, Jr. On the geometry of function spaces. In Symposium internacional de topologia algebraica International symposium on algebraic topology, pages 303-308. Universidad Nacional Autónoma de México and UNESCO, Mexico City, 1958. 3

[29] J. Eichhorn. Gauge theory on open manifolds of bounded geometry. Internat. J. Modern Physics, 7:3927-3977, 1992.11.

[30] J. Eichhorn. Global analysis on open manifolds. Nova Science Publishers Inc., New York, 2007. 1153

[31] H. I. Eliasson. Geometry of manifolds of maps. J. Differential Geometry, 1:169-194, 1967. 3

[32] J. Escher and B. Kolev. Geodesic completeness for Sobolev $H^{s}$-metrics on the diffeomorphisms group of the circle. Journal of Evolution Equations, 2014. 55

[33] J. Escher and B. Kolev. Right-invariant Sobolev metrics of fractional order on the diffeomorphism group of the circle. J. Geom. Mech., 6:335-372, 2014.55

[34] C. F. Faà di Bruno. Note sur une nouvelle formule du calcul différentielle. Quart. J. Math., 1:359-360, 1855.22

[35] L. Fantappié. I functionali analitici. Atti Accad. Naz. Lincei, Mem. 3-11:453-683, 1930. 6

[36] L. Fantappié. Überblick über die Theorie der analytischen Funktionale und ihre Anwendungen. Jahresber. Deutsch. Mathem. Verein., 43:1-25, 1933. 6

[37] C.-A. Faure. Théorie de la différentiation dans les espaces convenables. $\mathrm{PhD}$ thesis, Université de Genéve, 1991.7

[38] C.-A. Faure and A. Frölicher. Hölder differentiable maps and their function spaces. In Categorical topology and its relation to analysis, algebra and combinatorics (Prague, 1988), pages 135-142. World Sci. Publ., Teaneck, NJ, 1989. 7

[39] C. Fefferman. $C^{m}$ extension by linear operators. Ann. of Math. (2), 166(3):779-835, 2007. 17

[40] L. Frerick. Extension operators for spaces of infinite differentiable Whitney jets. $J$. Reine Angew. Math., 602:123-154, 2007.13, 14,15,20

[41] L. Frerick, E. Jordá, and J. Wengenroth. Tame linear extension operators for smooth Whitney functions. J. Funct. Anal., 261(3):591-603, 2011.14 20

[42] L. Frerick, E. Jordá, and J. Wengenroth. Whitney extension operators without loss of derivatives. Rev. Mat. Iberoam., 32(2):377-390, 2016. 14

[43] L. Frerick and J. Wengenroth. Private communication. 2019. 15,20

[44] A. Frölicher and A. Kriegl. Linear spaces and differentiation theory. J. Wiley, Chichester, 1988. Pure and Applied Mathematics. 3, 6, 7

[45] F. Gay-Balmaz and C. Vizman. Principal bundles of embeddings and nonlinear Grassmannians. Ann. Global Anal. Geom., 46(3):293-312, 2014. 1135

[46] H. Glöckner. Measurable regularity properties of infinite-dimensional Lie groups, 2015. arXiv:1601.02568. 28

[47] R. E. Greene. Complete metrics of bounded curvature on noncompact manifolds. Arch. Math. (Basel), 31(1):89-95, 1978/79. 11

[48] J. Gutknecht. Die $C_{\Gamma}^{\infty}$-Struktur auf der Diffeomorphismengruppe einer kompakten Mannigfaltigkeit. 1977. Doctoral Thesis, ETH Zürich. 3

[49] M. Hanusch. Regularity of Lie groups, 2017. arXiv:1711.03508. 28

[50] K. Hart. Sets with positive Lebesgue measure boundary. MathOverflow question, 2010. https://mathoverflow.net/q/26000 20 
[51] P. Iglesias-Zemmour. Diffeology, volume 185 of Mathematical Surveys and Monographs. American Mathematical Society, Providence, RI, 2013. 8

[52] E. Klassen and P. W. Michor. Closed surfaces with different shapes that are indistinguishable by the SRNF. 2019. Preprint. 20

[53] Y. A. Kordyukov. $L^{p}$-theory of elliptic differential operators on manifolds of bounded geometry. Acta Appl. Math., 23(3):223-260, 1991. 11.

[54] A. Kriegl and P. W. Michor. A convenient setting for real analytic mappings. Acta Mathematica, 165:105-159, 1990. 6

[55] A. Kriegl and P. W. Michor. The Convenient Setting for Global Analysis. AMS,

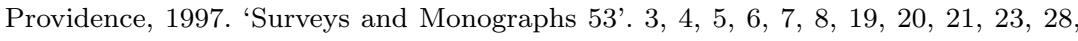
3248

[56] A. Kriegl and P. W. Michor. Regular infinite-dimensional Lie groups. J. Lie Theory, 7(1):61-99, 1997. 28

[57] A. Kriegl, P. W. Michor, and A. Rainer. The convenient setting for non-quasianalytic Denjoy-Carleman differentiable mappings. J. Funct. Anal., 256:3510-3544, 2009.6

[58] A. Kriegl, P. W. Michor, and A. Rainer. The convenient setting for quasianalytic Denjoy-Carleman differentiable mappings. J. Funct. Anal., 261:1799-1834, 2011.6

[59] A. Kriegl, P. W. Michor, and A. Rainer. The convenient setting for Denjoy-Carleman differentiable mappings of Beurling and Roumieu type. Revista Matematica Complutense, 2015. 6

[60] A. Kriegl, P. W. Michor, and A. Rainer. An exotic zoo of diffeomorphism groups on $\mathbb{R}^{n}$. Ann. Glob. Anal. Geom., 47(2):179-222, 2015.7] 31, 32,34

[61] A. Kriegl, P. W. Michor, and A. Rainer. The exponential law for spaces of test functions and diffeomorphism groups. Indagationes Mathematicae, 2015. 6, 32

[62] A. Kriegl and L. D. Nel. A convenient setting for holomorphy. Cahiers Top. Géo. Diff., 26:273-309, 1985. 6

[63] J. Leslie. On a differential structure for the group of diffeomorphisms. Topology, 6:264-271, 1967. 3

[64] T. Marquis and K.-H. Neeb. Half-Lie groups. Transform. Groups, 23(3):801-840, 2018. arXiv:1607.07728. 31

[65] R. Meise and D. Vogt. Introduction to functional analysis, volume 2 of Oxford Graduate Texts in Mathematics. The Clarendon Press, Oxford University Press, New York, 1997. Translated from the German by M. S. Ramanujan and revised by the authors. 15

[66] R. B. Melrose. Differential Analysis on Manifolds with Corners. 1996. http:// www-math.mit.edu/ rbm/book.html 8

[67] M. Micheli, P. W. Michor, and D. Mumford. Sectional curvature in terms of the cometric, with applications to the Riemannian manifolds of landmarks. SIAM J. Imaging Sci., 5(1):394-433, 2012.58

[68] M. Micheli, P. W. Michor, and D. Mumford. Sobolev metrics on diffeomorphism groups and the derived geometry of spaces of submanifolds. Izvestiya: Mathematics, 77(3):541-570, 2013. 35,48 54, 575861

[69] P. W. Michor. Manifolds of differentiable mappings. Shiva Mathematics Series 3,

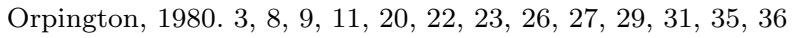

[70] P. W. Michor. Manifolds of smooth maps III: The principal bundle of embeddings of a non compact smooth manifold. Cahiers Top. Geo. Diff., 21:325-337, 1980. MR 82b:58022c. 35

[71] P. W. Michor. Manifolds of smooth mappings IV: Theorem of De Rham. Cahiers Top. Geo. Diff., 24:57-86, 1983. 48

[72] P. W. Michor. A convenient setting for differential geometry and global analysis I, II. Cahiers Topol. Geo. Diff., 25:63-109, 113-178., 1984. 30, 32

[73] P. W. Michor. Topics in differential geometry, volume 93 of Graduate Studies in Mathematics. American Mathematical Society, Providence, RI, 2008. 9, 10,20 
[74] P. W. Michor and D. Mumford. Vanishing geodesic distance on spaces of submanifolds and diffeomorphisms. Doc. Math., 10:217-245, 2005. 49

[75] P. W. Michor and D. Mumford. Riemannian geometries on spaces of plane curves. J. Eur. Math. Soc., 8:1-48, 2006. 36, 49

[76] P. W. Michor and D. Mumford. An overview of the Riemannian metrics on spaces of curves using the Hamiltonian approach. Appl. Comput. Harmon. Anal., 23(1):74113, 2007. 36,48

[77] P. W. Michor and D. Mumford. A zoo of diffeomorphism groups on $\mathbb{R}^{n}$. Ann. Glob. Anal. Geom., 44:529-540, 2013. 7, 32,34

[78] J. Milnor. Remarks on infinite-dimensional Lie groups. In Relativity, groups and topology, II (Les Houches, 1983), pages 1007-1057. North-Holland, Amsterdam, 1984. 28

[79] B. S. Mitjagin. Approximate dimension and bases in nuclear spaces. Uspehi Mat. Nauk, 16(4 (100)):63-132, 1961. 13

[80] D. Mumford and P. W. Michor. On Euler's equation and 'EPDiff'. Journal of Geometric Mechanics, 5:319-344, 2013. 34,54

[81] E. Nelson. Topics in dynamics. I: Flows. Mathematical Notes. Princeton University Press, Princeton, N.J.; University of Tokyo Press, Tokyo, 1969. 18

[82] D. Nenning. ODE-closedness of function spaces and almost analytic extensions of ultradifferentiable functions. $\mathrm{PhD}$ thesis, 2019. Universität Wien. 35

[83] D. Nenning. On time-dependent Besov vector fields and the regularity of their flows, 2019. arXiv:1804.07595. 35

[84] D. N. Nenning and A. Rainer. On groups of Hölder diffeomorphisms and their regularity. Trans. Amer. Math. Soc., 370(8):5761-5794, 2018. 35

[85] D. N. Nenning and A. Rainer. The Trouvé group for spaces of test functions. Rev. R. Acad. Cienc. Exactas Fis. Nat. Ser. A Mat. RACSAM, 113(3):1799-1822, 2019. 3435

[86] H. Omori, Y. Maeda, and A. Yoshioka. On regular Fréchet Lie groups I. Some differential geometric expressions of Fourier integral operators on a Riemannian manifold. Tokyo J. Math., 3:353-390, 1980. 28

[87] H. Omori, Y. Maeda, and A. Yoshioka. On regular Fréchet Lie groups II. Composition rules of Fourier Integral operators on a Riemannian manifold. Tokyo J. Math., 4:221$253,1981.28$

[88] H. Omori, Y. Maeda, and A. Yoshioka. On regular Fréchet Lie groups III. Tokyo J. Math., 4:255-277, 1981.28

[89] H. Omori, Y. Maeda, and A. Yoshioka. On regular Fréchet Lie groups IV. Definitions and fundamental theorems. Tokyo J. Math., 5:365-398, 1982.28

[90] H. Omori, Y. Maeda, and A. Yoshioka. On regular Fréchet Lie groups V. Several basic properties. Tokyo J. Math., 6:39-64, 1983. 28

[91] H. Omori, Y. Maeda, A. Yoshioka, and O. Kobayashi. On regular Fréchet Lie groups VI. Infinite dimensional Lie groups which appear in general relativity. Tokyo $J$. Math., 6:217-246, 1983. 28

[92] R. S. Palais. Foundations of global non-linear analysis. W. A. Benjamin, Inc., New York-Amsterdam, 1968. 3

[93] B. Riemann. Über die Hypothesen, welche der Geometrie zu Grunde liegen. Abhandlungen der Königlichen Gesellschaft der Wissenschaften zu Göttingen, XIII:1-20, 1868. Habilitationsvortrag 10. Juni 1854.2

[94] B. Riemann. On the hypotheses which lie at the bases of geometry. Nature, 8:14-17, 36-37, 1873. translated into English by William Kingdon Clifford. 2

[95] D. M. Roberts and A. Schmeding. Extending Whitney's extension theorem: nonlinear function spaces, 2018. arXiv:1801.04126. 16, 17

[96] L. Schwartz. Théorie des distributions. Hermann, Paris, 1966. Nouvelle édition. 32 33 
[97] R. T. Seeley. Extension of $C^{\infty}$-functions defined in a half space. Proc. AMS, 15:625$626,1964.13$

[98] N. E. Steenrod. A convenient category for topological spaces. Michigan Math. J., 14:133-152, 1967. 4

[99] E. M. Stein. Singular integrals and differentiability properties of functions. Princeton Mathematical Series, No. 30. Princeton University Press, Princeton, N.J., 1970. 13

[100] S. T. Swift. Natural bundles. III. Resolving the singularities in the space of immersed submanifolds. J. Math. Phys., 34(8):3841-3855, 1993. 37

[101] M. Tidten. Fortsetzungen von $C^{\infty}$-Funktionen, welche auf einer abgeschlossenen Menge in $\mathbb{R}^{n}$ definiert sind. Manuscripta Math., 27(3):291-312, 1979. 13,1520

[102] M. Tidten. A geometric characterization for the property $(\underline{D N})$ of $\mathcal{E}(K)$ for arbitrary compact subsets $K$ of $\mathbb{R}$. Arch. Math. (Basel), 77(3):247-252, 2001.20

[103] A. Trouvé. An infinite dimensional group approach for physics based models in pattern recognition. pages 1-35, 1995. http://www.cis.jhu.edu/publications/papers_in database/alain/trouve1995.pdf 34,56

[104] G. Valette. Stokes' formula for stratified forms. Ann. Polon. Math., 114(3):197-206, 2015. 10

[105] D. Vogt. Sequence space representations of spaces of test functions and distributions. In Functional analysis, holomorphy, and approximation theory (Rio de Janeiro, 1979), volume 83 of Lecture Notes in Pure and Appl. Math., pages 405-443. Dekker, New York, 1983. 153233

[106] J. Watts. Diffeologies, Differential Spaces, and Symplectic Geometry. ProQuest LLC, Ann Arbor, MI, 2012. Thesis (Ph.D.)-University of Toronto (Canada). 8

[107] H. Whitney. Analytic extensions of differentiable functions defined in closed sets. Trans. Amer. Math. Soc., 36(1):63-89, 1934. 13

[108] L. Younes. Shapes and diffeomorphisms, volume 171 of Applied Mathematical Sciences. Springer-Verlag, Berlin, 2010. $34,54,56$

Fakultät für Mathematik, Universität Wien, Oskar-Morgenstern-Platz 1, A1090 Wien, Austria.

E-mail address: peter.michor@univie.ac.at. 